\title{
European Resuscitation Council and European Society of Intensive Care Medicine guidelines 2021: post-resuscitation
} care

\author{
Jerry P. Nolan ${ }^{1,2^{*}}$ (1), Claudio Sandroni ${ }^{3,4}$, Bernd W. Böttiger ${ }^{5}$, Alain Cariou ${ }^{6}$, Tobias Cronberg ${ }^{7}$, Hans Friberg $^{8}$, \\ Cornelia Genbrugge ${ }^{9,10}$, Kirstie Haywood ${ }^{11}$, Gisela Lilja ${ }^{12}$, Véronique R. M. Moulaert ${ }^{13}$, Nikolaos Nikolaou ${ }^{14}$, \\ Theresa Mariero Olasveengen ${ }^{15}$, Markus B. Skrifvars ${ }^{16}$, Fabio Taccone ${ }^{17}$ and Jasmeet Soar ${ }^{18}$
}

(C) 2021 European Resuscitation Council

\begin{abstract}
The European Resuscitation Council (ERC) and the European Society of Intensive Care Medicine (ESICM) have collaborated to produce these post-resuscitation care guidelines for adults, which are based on the 2020 International Consensus on Cardiopulmonary Resuscitation Science with Treatment Recommendations. The topics covered include the post-cardiac arrest syndrome, diagnosis of cause of cardiac arrest, control of oxygenation and ventilation, coronary reperfusion, haemodynamic monitoring and management, control of seizures, temperature control, general intensive care management, prognostication, long-term outcome, rehabilitation and organ donation.
\end{abstract}

Keywords: Cardiac arrest, Post resuscitation care, Prognostication, Guidelines

\section{Introduction and scope}

In 2015 the European Resuscitation Council (ERC) and the European Society of Intensive Care Medicine (ESICM) collaborated to produce their first combined post-resuscitation care guidelines, which were co-published in Resuscitation and Intensive Care Medicine [1, 2]. These post-resuscitation care guidelines have been extensively updated for 2020 and incorporate the science that has been published since 2015. The topics covered include the post-cardiac arrest syndrome, control of oxygenation and ventilation, haemodynamic targets, coronary reperfusion, targeted temperature management, control of seizures, prognostication, rehabilitation, and long-term outcome (Fig. 1).

\footnotetext{
*Correspondence: jerry.nolan@nhs.net

1 University of Warwick, Warwick Medical School, Coventry CV4 7AL, UK Full author information is available at the end of the article Jerry P. Nolan and Claudio Sandroni: Joint first authors.
}

\section{Methods}

A comprehensive description of the guideline development process is provided in an electronic supplement.

\section{The international consensus on cardiopulmonary} resuscitation science evidence review process

The International Liaison Committee on Resuscitation (ILCOR, www.ilcor.org) includes representatives from the American Heart Association (AHA), the European Resuscitation Council (ERC), the Heart and Stroke Foundation of Canada (HSFC), the Australian and New Zealand Committee on Resuscitation (ANZCOR), the Resuscitation Council of Southern Africa (RCSA), the Inter-American Heart Foundation (IAHF), and the Resuscitation Council of Asia (RCA). From 2000 to 2015 researchers from the ILCOR member councils evaluated resuscitation science in 5 -yearly cycles. After publication of the 2015 International Consensus on CPR and ECC Science with Treatment Recommendations (2015

\section{自 Springer}




\section{Post-resuscitation care}

Airway and breathing

- Maintain $\mathrm{SpO}_{2} 94$ - 98\%

- Insert advanced airway

- Waveform capnography

- Ventilate lungs to normocapnia

Control temperature

- Constant temperature $32^{\circ} \mathrm{C}-36^{\circ} \mathrm{C}$

- Sedation; control shivering

Circulation

12-lead ECG

- Obtain reliable intravenous access

- Aim for SBP > $100 \mathrm{mmHg}$

- Fluid (crystalloid) - restore normovolaemia

- Intra-arterial blood pressure monitoring

- Consider vasopressor/inotrope to maintain SBP
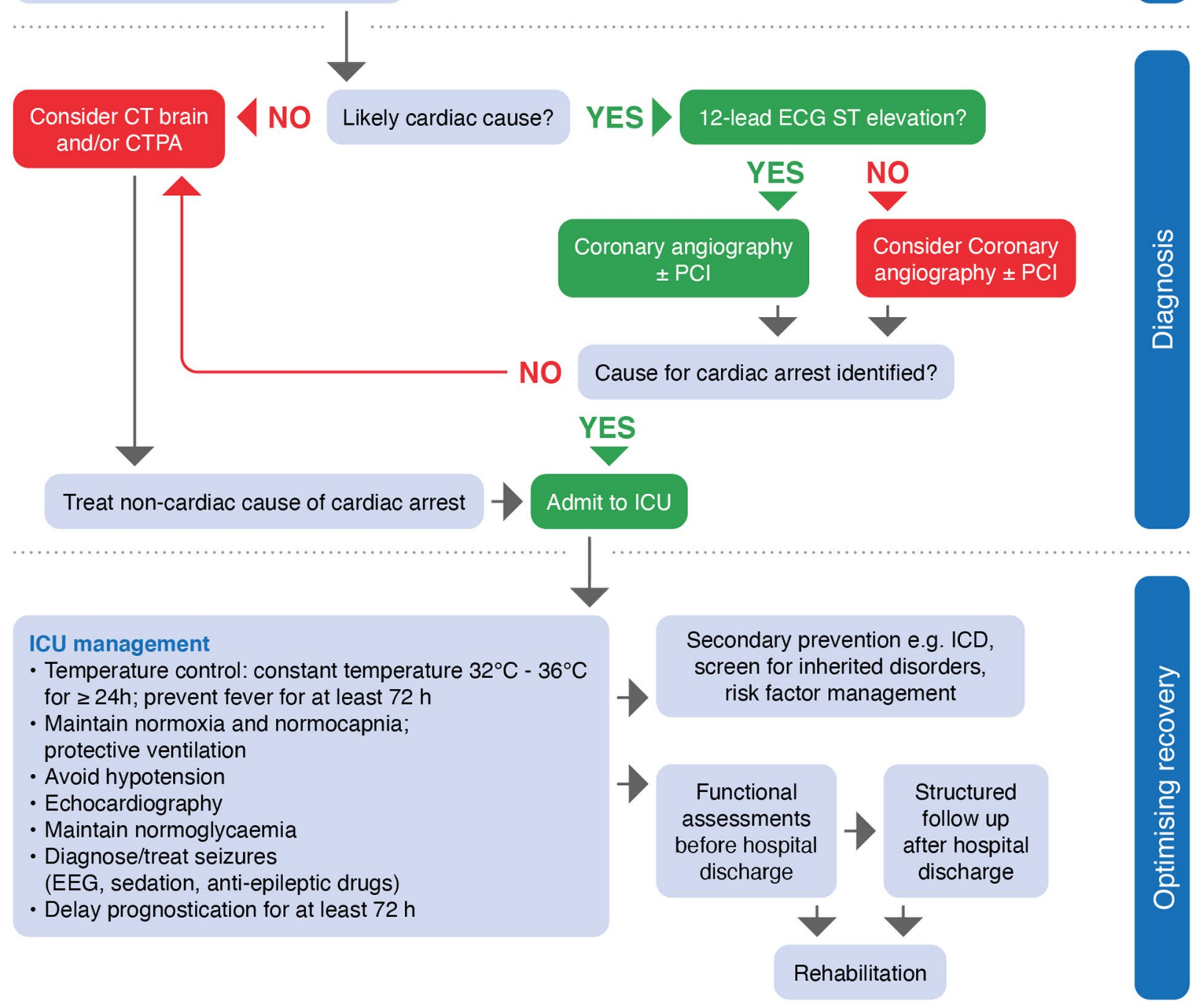

Fig. 1 Post resuscitation care algorithm. SBP systolic blood pressure, PCI percutaneous coronary intervention, CTPA computed tomography pulmonary angiogram, ICU intensive care unit, EEG electroencephalography, ICD implanted cardioverter defibrillator 
CoSTR) [3], ILCOR committed to a continuous evidenceevaluation process, with topics prioritised for review by the task forces and with CoSTR updates published annually [4-6]. For the 2020 CoSTR, the six ILCOR task forces performed three types of evidence evaluation: the systematic review, the scoping review, and the evidence update, which covered 184 topics in total [7]. It was agreed that only systematic reviews [these used Grading of Recommendations Assessment, Development, and Evaluation (GRADE) methodology] could result in new or modified treatment recommendations [8]. The data analysis from each systematic review was presented to the task force, and the task force drafted the summary consensus on science and the treatment recommendations. Each treatment recommendation indicated the strength of the recommendation (recommends=strong, suggests $=$ weak) and the certainty of the evidence. Draft 2020 CoSTRs were posted on the ILCOR website (ilcor. org) for a 2-week comment period after which final wording of science statements and treatment recommendations were completed by the task forces and published in Resuscitation and Circulation as the 2020 Consensus on Science and Treatment Recommendations (CoSTR).

\section{The European Resuscitation Council and European Society for Intensive Care Medicine process for developing post-resuscitation care guidelines}

Fifteen individuals were selected for the ERC-ESICM Post-Resuscitation Care Writing Group based on their expertise, ERC and ESICM representation and diversity (gender, physician and non-physician, and geography (Northern and Southern Europe).

These ERC-ESICM guidelines on post-resuscitation care for adults are based mainly on the Advanced Life Support section of the 2020 CoSTR document and represent consensus among the writing group, which included representatives of the ERC and the ESICM [9]. Where treatment recommendations are provided by ILCOR, these have been adopted by the ERC and ESICM. In the absence of an ILCOR recommendation, ERC-ESICM guidance was based on review and discussion of the evidence by the working group until consensus was achieved. The writing group chairs ensured that everyone on the working group had the opportunity to present and debate their views and ensured that discussions were open and constructive. All discussions took place during eight 2-h Zoom videoconferences that were held between Jan 2020 and November 2020. Consensus was achieved by all 15 writing group members on all the treatment recommendations using an open process.

These guidelines were drafted and agreed by the PostResuscitation Care Writing Group members before posting on the ERC website for public comment between 21
October and 5 November 2020. The opportunity to comment on the guidelines was advertised through social media (Facebook, Twitter) and the ERC network of 33 national resuscitation councils. Nine individuals from four countries made 25 comments. One of these individuals was a lay person. Review of these comments led to eight changes.

\section{Summary of the key changes}

A summary of the main changes from the 2015 ERCESICM Post-resuscitation care guidelines is set out in Table 1.

\section{Concise guidelines for clinical practice}

This section includes only a summary of the main recommendations. The evidence underpinning each recommendation is detailed in the section on 'evidence informing the guidelines'.

\section{Immediate post-resuscitation care}

- Post-resuscitation care is started immediately after sustained ROSC, regardless of location (Fig. 1).

- For out-of-hospital cardiac arrest consider transport to a cardiac arrest centre.

\section{Diagnosis of cause of cardiac arrest}

- If there is clinical (e.g. haemodynamic instability) or ECG evidence of myocardial ischaemia, undertake coronary angiography first. This is followed by CT brain and/or CT pulmonary angiography if coronary angiography fails to identify causative lesions.

- Early identification of a respiratory or neurological cause can be achieved by performing a brain and chest CT-scan at hospital admission, before or after coronary angiography (see coronary reperfusion).

- If there are signs or symptoms pre-arrest suggesting a neurological or respiratory cause (e.g. headache, seizures or neurological deficits, shortness of breath or documented hypoxaemia in patients with known respiratory disease), perform a CT brain and/or a CT pulmonary angiogram.

\section{Airway and breathing}

\section{Airway management after return of spontaneous circulation}

- Airway and ventilation support should continue after return of spontaneous circulation (ROSC) is achieved.

- Patients who have had a brief period of cardiac arrest and an immediate return of normal cerebral function and are breathing normally may not require tracheal intubation but should be given oxygen via a facemask if their arterial blood oxygen saturation is less than $94 \%$. 
Table 1 Summary of changes since the $\mathbf{2 0 1 5}$ Guidelines on Post-resuscitation care

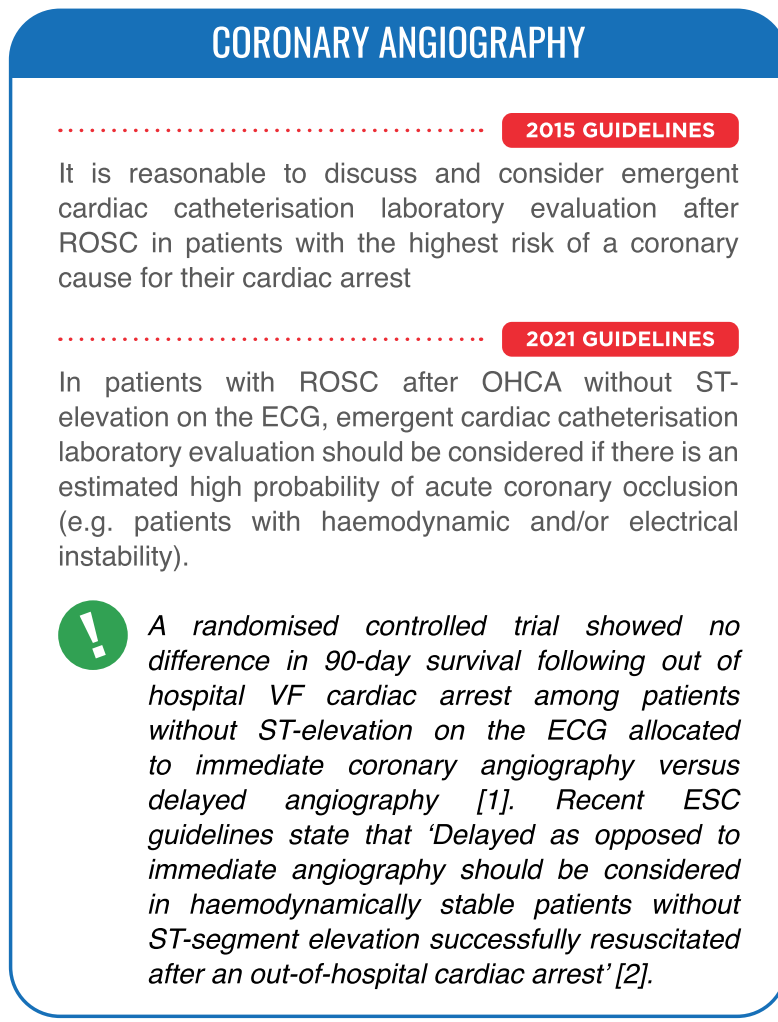

\section{BLOOD PRESSURE TARGET}

Target the mean arterial blood pressure to achieve an adequate urine output $\left(1 \mathrm{ml} \mathrm{kg}^{-1} \mathrm{~h}^{-1}\right)$ and normal or decreasing plasma lactate values, taking into consideration the patient's normal blood pressure, the cause of the arrest and the severity of any myocardial dysfunction.

\section{GUIDELINES}

Avoid hypotension (MAP $<65 \mathrm{mmHg}$ ). Target MAP to achieve adequate urine output $\left(>0.5 \mathrm{~mL} \mathrm{~kg}^{-1} \mathrm{~h}^{-1}\right)$ and normal or decreasing lactate.

Several studies show that hypotension (MAP $<65 \mathrm{mmHg}$ ) is consistently associated with poor outcome. Although we have stated a threshold value for blood pressure, optimal MAP targets are likely to need to be individualised.
TREATMENT OF SEIZURES

\section{GUIDELINES}

Treat [seizures] with sodium valproate, levetiracetam, phenytoin, benzodiazepines, propofol, or a barbiturate.

\section{GUIDELINES}

To treat seizures after cardiac arrest, we suggest levetiracetam or sodium valproate as first-line antiepileptic drugs in addition to sedative drugs.

In a recently reported trial, valproate, levetiracetam and fosphenytoin were equally effective in terminating convulsive status epilepticus but fosphenytoin caused more episodes of hypotension [3].

\section{TEMPERATURE GONTROL}

\section{GUIDELINES}

- Maintain a constant, target temperature between $32^{\circ} \mathrm{C}$ and $36^{\circ} \mathrm{C}$ for those patients in whom temperature control is used (strong recommendation, moderatequality evidence).

- Whether certain subpopulations of cardiac arrest patients may benefit from lower $\left(32-34^{\circ} \mathrm{C}\right)$ or higher $\left(36^{\circ} \mathrm{C}\right)$ temperatures remains unknown, and further research may help elucidate this.

- TTM is recommended for adults after OHCA with an initial shockable rhythm who remain unresponsive after ROSC (strong recommendation, low-quality evidence).

- TTM is suggested for adults after OHCA with an initial non-shockable rhythm who remain unresponsive after ROSC (weak recommendation, very low-quality evidence).

- TTM is suggested for adults after IHCA with any initial rhythm who remain unresponsive after ROSC (weak recommendation, very low-quality evidence).

- If targeted temperature management is used, it is suggested that the duration is at least $24 \mathrm{~h}$ (weak recommendation, very low-quality evidence).

\section{GUIDELINES}

- We recommend TTM for adults after either OHCA or IHCA (with any initial rhythm) who remain unresponsive after ROSC.

- Maintain a target temperature at a constant value between $32^{\circ} \mathrm{C}$ and $36^{\circ} \mathrm{C}$ for at least $24 \mathrm{~h}$.

- Avoid fever $\left(>37.7^{\circ} \mathrm{C}\right)$ for at least $72 \mathrm{~h}$ after ROSC in patients who remain in coma. 
Table 1 (continued)

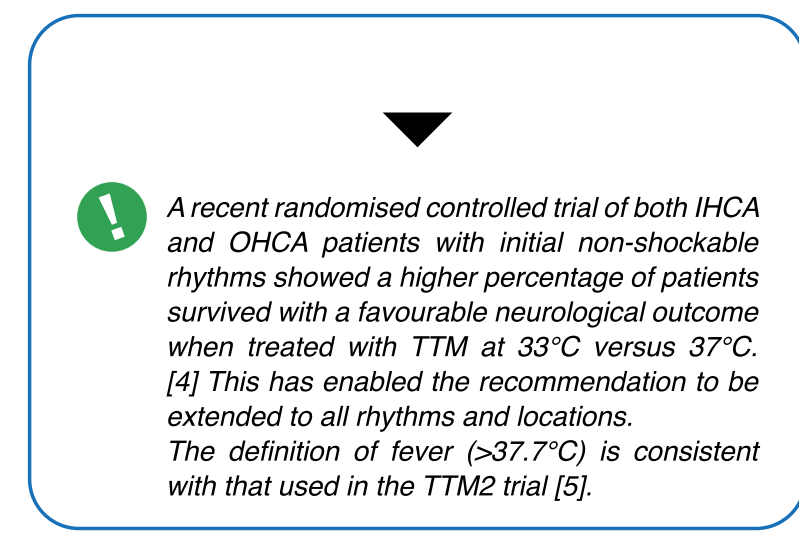

\section{GENERAL INTENSIVE CARE MANAGEMENT}

Short-acting drugs (e.g., propofol, alfentanil, remifentanil) will enable more reliable and earlier neurological assessment and prognostication.

Following ROSC maintain the blood glucose at $\leq 10$ $\mathrm{mmol} \mathrm{I}^{-1}\left(180 \mathrm{mg} \mathrm{dl}^{-1}\right)$ and avoid hypoglycaemia.

\section{GUIDELINES}

- Use short acting sedatives and opioids.

- Avoid using a neuromuscular blocking drug routinely in patients undergoing TTM, but it may be considered in case of severe shivering during TTM.

- Provide stress ulcer prophylaxis routinely in cardiac arrest patients.

- Provide deep venous thrombosis prophylaxis.

- Target a blood glucose of 7.8-10 mmol L-1 (140-180 $\mathrm{mg} \mathrm{dL}^{-1}$ ) using an infusion of insulin if required; avoid hypoglycaemia $\left(<4.0 \mathrm{mmol} \mathrm{L}^{-1}\left(<70 \mathrm{mg} \mathrm{dL}^{-1}\right)\right.$.

- Start enteral feeding at low rates (trophic feeding) during TTM and increase after rewarming if indicated. If TTM of $36^{\circ} \mathrm{C}$ is used as the target temperature, trophic gastric feeding rates may be increased early during TTM.

- We do not recommend using prophylactic antibiotics routinely.

The 2015 guidelines included very few statements on general intensive care management. For 2020 we have several best practice statements based mainly on data extrapolated from other critically ill patient groups.

\section{PROGNOSTICATION}

The prognostication strategy algorithm is applicable to all patients who remain comatose with an absent or extensor motor response to pain at $\geq 72 \mathrm{~h}$ from ROSC. Results of earlier prognostic tests are also considered at this time point.

One or both of the following indicate that a poor outcome is very likely (FPR $<5 \%$, narrow $95 \% \mathrm{Cls}$ ):

- No pupillary and corneal reflexes

- Bilaterally absent N20 SSEP wave

Two or more of the following indicate that a poor outcome is likely:

- Status myoclonus $\leq 48 \mathrm{~h}$ after ROSC

- High NSE levels

- Unreactive burst-suppression or status epilepticus on EEG

- Diffuse anoxic injury on brain CT/MRI

In a comatose patient with $\mathrm{M} \leq 3$ at $\geq 72 \mathrm{~h}$ from ROSC, in the absence of confounders, poor outcome is likely when two or more of the following predictors are present:

- no pupillary and corneal reflexes at $\geq 72 \mathrm{~h}$,

- bilaterally absent N20 SSEP wave at $\geq 24 \mathrm{~h}$,

- highly malignant EEG (suppressed background or burst-suppression) at $>24 \mathrm{~h}$,

- NSE $>60 \mu \mathrm{g} \mathrm{L}^{-1}$ at $48 \mathrm{~h}$ and/or $72 \mathrm{~h}$,

- status myoclonus $\leq 72 \mathrm{~h}$,

- or a diffuse and extensive anoxic injury on brain CT/ MRI.

There has been a very large amount of data published on prognostication since the 2015 guidelines. A recent systematic review identified 94 studies that included over 30,000 patients, all published since January 2013 [6].

The two-stage prognostication algorithm in the 2015 guidelines has been simplified so that a poor outcome is considered likely when two or more of the listed predictors are present. The algorithm is valid for comatose patients with a Glasgow Motor Score $\leq 3$ (compared with $\leq 2$ in the 2015 version). A threshold value for NSE is now stated. The EEG patterns suppression and burst-suppression are the most consistent predictors of poor neurological outcome. Conversely, absence of EEG reactivity has been only inconsistently associated with poor neurological outcome in recent studies.

We suggest using the 2021 ACNS terminology when assessing these patterns for prognostication, to ensure an unequivocal identification. 
Table 1 (continued)

REHABILITATION
Follow-up care should be organised systematically and
can be provided by a physician or specialised nurse. It
includes at least the following aspects:
. Screening for cognitive impairments
. Screening for emotional problems
. Provision of information
...................................... 2021 GUIDELINEs
. Perform functional assessments of physical and
non-physical impairments before discharge from the
hospital to identify early rehabilitation needs and refer
to rehabilitation if necessary.
- Organise follow-up for all cardiac arrest survivors
within 3 months after hospital discharge, including:
1. Screening for cognitive problems.
2. Screening for emotional problems and fatigue.
3. Providing information and support for survivors and
family members.
The authorship of the 2021 guidelines now
includes 3 individuals with expertise on long-
term outcomes and rehabilitation after cardiac
arrest compared with one author in 2015. The
2021 guidelines include greater emphasis on
functional assessments of physical and non-
physical impairments before discharge and long-
term follow up and rehabilitation. There is greater
recognition of the importance of survivorship
after cardiac arrest. The recommendations in
this section are all best practice statements.

CARDIAC ARREST CENTRES
No specific recommendation.
An expert consensus paper published by
several European organisations including the
Association of Acute Cardiovascular Care
(ACVA) of the European Society of Cardiology
(ESC), the ERC and the ESICM, states that
the minimum requirements for a cardiac
arrest centre are $24 / 7$ availability of an on-site
coronary angiography laboratory, an emergency
department, an ICU, imaging facilities, such
as echocardiography, CT, and MRI [7]. Based
on evidence from a systematic review, ILCOR
suggests that wherever possible, adult patients
with non-traumatic OHCA cardiac arrest should
be cared for in cardiac arrest centres [8].

ACNS American Clinical Neurophysiology Society

CT computed tomography

ESC European Society of Cariology

EEG electroencephalogram

FPR false positive rate

ILCOR International Liaison Committee on Resuscitation

IHCA in-hospital cardiac arrest

MAP mean arterial pressure
MRI magnetic resonance imaging

NSE neuron specific enolase

OHCA out-of-hospital cardiac arrest

ROSC return of spontaneous circulation

SSEP somatosensory evoked potential

TTM targeted temperature management

VF ventricular fibrillation

REFERENCES

1. Lemkes JS, Janssens GN, van der Hoeven NW, et al. (2019) Coronary Angiography after Cardiac Arrest without ST-Segment Elevation. N Engl J Med 380: $1397-1407$

2. Collet JP, Thiele H, Barbato E, et al. (2020) 2020 ESC Guidelines for the management of acute coronary syndromes in patients presenting without persistent ST-segment elevation. Eur Heart J

3. Kapur J, Elm J, Chamberlain JM, et al. (2019) Randomized Trial of Three Anticonvulsant Medications for Status Epilepticus. N Engl J Med 381: 2103-2113

3. Kapur J, Elm J, Chamberlain JM, et al. (2019) Randomized Trial of Three Anticonvulsant Medications for Status Epilepticus. N Engl J Med 381: $2103-2113$

5. Dankiewicz J, Cronberg T, Lilja G, et al. (2019) Targeted hypothermia versus targeted Normothermia after out-of-hospital cardiac arrest (TTM2): A randomized clinical trial-Rationale and design. Am Heart J 217: 23-31

6. Sandroni C, D'Arrigo S, Cacciola S, et al. (2020) Prediction of poor neurological outcome in comatose survivors of cardiac arrest: a systematic review. Intensive Care Med 46: $1803-1851$

7. Sinning C, Ahrens I, Cariou A, et al. (2020) The cardiac arrest centre for the treatment of sudden cardiac arrest due to presumed cardiac cause - aims, function and structure: Position paper of the Association for Acute CardioVascular Care of the European Society of Cardiology (AVCV), European Association of Percutaneous Coronary Interventions (EAPCI), European Heart Rhythm Association (EHRA), European Resuscitation Council (ERC), European Society for Emergency Medicine (EUSEM) and European Society of Intensive Care Medicine (ESICM). Eur Heart J Acute Cardiovasc Care 9: S193-S202

8. Yeung J, Matsuyama T, Bray J, Reynolds J, Skrifvars MB. (2019) Does care at a cardiac arrest centre improve outcome after out-of-hospital cardiac arrest? - A systematic review. Resuscitation 137: 102-115 
- Patients who remain comatose following ROSC, or who have another clinical indication for sedation and mechanical ventilation, should have their trachea intubated if this has not been done already during CPR.

- Tracheal intubation should be performed only by experienced operators who have a high success rate.

- Correct placement of the tracheal tube must be confirmed with waveform capnography.

- In the absence of personnel experienced in tracheal intubation, it is reasonable to insert a supraglottic airway (SGA) or maintain the airway with basic techniques until skilled intubators are available.

\section{Control of oxygenation}

- After ROSC, use 100\% (or maximum available) inspired oxygen until the arterial oxygen saturation or the partial pressure of arterial oxygen can be measured reliably.

- After ROSC, once $\mathrm{SpO}_{2}$ can be measured reliably or arterial blood gas values are obtained, titrate the inspired oxygen to achieve an arterial oxygen satura- tion of 94-98\% or arterial partial pressure of oxygen $\left(\mathrm{PaO}_{2}\right)$ of $10-13 \mathrm{kPa}$ or $75-100 \mathrm{mmHg}$ (Fig. 2).

- Avoid hypoxaemia $\left(\mathrm{PaO}_{2}<8 \mathrm{kPa}\right.$ or $\left.60 \mathrm{mmHg}\right)$ following ROSC.

- Avoid hyperoxaemia following ROSC.

\section{Control of ventilation}

- Obtain an arterial blood gas and use end tidal $\mathrm{CO}_{2}$ in mechanically ventilated patients.

- In patients requiring mechanical ventilation after ROSC, adjust ventilation to target a normal arterial partial pressure of carbon dioxide $\left(\mathrm{PaCO}_{2}\right)$, i.e. 4.5$6.0 \mathrm{kPa}$ or $35-45 \mathrm{mmHg}$.

- In patients treated with targeted temperature management (TTM) monitor $\mathrm{PaCO}_{2}$ frequently as hypocapnia may occur.

- During TTM and lower temperatures use consistently either a temperature or non-temperature corrected approach for measuring blood gas values.

- Use a lung protective ventilation strategy aiming for a tidal volume of 6-8 $\mathrm{mL} \mathrm{kg}^{-1}$ ideal body weight.

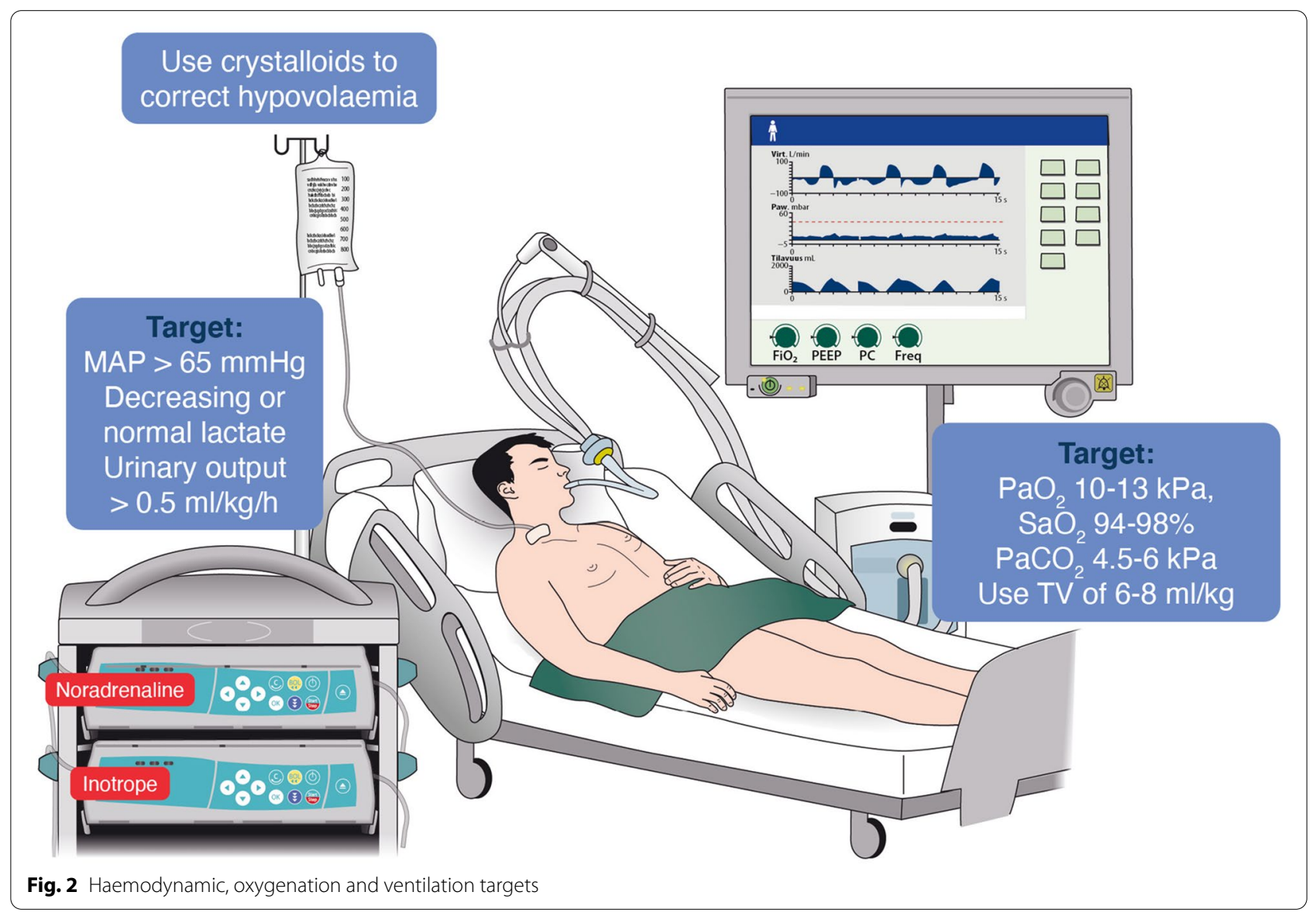




\section{Circulation}

\section{Coronary reperfusion}

- Emergent cardiac catheterisation laboratory evaluation (and immediate PCI if required) should be performed in adult patients with ROSC after cardiac arrest of suspected cardiac origin with ST-elevation on the ECG.

- In patients with ROSC after out-of-hospital cardiac arrest (OHCA) without ST-elevation on the ECG, emergent cardiac catheterisation laboratory evaluation should be considered if there is an estimated high probability of acute coronary occlusion (e.g. patients with haemodynamic and/or electrical instability).

\section{Haemodynamic monitoring and management}

- All patients should be monitored with an arterial line for continuous blood pressure measurements, and it is reasonable to monitor cardiac output in haemodynamically unstable patients.

- Perform early (as soon as possible) echocardiography in all patients to detect any underlying cardiac pathology and quantify the degree of myocardial dysfunction.

- Avoid hypotension (<65 mmHg). Target mean arterial pressure (MAP) to achieve adequate urine output (>0.5 mL kg-1 $\mathrm{h}^{-1}$ ) and normal or decreasing lactate (Fig. 2).

- During TTM at $33{ }^{\circ} \mathrm{C}$, bradycardia may be left untreated if blood pressure, lactate, $\mathrm{ScvO}_{2}$ or $\mathrm{SvO}_{2}$ is adequate. If not, consider increasing the target temperature, but to no higher than $36^{\circ} \mathrm{C}$.

- Maintain perfusion with fluids, noradrenaline and/ or dobutamine, depending on individual patient need for intravascular volume, vasoconstriction or inotropy.

- Do not give steroids routinely after cardiac arrest.

- Avoid hypokalaemia, which is associated with ventricular arrhythmias.

- Consider mechanical circulatory support (such as intra-aortic balloon pump, left-ventricular assist device or arterio-venous extra corporal membrane oxygenation) for persisting cardiogenic shock from left ventricular failure if treatment with fluid resuscitation, inotropes and vasoactive drugs is insufficient. Left-ventricular assist devices or arteriovenous extra corporal membrane oxygenation should also be considered in haemodynamically unstable patients with acute coronary syndromes (ACS) and recurrent ventricular tachycardia (VT) or ventricular fibrillation (VF) despite optimal therapy.

\section{Disability (optimising neurological recovery) Control of seizures}

- We recommend using electroencephalography (EEG) to diagnose electrographic seizures in patients with clinical convulsions and to monitor treatment effects.

- To treat seizures after cardiac arrest, we suggest levetiracetam or sodium valproate as first-line antiepileptic drugs in addition to sedative drugs.

- We suggest that routine seizure prophylaxis is not used in post-cardiac arrest patients.

\section{Temperature control}

- We recommend targeted temperature management (TTM) for adults after either OHCA or in-hospital cardiac arrest (IHCA) (with any initial rhythm) who remain unresponsive after ROSC.

- Maintain a target temperature at a constant value between 32 and $36^{\circ} \mathrm{C}$ for at least $24 \mathrm{~h}$.

- Avoid fever $\left(>37.7^{\circ} \mathrm{C}\right)$ for at least $72 \mathrm{~h}$ after ROSC in patients who remain in coma.

- Do not use pre-hospital intravenous cold fluids to initiate hypothermia.

\section{General intensive care management}

- Use short acting sedatives and opioids.

- Avoid using a neuromuscular blocking drug routinely in patients undergoing TTM, but it may be considered in case of severe shivering during TTM.

- Provide stress ulcer prophylaxis routinely in cardiac arrest patients.

- Provide deep venous thrombosis prophylaxis.

- Target a blood glucose of 7.8-10 mmol L-1 (140$180 \mathrm{mg} \mathrm{dL}^{-1}$ ) using an infusion of insulin if required; avoid hypoglycaemia $\left(<4.0 \mathrm{mmol} \mathrm{L}^{-1}\left(<70 \mathrm{mg} \mathrm{dL}^{-1}\right)\right.$

- Start enteral feeding at low rates (trophic feeding) during TTM and increase after rewarming if indicated. If TTM of $36^{\circ} \mathrm{C}$ is used as the target temperature, gastric feeding rates may be increased early during TTM.

- We do not recommend using prophylactic antibiotics routinely.

\section{Prognostication \\ General guidelines}

- In patients who are comatose after resuscitation from cardiac arrest, neurological prognostication should be performed using clinical examination, electrophysiology, biomarkers and imaging, to both inform patient's relatives and to help clinicians to target treatments based on the patient's chances of achieving a neurologically meaningful recovery (Fig. 3). 


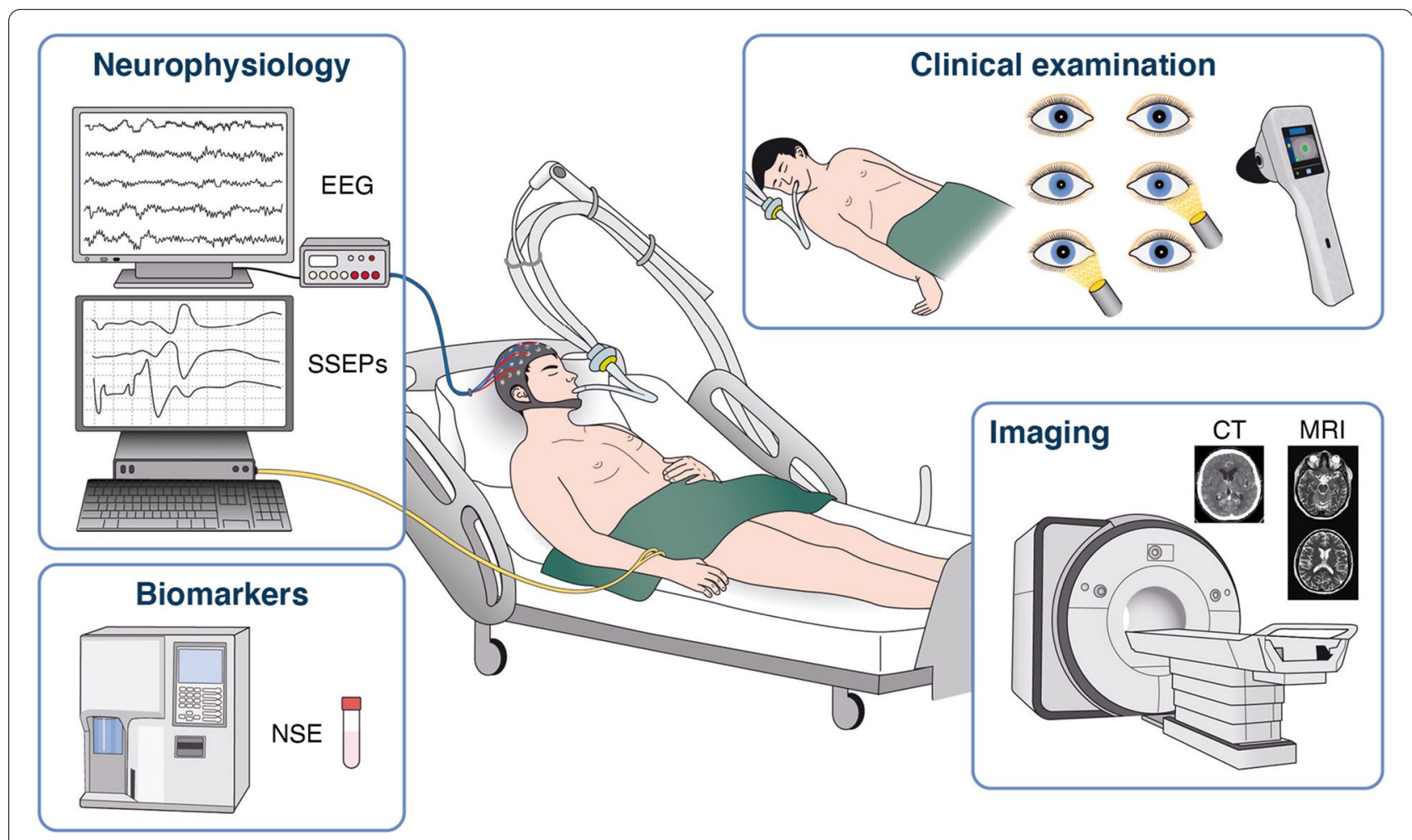

Fig. 3 Prognostication modes. EEG electroencephalography, NSE neuron specific enolase, SSEP somatosensory evoked potential

- No single predictor is $100 \%$ accurate. Therefore, a multimodal neuroprognostication strategy is recommended.

- When predicting poor neurological outcome, a high specificity and precision are desirable, to avoid falsely pessimistic predictions.

- The clinical neurological examination is central to prognostication. To avoid falsely pessimistic predictions, clinicians should avoid potential confounding from sedatives and other drugs that may confound the results of the tests.

- When patients are treated with TTM, daily clinical examination is advocated but final prognostic assessment should be undertaken only after rewarming.

- Clinicians must be aware of the risk of a self-fulfilling prophecy bias, occurring when the results of an index test predicting poor outcome is used for treatment decisions, especially regarding life-sustaining therapies.

- Index tests for neurological prognostication are aimed at assessing the severity of hypoxic-ischaemic brain injury. The neurological prognosis is one of several aspects to consider in discussions around an individual's potential for recovery.

\section{Multimodal prognostication}

- Start the prognostication assessment with an accurate clinical examination, to be performed only after major confounders (e.g. residual sedation, hypothermia) have been excluded (Fig. 4)

- In a comatose patient with $M \leq 3$ at $\geq 72 \mathrm{~h}$ from ROSC, in the absence of confounders, poor outcome is likely when two or more of the following predictors are present: no pupillary and corneal reflexes at $\geq 72 \mathrm{~h}$, bilaterally absent N20 SSEP wave at $\geq 24 \mathrm{~h}$, highly malignant EEG at $>24 \mathrm{~h}$, neuron-specific enolase (NSE) $>60 \mu \mathrm{g} \mathrm{L}^{-1}$ at $48 \mathrm{~h}$ and/or $72 \mathrm{~h}$, status myoclonus $\leq 72 \mathrm{~h}$, or a diffuse and extensive anoxic injury on brain CT/MRI. Most of these signs can be recorded before $72 \mathrm{~h}$ from ROSC; however, their results will be evaluated only at the time of clinical prognostic assessment.

\section{Clinical examination}

- Clinical examination is prone to interference from sedatives, opioids or muscle relaxants. A potential confounding from residual sedation should always be considered and excluded.

- A Glasgow Motor Score of $\leq 3$ (abnormal flexion or worse in response to pain) at $72 \mathrm{~h}$ or later after ROSC 


\section{Resuscitation from cardiac arrest}

\section{Targeted temperature management and rewarming}

Unconscious patient, $\mathrm{M} \leq 3$ at $\geq 72 \mathrm{~h}$ without confounders ${ }^{(1)}$

\section{YES}

\section{At least TWO of:}

- No pupillary ${ }^{(2)}$ and corneal reflexes at $\geq 72 h$

- Bilaterally absent N20 SSEP wave

- Highly malignant ${ }^{(3)}$ EEG at $>24 \mathrm{~h}$

- NSE $>60 \mu \mathrm{g} / \mathrm{L}^{(4)}$ at $48 \mathrm{~h}$ and/or 72h

- Status myoclonus(5) $\leq 72 \mathrm{~h}$

- Diffuse and extensive anoxic injury on brain CT/MRI

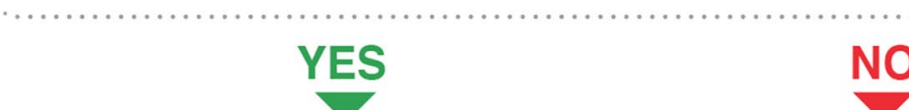

\section{Poor outcome likely(}

\section{Observe and re-evaluate}

Fig. 4 Prognostication strategy algorithm. EEG electroencephalography, NSE neuron specific enolase, SSEP somatosensory evoked potential, ROSC return of spontaneous circulation. 1. Major confounders may include sedation, neuromuscular blockade, hypothermia, severe hypotension, hypoglycaemia, sepsis, and metabolic and respiratory derangements. 2. Use an automated pupillometer, when available, to assess pupillary light reflex. 3 . Suppressed background \pm periodic discharges or burst-suppression, according to ACNS. 4. Increasing NSE values between $24 \mathrm{~h}-48 \mathrm{~h}$ or $24 / 48 \mathrm{~h}$ and $72 \mathrm{~h}$ further confirm a likely poor outcome. 5. Defined as a continuous and generalised myoclonus persisting for 30 min or more. ${ }^{*}$ Caution in case of discordant signs indicating a potentially good outcome (see text for details)

may identify patients in whom neurological prognostication may be needed.

- In patients who remain comatose at $72 \mathrm{~h}$ or later after ROSC the following tests may predict a poor neurological outcome:

- The bilateral absence of the standard pupillary light reflex.

- Quantitative pupillometry

- The bilateral absence of corneal reflex

- The presence of myoclonus within $96 \mathrm{~h}$ and, in particular, status myoclonus within $72 \mathrm{~h}$

- We also suggest recording the EEG in the presence of myoclonic jerks in order to detect any associated epileptiform activity or to identify EEG signs, such as background reactivity or continuity, suggesting a potential for neurological recovery.

\section{Neurophysiology}

- Perform an EEG in patients who are unconscious after the arrest.

- Highly malignant EEG-patterns include suppressed background with or without periodic discharges and burst-suppression. We suggest using these EEG-patterns after the end of TTM and after sedation has been cleared as indicators of a poor prognosis.

- The presence of unequivocal seizures on EEG during the first $72 \mathrm{~h}$ after ROSC is an indicator of a poor prognosis. 


\section{BEFORE HOSPITAL DISCHARGE}

Perform functional assessments

of physical and non-physical impairments

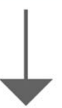

Refer to rehabilitation if necessary

AT FOLLOW UP

Within 3 months from hospital discharge

\section{Perform screeening for cognitive problems}

Perform screening for emotional problems and fatigue
Provide information and support to the survivor and their family

\section{Consider referral to further specialised care if indicated}

Fig. 5 Recommendations for in-hospital functional assessments, follow-up and rehabilitation after cardiac arrest

- Absence of background reactivity on EEG is an indicator of poor prognosis after cardiac arrest.

- Bilateral absence of somatosensory evoked cortical N20-potentials is an indicator of poor prognosis after cardiac arrest.

- Always consider the results of EEG and somatosensory evoked potentials (SSEP) in the context of clinical examination findings and other tests. Always consider using a neuromuscular blocking drug when performing SSEP.

\section{Biomarkers}

- Use serial measurements of NSE in combination with other methods to predict outcome after cardiac arrest. Increasing values between 24 and $48 \mathrm{~h}$ or $72 \mathrm{~h}$ in combination with high values at 48 and $72 \mathrm{~h}$ indicates a poor prognosis.

\section{Imaging}

- Use brain imaging studies for predicting poor neurological outcome after cardiac arrest in combination with other predictors, in centres where specific experience in these studies is available.

- Use presence of generalised brain oedema, manifested by a marked reduction of the grey matter/ white matter ratio on brain $\mathrm{CT}$, or extensive diffusion restriction on brain MRI to predict poor neurological outcome after cardiac arrest.

- Always consider findings from imaging in combination with other methods for neurological prognostication.

\section{Withdrawal of life-sustaining therapy}

- Separate discussions around withdrawal of life-sustaining therapy (WLST) and the assessment of prognosis for neurological recovery; WLST decisions should consider aspects other than brain injury such as age, co-morbidity, general organ function and the patients' preferences. 
Unresponsive patient after ROSC
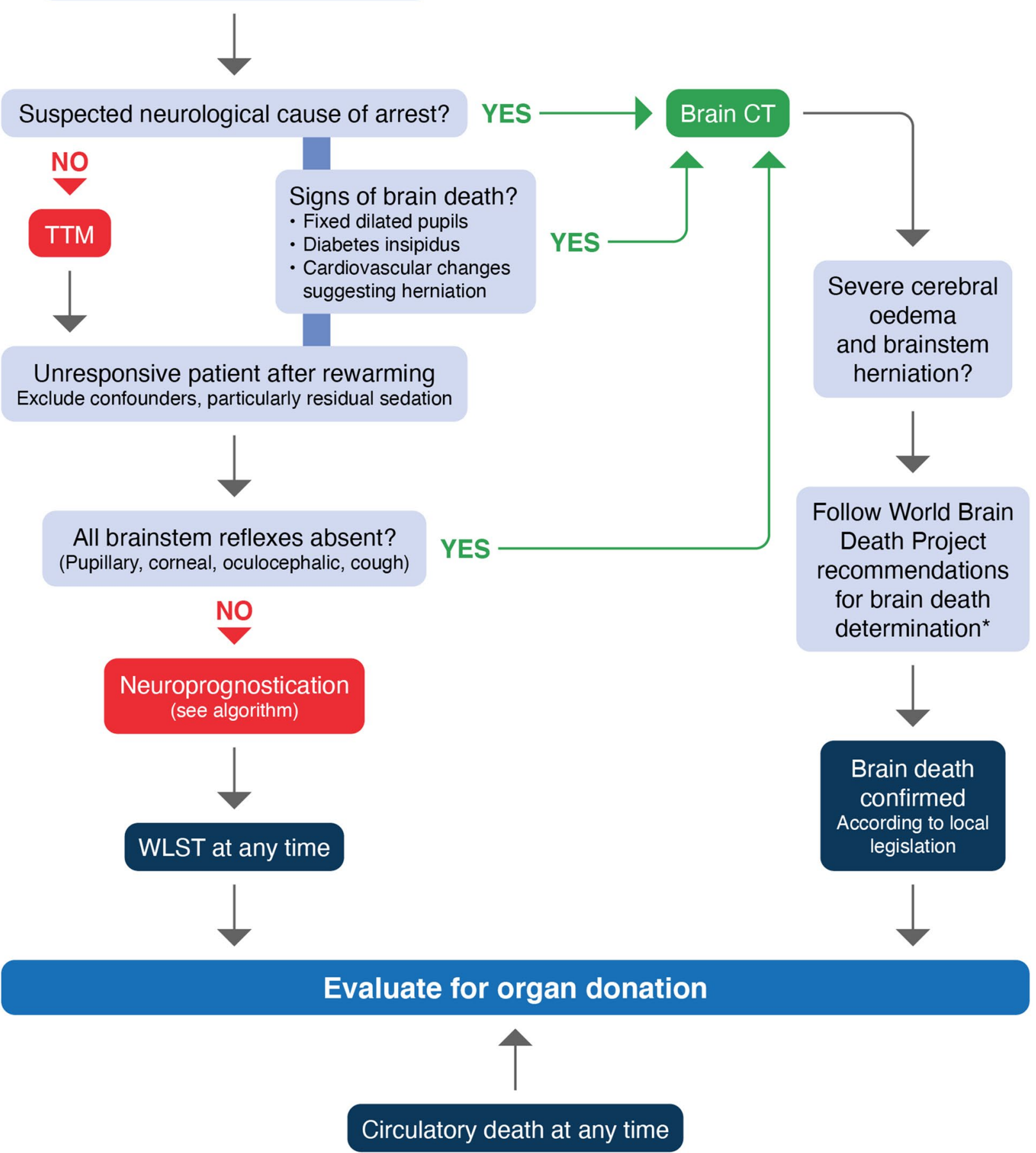

Fig. 6 Organ donation after cardiac arrest algorithm. *Includes a 24-h observation period after rewarming to $36{ }^{\circ} \mathrm{C}$ before clinical testing for brain death/death by neurological criteria [406]. WLST withdrawal of life sustaining treatment Adapted from [286]

- Allocate sufficient time for communication around the level-of-treatment decision within the team and with the relatives.

\section{Long-term outcome after cardiac arrest}

- Perform functional assessments of physical and nonphysical impairments before discharge from the hos- 
pital to identify early rehabilitation needs and refer to rehabilitation if necessary (Fig. 5).

- Organise follow-up for all cardiac arrest survivors within 3 months after hospital discharge, including the following:

1. Screening for cognitive problems.

2. Screening for emotional problems and fatigue.

3. Providing information and support for survivors and family members.

\section{Organ donation}

- All decisions concerning organ donation must follow local legal and ethical requirements.

- Organ donation should be considered in those who have achieved ROSC and who fulfil neurological criteria for death (Fig. 6).

- In comatose ventilated patients who do not fulfil neurological criteria for death, if a decision to start end-of-life care and withdrawal of life support is made, organ donation should be considered for when circulatory arrest occurs.

\section{Cardiac arrest centres}

- Adult patients with non-traumatic OHCA should be considered for transport to a cardiac arrest centre according to local protocol.

\section{Evidence informing the guidelines}

\section{Post-cardiac arrest syndrome}

The post-cardiac arrest syndrome comprises post-cardiac arrest hypoxic-ischaemic brain injury, post-cardiac arrest myocardial dysfunction, the systemic ischaemia/ reperfusion response, and the persistent precipitating pathology [18-21]. The severity of this syndrome will vary with the duration and cause of cardiac arrest. It may not occur at all if the cardiac arrest is brief. Among patients surviving to intensive care unit (ICU) admission but subsequently dying in-hospital, withdrawal of treatment following prognostication of poor neurological outcome is the cause of death in approximately two-thirds after OHCA and approximately 25\% after in-hospital cardiac arrest [22-26]. Cardiovascular failure accounts for most deaths in the first 3 days, while, in many countries, WLST based on a prognostication of severe hypoxic-ischaemic brain injury accounts for most of the later deaths [23, 26, 27]. Post-cardiac arrest hypoxic-ischaemic brain injury is associated with hypotension, hypoxaemia, hyperoxaemia, pyrexia, hypoglycaemia, hyperglycaemia and seizures. Significant myocardial dysfunction is common after cardiac arrest but typically starts to recover by $2-3$ days, although full recovery may take significantly longer [28-33]. The whole-body ischaemia/reperfusion of cardiac arrest, CPR and ROSC activates immune and coagulation pathways contributing to multiple organ failure and increasing the risk of infection [34-43]. Thus, the post-cardiac arrest syndrome has many features in common with sepsis, including intravascular volume depletion, vasodilation, endothelial injury and abnormalities of the microcirculation [44-53].

\section{Diagnosis of cause of cardiac arrest}

These guidelines are informed by expert consensus.

Cardiac causes of OHCA have been studied extensively in the last few decades; conversely, little is known about non-cardiac causes. Early identification of a respiratory or neurological cause would enable transfer of the patient to a specialised ICU for optimal care. Improved knowledge of prognosis also enables discussion about the appropriateness of specific therapies, including TTM. Several case series showed that this strategy enables diagnosis of non-cardiac causes of arrest in a substantial proportion of patients $[54,55]$. There is considerable regional variation in the incidence of sub-arachnoid haemorrhage as a cause of cardiac arrest among those with sustained ROSC at hospital admission. Published case series report $16.2 \%$ in Japan [56], 11.4\% in Korea [57] and 7\% in France [58]. In those with cardiac arrest associated with trauma or haemorrhage a whole-body CT scan is likely indicated $[9,59,60]$.

\section{Airway and breathing Airway management after return of spontaneous circulation} These guidelines are informed by expert consensus.

Patients can have their trachea intubated before, during or following cardiac arrest depending on the setting or particular circumstances [61]. Following most cardiac arrests tracheal intubation will occur during CPR or if the patient remains comatose after ROSC [62].

Tracheal intubation following ROSC in comatose patients will facilitate post-resuscitation care that includes controlled oxygenation and ventilation, protection of the lungs from aspiration of stomach contents, control of seizures and TTM-see below for further details.

Post ROSC patients are haemodynamically unstable and, depending on their level of consciousness, may require drug assisted tracheal intubation. The same level of care should be provided as for any other critically ill patient in terms of skills of the provider, monitoring and choice of drugs $[63,64]$. There are no recommendations for a specific drug combination [65], but use of a low 
dose of a sedative, an analgesic and a rapid onset neuromuscular blocking drug is probably optimal.

\section{Control of oxygenation}

These guidelines are informed by the ILCOR systematic review on oxygenation and ventilation targets after cardiac arrest, which identified seven RCTs and 36 observational studies [66], and CoSTR [9]. The ILCOR treatment recommendations in relation to oxygenation are as follows:

- We suggest the use of $100 \%$ inspired oxygen until the arterial oxygen saturation or the partial pressure of arterial oxygen can be measured reliably in adults with ROSC after cardiac arrest in any setting (weak recommendation, very-low certainty evidence).

- We recommend avoiding hypoxaemia in adults with ROSC after cardiac arrest in any setting (strong recommendation, very-low certainty evidence).

- We suggest avoiding hyperoxaemia in adults with ROSC after cardiac arrest in any setting (weak recommendation, low-certainty evidence).

From a pathophysiological perspective, post cardiac arrest patients are at risk of developing hypoxic-ischaemic brain injury and accompanying organ dysfunction $[9,21,67,68]$. The role of blood oxygen values in the disease process is poorly understood [69]. Studies show that cerebral ischaemia in post cardiac arrest patients is associated with poor outcome [70]. Administering more oxygen can increase brain oxygenation [71]. On the other hand, higher oxygen values would logically cause an increase in harmful oxygen free radicals [72]. It is also likely that the effect of oxygen values varies between different organs such as the heart and brain.

Numerous experimental studies have assessed the impact of hyperoxaemia on neurological injury with mixed findings [73]. Six randomised controlled trials (RCTs) have compared different oxygenation targets for varying durations immediately and up to $48 \mathrm{~h}$ after ROSC [74-79]. A sub-group analysis of a large RCT targeting an arterial blood oxygen saturation of 90-97\% compared with $90-100 \%$ showed that in patients at risk of hypoxicischaemic brain injury 180 -day mortality was lower in the lower oxygen target group [74]; however, this difference was no longer statistically significant when adjusted for baseline differences [80]. A pilot RCT targeting a $\mathrm{PaO}_{2}$ of $10-15 \mathrm{kPa}$ compared with $20-25 \mathrm{kPa}$ showed no difference in the values of biomarkers of neurological injury [75]. Overall the evidence is mixed but suggests targeting normal oxygenation rather than hyperoxaemia.
Observational data suggest avoiding hypoxaemia but there are no RCTs on this topic.

In most post-cardiac arrest patients, controlled oxygenation will require tracheal intubation and mechanical ventilation for at least $24-72 \mathrm{~h}$, the exception being the completely conscious patient with a patent airway who should be treated with an oxygen mask or non-invasive ventilation targeting a peripheral oxygen saturation $\left(\mathrm{SpO}_{2}\right)$ of $94-98 \%$. During cardiac arrest, patients' lungs are ventilated with the maximum feasible inspired oxygen, which is usually $100 \%$ during advanced resuscitation [9]. After ROSC the goal should be to monitor oxygenation either with a pulse oximeter or preferably with an early arterial blood gas sample. Oxygenation measured early after ROSC is highly variable, varying from hypoxaemia to extreme hyperoxaemia [81]. Thus, it is appropriate to titrate the inspired oxygen by adjusting either the oxygen flow if using bag-mask ventilation or the fraction inspired oxygen $\left(\mathrm{FiO}_{2}\right)$ if using a mechanical ventilator [82]. Prolonged use of $100 \%$ inspired oxygen, for example during transport, will lead commonly to extreme hyperoxaemia [83]. Another method for monitoring is using cerebral oxygen monitoring with near infrared spectroscopy, but its role during post resuscitation care is uncertain $[84,85]$.

\section{Control of ventilation}

These guidelines are informed by the same ILCOR systematic review noted in the section on oxygenation [9, 66]. The ILCOR treatment recommendations in relation to ventilation are the following:

- There is insufficient evidence to suggest for or against targeting mild hypercapnia compared with normocapnia in adults with ROSC after cardiac arrest.

- We suggest against routinely targeting hypocapnia in adults with ROSC after cardiac arrest (weak recommendation, low-certainty evidence).

After ROSC, blood carbon dioxide values $\left(\mathrm{PaCO}_{2}\right)$ are commonly increased because of intra-arrest hypoventilation and poor tissue perfusion [86], causing a mixed respiratory acidosis and metabolic acidosis [87]. Carbon dioxide is a well-known regulator of blood vessel tone and cerebral blood flow [88]. Increased $\mathrm{PaCO}_{2}$ (hypercapnia) increases cerebral blood flow, cerebral blood volume and intracerebral pressure. Hypocapnia causes vasoconstriction that may decrease blood flow and cause cerebral ischaemia [89].

The main method for controlling $\mathrm{PaCO}_{2}$ in a mechanically ventilated patient is adjusting the minute volume by changing the ventilation frequency and or tidal volume. In general, limiting the tidal volume and using a lung 
protective ventilation strategy is the standard of care, especially in patients with acute respiratory distress syndrome (ARDS) [9, 90, 91]. Acute respiratory distress syndrome is not uncommon in cardiac arrest patients and is associated with worse outcomes $[9,92,93]$. Low lung compliance predicts poor functional outcome in OHCA patients [94]; however, ventilation with lower tidal volumes is not standard practice in neurointensive care [95].

Two pilot studies have compared different carbon dioxide targets during post resuscitation care $[75,96]$. One study found targeting mild hypercapnia $(50-55 \mathrm{mmHg}$ ) compared with normocapnia $(35-45 \mathrm{mmHg})$ resulted in lower neuron specific enolase (NSE) values, a marker of the magnitude of neurological injury [96]. Another pilot study compared the lower and higher end of the range for normocapnia (33-45 $\mathrm{mmHg}$ ) for the first $36 \mathrm{~h}$ of post resuscitation care and found no difference in markers of neurological injury [75]. Both of these studies showed that a higher $\mathrm{PaCO}_{2}$ was associated with higher cerebral oxygenation measured with near infrared spectroscopy (NIRS), but the clinical implications of this are uncertain [85]. Several large observational studies have aimed to define the optimal $\mathrm{CO}_{2}$ during post-cardiac arrest care [97-102]. The results are mixed, with some studies indicating harm from both hypo- and hypercapnia and some suggesting better outcome with mild hypercapnia. Recent UK observational data suggest a relationship between arterial oxygen and carbon dioxide. Data from the first $24 \mathrm{~h}$ of post resuscitation care observed a combination of hypoxia and hypocapnia was associated with a worse outcome and did not report harm from hyperoxia [103]. Previous observational data from Finnish ICUs reported similar findings [97].

Observational data suggest that patients undergoing TTM are prone to hypocapnia [104]. This may be avoided by frequent measurement of carbon dioxide with arterial blood gas analysis and use of end tidal $\mathrm{CO}_{2}$ monitoring. In patients undergoing TTM with lower temperature targets, $\mathrm{PaCO}_{2}$ management including measurement is particularly challenging [105]. There is limited evidence to support a particular method for measuring $\mathrm{PaCO}_{2}$ during hypothermia, therefore the guidance to use either a temperature or non-temperature corrected approach for measuring blood gases is based on expert opinion [106].

The recommendation for tidal volume is based on current guidance for lung protective ventilation in the ICU [107] and limited observational data from post cardiac arrest patients [108]. One observational study suggests that using a tidal volume of $6-8 \mathrm{~mL} \mathrm{~kg}^{-1}$ to ventilate the lungs of post-cardiac arrest patients may be associated with improved outcome [108]. This study also showed that by using higher ventilation frequency normocapnia may be achieved [108].

\section{Circulation}

\section{Coronary reperfusion}

Percutaneous coronary intervention following ROSC with ST-elevation

Arrhythmia caused by myocardial ischaemia is the commonest cause of sudden cardiac death (SCD) in adults $[109,110]$. Immediate reperfusion using percutaneous coronary intervention (PCI) of the culprit coronary lesion has been used for more than 20 years. This strategy is supported by many observational studies that reported a significant association between early PCI with survival and favourable neurological outcome after OHCA. Whilst the benefit of early PCI in OHCA caused by a recent coronary occlusion is universally acknowledged, the main challenge is to identify the best candidates for coronary angiography (CAG) among all resuscitated patients. In patients with ST segment elevation (STE) or left bundle branch block (LBBB) on the post-ROSC electrocardiogram (ECG) more than $80 \%$ will have an acute coronary lesion [111]. A systematic review completed for the 2015 ILCOR CoSTR identified 15 observational studies enrolling 3800 patients showing a mortality benefit for emergent versus delayed or no cardiac catheterisation among patients with ROSC after cardiac arrest with evidence of STE on their ECG [112]. The treatment recommendation from 2015 was to recommend emergency cardiac catheterisation laboratory evaluation in comparison with cardiac catheterisation later in the hospital stay or no catheterization in select adult patients with ROSC after OHCA of suspected cardiac origin with ST elevation on ECG (strong recommendation, low-quality evidence). The 2017 European Society of Cardiology Guidelines for the management of acute myocardial infarction with STsegment elevation state that 'a primary PCI strategy is recommended in patients with resuscitated cardiac arrest and an ECG consistent with STEMI' [113].

\section{Percutaneous coronary intervention following ROSC with-} out ST-elevation

In OHCA patients without ST segment elevation, several large observational series showed that absence of ST segment elevation does not completely exclude the presence of a recent coronary occlusion [114]. Therefore, the decision for early CAG should be based on meticulous patient assessment for the presence of haemodynamic or electrical instability and ongoing myocardial ischaemia taking into account multiple factors including previous medical history, warning symptoms before arrest, initial cardiac rhythm for CA [115], ECG pattern post ROSC, and echocardiography, as well as comorbidities. When an ischaemic cause is considered likely, a similar approach as for patients with STEMI should be followed. In patients with a low probability of an ischaemic cause of cardiac 
arrest, delaying CAG for few hours or days may buy time for initial management in ICU, enabling early initiation of post-resuscitation care (haemodynamic optimisation, protective ventilation, TTM) and prognostication. This 'wait and see' management may also avoid performing CAG in patients with the lowest probability of an acute coronary lesion. These two strategies (early versus delayed CAG) were evaluated in patients with VF arrest and without shock in an RCT that showed no difference in 90-day survival, the primary outcome [odds ratio 0.89 ; 95\% confidence interval (CI) $0.62-1.27 ; P=0.51$ ] [10], In this study, the median time to target temperature was $5.4 \mathrm{~h}$ in the immediate angiography group and $4.7 \mathrm{~h}$ in the delayed angiography group (ratio of geometric means 1.19; 95\% CI 1.04-1.36). Another recently published pilot RCT comparing early with delayed CAG also showed no difference in the primary outcome, which was a composite of efficacy and safety measures [116]. Further trials testing the same hypothesis are ongoing (DISCO NCT02309151, COUPe NCT02641626, TOMAHAWK NCT02750462, EMERGE NCT02876458). The 2020 European Society of Cardiology Guidelines for the management of acute coronary syndromes in patients without persistent ST-segment elevation state that 'delayed as opposed to immediate angiography should be considered in haemodynamically stable patients without ST-segment elevation successfully resuscitated after an out-of-hospital cardiac arrest' [11].

Ideally, coronary interventions would be undertaken only in those patients without permanent severe neurological injury. Patients with irreversible hypoxic-ischaemic brain injury are unlikely to benefit from PCI, even if a culprit coronary lesion is successfully treated [117]. However, the absence of a universally acceptable prognostic tool in the first hours after ROSC makes it impossible to identify such patients with high sensitivity and specificity at the time of hospital admission.

\section{Haemodynamic monitoring and management Haemodynamic monitoring}

Post-resuscitation myocardial dysfunction and low cardiac index may occur in up to $60 \%$ of post-cardiac arrest patients $[30,118]$ and may be even more common in patients with an acute myocardial infarction (AMI) as the cause of the arrest [119]. Early echocardiography can identify underlying cardiac pathology, quantify the degree of myocardial dysfunction and help guide haemodynamic management. Serial echocardiography or invasive monitoring with a pulmonary artery catheter quantifies myocardial dysfunction and indicates trends [28, 29, 120]. Impaired cardiac function is most common during the first 24-48 h after which it gradually resolves [30, 118]. Whether low cardiac output (or index) is associated with poor outcome is currently unclear. A sub-study of the TTM trial showed that low cardiac index may not be associated with outcome if lactate clearance is maintained [121]. These findings were independent of target temperature. Both non-invasive and invasive monitoring with echocardiography, arterial lines and measurement of cardiac output are commonly used in intensive care and it is reasonable to use these to guide treatment in cardiac arrest patients (best practice statement).

\section{Haemodynamic management: Mean arterial pressure and cerebral perfusion}

A systematic review completed for the 2015 ILCOR CoSTR searched for studies that compared titration of therapy to achieve a specific haemodynamic goal with no haemodynamic goal [122]. At that time, only observational studies were identified [123-127]. That systematic review also identified observational studies that compared a bundle of therapies with a specific blood pressure target with no bundle [128-130]. The 2015 CoSTR treatment recommendations were:

- We suggest haemodynamic goals (e.g. MAP, systolic blood pressure) be considered during post-resuscitation care and as part of any bundle of post-resuscitation interventions (weak recommendation, lowquality evidence).

- There is insufficient evidence to recommend specific haemodynamic goals; such goals should be considered on an individual patient basis and are likely to be influenced by post-cardiac arrest status and preexisting comorbidities (weak recommendation, lowquality evidence).

An evidence update for this topic was included in the 2020 ILCOR CoSTR and included two RCTs [9, 131, 132] and 11 observational studies [121, 133-142] published since the 2015 systematic review [122]. Two RCTs (including 232 patients) compared a blood pressure target of $65-75 \mathrm{mmHg}$ to $80-100 \mathrm{mmHg}$ with [131] and without [132] goal-directed optimisation of cardiac function. These studies were not powered for clinical outcomes but used surrogate markers of neurological injury such as MRI [131] and NSE [132]. Whilst these studies showed that higher MAP targets with vasopressors are safe, and do not, for example, lead to cardiac arrhythmias, they failed to show any clear improvement in surrogate markers of brain injury with a higher MAP target.

Nine observational studies found hypotension was associated with poor outcome [134-139, 141, 142]. One study found time spent below optimal MAP (assessed by correlation between near-infrared spectroscopy and blood pressure) was associated with poor outcome [133]; 
one study did not find low cardiac output to be associated with poor outcome [121] while the last study documented better outcomes among patients given fluids compared with vasopressors to increase MAP [140]. These observations are similar to the five observational studies included in the 2015 ILCOR Guidelines [122]. While hypotension $(<65 \mathrm{mmHg})$ is consistently associated with poor outcome, we do not have high certainty evidence to guide an optimal MAP target.

Mean arterial pressure (MAP) is one of the main determinants of cerebral blood flow (CBF) [143]. Although a high MAP is generally required in non-anoxic brain injured patients because of cerebral swelling and increased intracranial pressure (ICP) [144], few data on ICP values are available in cardiac arrest survivors. In many post-cardiac arrest patients, CBF autoregulation is impaired or the lower limit is right-shifted [133, 145]. This means that at lower MAP values, in some patients CBF may be MAP-dependent with an increased risk of cerebral hypoperfusion (i.e. hypotension) or hyperaemia and intracranial hypertension (i.e. hypertension).

The use of cerebral oxygen saturation or ICP monitoring to determine the presence of autoregulation and to determine an optimal MAP may enable a more individualised approach [146]. In a retrospective study, the estimated optimal MAP (i.e. MAP target at which the autoregulation is more effective) was $85 \mathrm{mmHg}$ in postcardiac arrest patients with preserved autoregulation and $100 \mathrm{mmHg}$ when the autoregulation was impaired [133]. Another small observational study calculated a median optimal MAP of $89 \mathrm{mmHg}$ in the same setting [147]. However, there are no prospective studies evaluating whether an autoregulation-driven MAP target may influence neurological injury and/or outcome. A more recent study has shown that after cardiac arrest, in particular in cases of non-cardiac origin, episodes of elevated ICP and/or brain hypoxia are frequent and a higher MAP is necessary to improve brain oxygenation [147]. Preliminary evidence based on measurement of brain tissue oxygenation $\left(\mathrm{PbtO}_{2}\right)$ has shown that in resuscitated comatose patients impairment of oxygen diffusion to the brain may cause persisting brain hypoxia despite optimisation of oxygen delivery to the brain [148]. The implementation and the safety of these invasive monitoring tools in cardiac arrest patients need to be further evaluated. While these are all observational findings, they indicate optimal MAP targets may need to be individualised and support further research into identification of optimal MAP targets for individual cardiac arrest survivors receiving intensive care. In the post cardiac arrest patient, transcranial Doppler (TCD) can give information about cerebral haemodynamics and, in the future, may have a role in optimising haemodynamics in these patients
[149]. Changes in cerebral blood flow can be seen using TCD and this may be a target to for treatment [150-152]. However, the technique and interpretations of the images is operator dependent and requires an acoustic window in the patient. Moreover, cerebral haemodynamics are continuously changing and serial measurements are possible only intermittently and the monitoring is labourintensive. Based on the evidence summarised by ILCOR [9] we suggest avoiding hypotension ( $\mathrm{MAP}<65 \mathrm{mmHg}$ ) and targeting MAP to achieve adequate urine output $\left(>0.5 \mathrm{~mL}^{-1} \mathrm{~kg} \mathrm{~h}^{-1}\right)$ and normal or decreasing lactate values (best practice statement).

\section{Haemodynamic management: Heart rate}

Tachycardia was associated with poor outcome in one retrospective study [153]. During mild induced hypothermia the normal physiological response is bradycardia. In animal models this has been shown to reduce the diastolic dysfunction that is usually present early after cardiac arrest [154]. Bradycardia was previously considered to be a side effect, especially below a rate of $40 \mathrm{~min}^{-1}$; however, bradycardia has been shown to be associated with a good outcome $[155,156]$. Similar association between bradycardia and improved longterm outcome has been shown in patients not treated with TTM [157].

Sedation, controlled ventilation and a temperature between 32 and $36{ }^{\circ} \mathrm{C}$ lower oxygen consumption in cardiac arrest patients. Although bradycardia generally reduces cardiac output, this is well tolerated in this post-arrest setting. We suggest bradycardia (heart rate $<30-40 \mathrm{~min}^{-1}$ ) be left untreated as long as there are no signs of hypoperfusion (i.e. increasing lactate, reduced urinary output etc.) (best practice statement).

Haemodynamic management: Fluid resuscitation, vasoactive and inotropic drugs

There is limited evidence to guide optimal fluid therapy for post-cardiac arrest patients. One study during which invasive monitoring and filling pressures were used observed that up to 5-7 L of fluid were given during the first $24 \mathrm{~h}$ [30]. One retrospective study indicated that with a treatment algorithm involving the pulse contour continuous cardiac output (PiCCO) system larger fluid volumes (range $4-5 \mathrm{~L}$ during the first $24 \mathrm{~h}$ ) were associated with a lower incidence of acute kidney injury [158].

There is little direct evidence comparing various vasoactive drugs for post-cardiac arrest patients; therefore, this recommendation is based on indirect evidence from critically ill patients in general. The most recent Cochrane review on vasopressors for hypotensive shock included 28 RCTs ( $n=3497$ patients) and did not find any mortality benefit from any of the six vasopressors 
assessed. Acknowledging noradrenaline as the most commonly used vasopressor, their suggestion was that major changes in clinical practice were not needed [159]. As noradrenaline is the most widely used vasoactive agent for post-cardiac arrest patients, we suggest using noradrenaline as the first-line vasoactive agent in hypotensive post-cardiac arrest patients. A recent RCT comparing noradrenaline with adrenaline in 57 patients with acute myocardial infarction and cardiogenic shock was terminated early because of significantly more refractory shock in patients treated with adrenaline [160]. The COMACARE and NEUROPROTECT pilot trials also used noradrenaline as the drug of choice to achieve higher MAP targets [131, 132]. None of the studies showed any evidence of relevant tachycardia, arrhythmias or recurrent shock in the higher MAP group, despite the use of significantly higher doses of noradrenaline compared with the lower MAP group. This suggests that noradrenaline is well tolerated in post-cardiac arrest patients [131].

Post-resuscitation myocardial dysfunction often requires inotropic support. Based on experimental data, dobutamine is the most established treatment in this setting $[161,162]$, but the systemic inflammatory response that occurs frequently in post-cardiac arrest patients also causes vasoplegia and severe vasodilation [30], The NEUROPROTECT trial used dobutamine to increase cardiac index in the higher MAP group. Although this did not decrease neurological injury it also did not increase myocardial injury [131].

\section{Steroids}

ILCOR performed an evidence update on use of steroids for post-cardiac arrest patients for the 2020 guidelines [9]. Three small RCTs and a large observational study have addressed the use of steroids in post-cardiac arrest patients [163-166]. Two of the RCTs used steroids both during CPR for IHCA and after ROSC [163, 164]. The first of these RCTs showed improved survival to discharge with a combination of methylprednisolone, vasopressin and adrenaline during cardiac arrest and hydrocortisone after ROSC for those with shock, compared with the use of only adrenaline and placebo [9/48 (19\%) versus 2/52 (4\%); RR 4.87; 95\% CI 1.17-13.79] [164]. The second RCT showed improved survival to discharge with favourable neurological outcome with methylprednisolone, vasopressin and adrenaline during cardiac arrest, and hydrocortisone in those with postROSC shock compared with only adrenaline and placebo [18/130 (13.9\%) versus 7/138 (5.1\%); RR 2.94; 95\% CI 1.16-6.50] [163]. Only the third RCT confined the use of steroids to the post-resuscitation phase; it did not show any benefit for steroid post-ROSC but included only 50 patients [166].

One trial has recently been completed but is not yet published (NCT02790788). ILCOR recommended a systematic review be undertaken once the recently completed trial is published and, therefore, left the treatment recommendation unchanged from 2010 [167]:

- There is insufficient evidence to support or refute the use of corticosteroids for patients with ROSC following cardiac arrest.

Until there is higher-certainty evidence supportive of their use, we suggest that steroids are not given routinely to post-cardiac arrest patients (weak recommendation, low-certainty evidence).

\section{Potassium}

Hyperkalaemia is common immediately after cardiac arrest. Subsequent endogenous catecholamine release and correction of metabolic and respiratory acidosis promotes intracellular transportation of potassium, causing hypokalaemia. Hyperkalaemia in the post-cardiac arrest period is associated with worse outcome [168]: hypokalaemia, on the other hand may predispose to ventricular arrhythmias. Based on these observational studies we suggest that potassium be given to maintain the serum potassium concentration between 4.0 and $4.5 \mathrm{mmol} \mathrm{L}^{-1}$ (best practice statement).

\section{Mechanical circulatory support}

If treatment with fluid resuscitation, inotropes and vasoactive drugs is insufficient to support the circulation, consider insertion of a mechanical circulatory assistance device (e.g. IMPELLA, Abiomed, USA) [126, 169, 170]. One study indicated that $10-15 \%$ of patients with $\mathrm{OHCA}$ and ongoing cardiogenic shock eventually require mechanical circulatory support [171]. In patients with cardiogenic shock without cardiac arrest some centres still advocate use of an intra-aortic balloon pump (IABP), although the IABP-SHOCK II Trial failed to show that use of the IABP improved 30-day mortality in patients with myocardial infarction and cardiogenic shock [172, 173]. One recent small RCT found no difference in outcome in patients with acute myocardial infarction and cardiogenic shock treated with an IMPELLA device compared with an IABP [174]. Another retrospective study including only post-cardiac arrest patients found no difference in clinical outcome but higher incidence of bleeding with the use of IMPELLA compared with IABP [169]. Thus far, the evidence about which type of mechanical device is superior appears inconclusive and thus their use should be decided on a case-by-case basis. 
The 2015 ESC Guidelines for the management of patients with ventricular arrhythmias and the prevention of sudden cardiac death include the following recommendation for the use of mechanical circulatory support: left-ventricular assist devices or arterio-venous extra corporal membrane oxygenation should also be considered in haemodynamically unstable patients with acute coronary syndromes (ACS) and recurrent ventricular tachycardia (VT) or ventricular fibrillation (VF) despite optimal therapy [175].

\section{Implantable cardioverter defibrillators}

An implantable cardioverter defibrillator (ICD) is a device used for the treatment of certain life-threatening arrhythmias. The European Society of Cardiology has published guidelines on the indications for ICD therapy [175]. An ICD may be implanted for primary or secondary prevention. The former applies to those who have not experienced a dangerous arrhythmia but who are considered at high risk of one. This group includes patients with cardiomyopathies, inherited primary arrhythmic syndromes, congenital heart disease but also individuals with primary arrhythmias in structurally normal hearts $[176,177]$. Secondary prevention refers to patients who have already survived a dangerous arrhythmic event and are still considered at risk of further events. Careful selection of patients is needed to identify those who may benefit from ICD implantation and whose lives can be prolonged by preventing arrhythmic SCD.

\section{Disability (optimising neurological recovery) Control of seizures}

Seizures are reported in $20-30 \%$ of cardiac arrest patients in the ICU and are usually a sign of a severe hypoxicischaemic brain injury. Seizures may be observed as clinical convulsions (clinical seizure) and/or as typical activity in the EEG (electrographic seizure).

Myoclonus are sudden, brief, shock-like involuntary muscle contractions and by far the most common type of clinical seizure in post-arrest patients $[178,179]$. It is often generalised but may be focal (periodic eye-opening, swallowing, diaphragmic contractions etc.) or multi-focal [180]. It typically develops during the first $1-2$ days after the arrest and is often transient during the first daysweek. It is associated with a poor prognosis but some patients survive with a good outcome [181, 182]. Most post-hypoxic myoclonus has a cortical origin [183] and the EEG shows synchronous time-locked discharges or burst-suppression in a substantial proportion of patients [181].
Focal and generalised tonic-clonic seizures also occur after cardiac arrest, and it is not uncommon that an individual patient has several seizure sub-types [178].

Lance-Adams syndrome is a less frequent form of myoclonus usually developing in a patient who has regained consciousness [184, 185]. It is more common after hypoxic cardiac arrest and mainly affects the limbs where it is induced by purposeful actions or sensory stimulation. It may be disabling and often becomes chronic [182].

Some of the evidence informing this guideline is set out in a systematic review that informed the ILCOR 2015 CoSTR [122] and updated in 2020 [9]. The 2020 updated treatment recommendations are as follows:

- We suggest against seizure prophylaxis in adult postcardiac arrest survivors (weak recommendation, very-low certainty evidence).

- We suggest treatment of seizures in adult post-cardiac arrest survivors (weak recommendation, verylow certainty evidence).

Studies using continuous EEG-monitoring reveal that electrographic epileptiform activity and clinical convulsions are equally common and that there is a substantial overlap [186]. The evaluation of electrographic seizures is often confounded by the concomitant effects of brain injury, metabolic factors and sedation, making possible clinical correlates and effects of treatment harder to evaluate. New definitions of electrographic status epilepticus have been published recently by the American Clinical Neurophysiology Society (ACNS) [187]. The ACNS uses strict and conservative criteria which are typically not fulfilled by post-arrest patients [186]. Instead, most of these patients have EEG-patterns that may or may not be defined as electrographic 'seizures' or, if prolonged as 'status epilepticus', and depend on the local EEG-interpreter.

Sedative drugs have potent seizure-suppressing effects and are recommended as third-line treatment of status epilepticus. Propofol and benzodiazepines are used routinely during the first days after cardiac arrest while the patient is mechanically ventilated and treated with TTM. Depending on the dosing, these drugs will suppress clinical myoclonus and epileptiform activity in the EEG [188, 189]. The seizures may be unmasked during sedation holds. There is limited evidence that conventional antiepileptic drugs (mainly valproate and levetiracetam) suppress epileptic activity on the EEG of post cardiac arrest patients [190]. These drugs are known to supress myoclonus of other origins [191]. Phenytoin and the pro-drug fosphenytoin are still used widely for the treatment of status epilepticus. In post-cardiac arrest patients, however, their negative inotropic and vasodilating effects makes 
them less suitable [192]. In a recently reported trial, valproate, levetiracetam and fosphenytoin were equally effective in terminating convulsive status epilepticus but fosphenytoin caused more episodes of hypotension [12].

There is currently no evidence supporting prophylactic treatment with antiepileptic drugs in the post-arrest setting. Previous studies on the effects of bolus-doses of thiopental [193] and diazepam/magnesium [194] after resuscitation showed no benefit in terms of survival or neurologic function but these studies were designed to investigate neuroprotection, not seizure suppression. Whether treatment of detected clinical and electrographic seizures alters patient outcome has not previously been studied in a randomised fashion but a multicentre trial of aggressive treatment of post-anoxic status epilepticus is currently ongoing [195]. In case series, $4-44 \%$ of patients with post-anoxic status epilepticus had a good outcome [196-199]. These patients were usually treated with multiple anti-epileptic drugs and had a delayed awakening, often beyond 2 weeks.

The EEG is an important tool to detect corresponding electrographic seizure activity in a patient with observed clinical convulsions and to monitor treatment effects. Shivering is a common seizure mimic during TTM. Active treatment of status epilepticus usually necessitates repeated routine EEGs or continuous EEG-monitoring. The relative benefit of continuous EEG compared with routine EEG has not been shown. Continuous EEG monitoring is labour intensive and likely to add significant cost to patient care. The net cost-effectiveness of this approach is controversial and may depend substantially on the setting [200, 201].

Since post-anoxic seizures and status epilepticus are manifestations of hypoxic-ischaemic brain injury, an assessment of the prognosis and potential for an eventual good outcome are central components of a treatment strategy. The EEG-background pattern is important but may sometimes be difficult to assess if there are concomitant abundant discharges. A continuous, normal voltage and reactive EEG background are benign features whereas a burst-suppression pattern or a suppressed background without reactivity are features related to worse prognosis $[181,199]$. Early onset $(<24$ h) of electrographic seizures, before the recovery of a continuous background is associated with worse prognosis [197, 202, 203]. In these patients, the EEG is often affected by the ongoing treatment. It is therefore suggested that additional information is obtained on the severity of brain injury from methods not significantly affected by sedative and anti-epileptic drugs such as somatosensory evoked potentials, serum NSE and neuroradiological investigations (preferably MRI).
Seizures may increase the cerebral metabolic rate and have the potential to exacerbate brain injury caused by cardiac arrest: treat seizures with levetiracetam and/or sodium valproate. Consider possible drug interactions. After the first event, start maintenance therapy. Additional treatment options include perampanel, zonisamide or topiramate. Consider increased dose of propofol or benzodiazepines to suppress myoclonus and electrographic seizures. Thiopental or phenobarbital may be considered in selected patients.

Treatment with sedatives and conventional antiepileptic drugs in high doses may delay awakening, prolong the need for mechanical ventilation, and increase critical care length of stay [204]. Consider that generalised myoclonus in combination with epileptiform discharges may be early signs of Lance-Adams syndrome which is compatible with awakening and a good outcome [181, 184]. In such cases, aggressive treatment may confound the clinical examination and lead to overly pessimistic prognostication.

\section{Temperature control}

A comprehensive systematic review of TTM was conducted for the 2015 COSTR [122, 205-207]. Following an evidence review for the 2020 CoSTR, these ILCOR treatment recommendations remained unchanged from 2015 [9].

- We recommend selecting and maintaining a constant target temperature between 32 and $36{ }^{\circ} \mathrm{C}$ for those patients in whom temperature control is used (strong recommendation, moderate-quality evidence). Whether certain subpopulations of cardiac arrest patients may benefit from lower $\left(32-34{ }^{\circ} \mathrm{C}\right)$ or higher $\left(36{ }^{\circ} \mathrm{C}\right)$ temperatures remains unknown, and further research may help elucidate this.

- We recommend targeted temperature management as opposed to no targeted temperature management for adults with OHCA with an initial shockable rhythm who remain unresponsive after ROSC (strong recommendation, low-quality evidence).

- We suggest targeted temperature management as opposed to no targeted temperature management for adults with OHCA with an initial non-shockable rhythm who remain unresponsive after ROSC (weak recommendation, very-low-quality evidence).

- We suggest targeted temperature management as opposed to no targeted temperature management for adults with IHCA with any initial rhythm who remain unresponsive after ROSC (weak recommendation, very-low-quality evidence). 
- We suggest that if TTM is used, duration should be at least $24 \mathrm{~h}$ (weak recommendation, very-low-quality evidence).

- We recommend against routine use of prehospital cooling with rapid infusion of large volumes of cold IV fluid immediately after ROSC (strong recommendation, moderate-quality evidence).

- We suggest prevention and treatment of fever in persistently comatose adults after completion of TTM between 32 and $36^{\circ} \mathrm{C}$ (weak recommendation, verylow-quality evidence).

\section{Treatment of fever}

The definition of fever varies in different studies and no specific evaluation of the cause (i.e. ischaemia-reperfusion, neurogenic fever, infection) is generally reported. A large observational study investigating serial measurements in more than 35,000 individuals concluded that mean body temperature measured in the oral cavity was $36.6{ }^{\circ} \mathrm{C}\left(99 \%\right.$ ranges $\left.35.3-37.7^{\circ} \mathrm{C}\right)$ in healthy adults [208]. A reasonable definition of fever is, therefore, body temperature above $37.7^{\circ} \mathrm{C}$, as recently used in a large randomised cardiac arrest trial [14]. However, this definition in critically ill patients usually relies on measurement of 'core' temperature (i.e. blood, bladder, oesophagus) and is only an estimation of brain temperature, which could exceed it by $0.4-2.0^{\circ} \mathrm{C}$ [209].

Fever is common during the first 2-3 days after cardiac arrest and is associated with worse outcomes in observational studies [210]. Fever following TTM (i.e. induction of hypothermia at $32-36{ }^{\circ} \mathrm{C}$ ) is also called rebound hyperthermia and is associated with worse outcomes, in particular with high temperatures [211, 212]. Whether fever contributes to poor neurological outcome or is just a marker of severe brain injury remains unknown. To date, no randomised trial has compared controlled normothermia (i.e. keeping target temperature below $37.8^{\circ} \mathrm{C}$ ) with no fever control.

\section{Targeted Temperature Management: Cooling versus nor- mothermia}

A meta-analysis shows that mild induced hypothermia is neuroprotective and improves outcomes in animal models of cardiac arrest [213]. The authors conclude that there may be translational gaps because research on large (gyrencephalic) and comorbid animals is uncommon. The theoretical background that lowering core temperature suppresses several detrimental pathways leading to neuronal death is well established, but the specific mechanisms of hypothermic neuroprotection remain unclear [214]. Hypothermia decreases the cerebral metabolic rate for oxygen $\left(\mathrm{CMRO}_{2}\right)$ by about $6 \%$ for each $1{ }^{\circ} \mathrm{C}$ reduction in core temperature and this reduces the release of excitatory amino acids and the production of free radicals $[215,216]$. In the temperature range of $33-36{ }^{\circ} \mathrm{C}$, however, there is no difference in the inflammatory cytokine response in adult patients [217].

All studies evaluating post-cardiac arrest patients and mild induced hypothermia included only patients with altered consciousness (i.e. Glasgow Coma Scale $<9$ ). One randomised trial and a quasi-randomised trial demonstrated improved neurological outcome at hospital discharge or at 6 months in comatose patients after out-ofhospital witnessed cardiac arrest with an initial shockable rhythm [218, 219]. Cooling was initiated within minutes to hours after ROSC and a target temperature of $32-34{ }^{\circ} \mathrm{C}$ was maintained for $12-24 \mathrm{~h}$. These two trials represented the beginning of modern post-cardiac arrest care. More recently, a French multicentre trial randomised 581 adult patients who were comatose after resuscitation of either an IHCA or OHCA with an initial non-shockable rhythm (i.e. asystole or pulseless electrical activity) to either TTM with a target temperature of $33^{\circ} \mathrm{C}$ or a target temperature of $37^{\circ} \mathrm{C}$, for $24 \mathrm{~h}$ [13]. The use of TTM at $33^{\circ} \mathrm{C}$ led to a higher percentage of patients who survived with a favourable neurological outcome at day 90 , assessed as a cerebral performance category score (CPC) of $1-2,(10.2 \%$ versus $5.7 \%$, difference $4.5 \%$; $95 \%$ CI $0.1-8.9 ; P=0.04)$, while mortality did not differ $(81.3 \%$ versus $83.2 \%$, difference $1.9 ; 95 \% \mathrm{CI}-8.0$ to 4.3$)$. The benefit of a lower target temperature was more evident in patients with shorter time to ROSC ( $<15 \mathrm{~min})$ and among in-hospital cardiac arrest patients. These results differ from a previous retrospective registry study of 1830 patients with non-shockable OHCA where poor neurological outcome was more common among those receiving mild induced hypothermia [adjusted OR 1.44 (95\% CI 1.04-2.01)] [220]. The ongoing targeted hypothermia versus targeted normothermia after OHCA (TTM2) trial will compare a target temperature of $33^{\circ} \mathrm{C}$ with strict normothermia $\left(<37.8^{\circ} \mathrm{C}\right)$ during a 40-h intervention period in 1900 patients and will address the effectiveness of cooling procedures in cardiac arrest patients in comparison with fever management [14].

\section{Targeted Temperature Management: Timing to initiate hypothermia}

Animal data suggest that TTM should be initiated as soon as possible [221], although delays of several hours seem to be neuroprotective in several species [213]. Early cooling, i.e. initiated in the pre-hospital field after ROSC, has been tested in some RCTs [222, 223]; although target temperature could be achieved faster than with standard in-hospital cooling, no significant effect on patient outcomes was reported. Moreover, in one study pre-hospital use of cold fluids to induce early hypothermia was associated with more re-arrests in the field and more frequent 
pulmonary oedema on admission than the control group [224].

Intra-arrest hypothermia (i.e. initiated during CPR) has been proposed as an effective method to provide TTM; however, use of cold fluids during CPR in a large RCT including OHCA patients showed no improvement in outcome from this strategy and even a decreased ROSC rate in patients with an initial shockable rhythm [224]. One small feasibility trial [225] and one RCT [226] have tested the use of trans-nasal evaporative cooling, which could induce rapid cooling in OHCA patients. Both trials reported no significant benefits on patient outcomes, although in the latter trial a post-hoc analysis of the subgroup of patients with an initial shockable rhythm and in whom cooling was initiated $<20 \mathrm{~min}$ from collapse showed improved neurological outcome at 90 days [226, 227].

Targeted Temperature Management: Optimal target temperature during hypothermia

The Targeted Temperature Management after Cardiac Arrest trial (TTM-trial) randomised 950 OHCA patients with both initial shockable and non-shockable rhythms to a strategy including $36 \mathrm{~h}$ of temperature control (i.e. $28 \mathrm{~h}$ at target temperature followed by slow rewarming) and fever control up to $72 \mathrm{~h}$ after randomization; the two target temperatures during the intervention phase were $33{ }^{\circ} \mathrm{C}$ or $36{ }^{\circ} \mathrm{C}$ [27]. Strict protocols were followed for assessing prognosis and for withdrawal of life-sustaining treatment (WLST). There was no difference in the primary outcome [i.e. all-cause mortality; hazard ratio 1.06 (95\% CI $0.89-1.28)]$ or in neurological outcome at 6 months [relative risk $1.02(0.88-1.16)]$. Neurological outcome and cognitive function were also similar [228, 229], as were brain injury biomarker values $[230,231]$. TTM at $33{ }^{\circ} \mathrm{C}$ was associated with decreased heart rate, elevated lactate, the need for increased vasopressor support, and a higher extended cardiovascular SOFA score compared with TTM at $36^{\circ} \mathrm{C}[136,232]$. A small three-armed randomised trial compared $32{ }^{\circ} \mathrm{C}$ with $33^{\circ} \mathrm{C}$ and $34^{\circ} \mathrm{C}$ and found no difference in good neurological outcome, assessed as a modified Rankin Score (mRS) of 0-3 at day 90 [62.3\% (95\% CI $48.3-76.6)$ vs $68.2 \% 95 \%$ CI $52.4-81.4)$ vs $65.1 \%$ (95\% CI 49.0-79.0)] [233].

Since the publication of previous guidelines, many sites have changed to a target temperature of $36{ }^{\circ} \mathrm{C}$ in routine practice $[234,235]$. There have been reports that a change to $36^{\circ} \mathrm{C}$ has led to worse temperature control and more early fever [236], but there are other reports showing good compliance with a $36{ }^{\circ} \mathrm{C}$-protocol and a possible clinical advantage, such as earlier awakening and less sedative use [237]. Results from two large registry analyses, one from the cardiac arrest registry to enhance survival (CARES) surveillance group in the US [238] and one from the Australian and New Zealand Intensive Care Society Centre for Outcome and Resource Evaluation (ANZICS-CORE) [239] indicate that a widespread change in TTM-use has occurred after publication of the TTM-trial, with a rising average lowest temperature in the ICU and reduced use of TTM. Furthermore, survival decreased, but was not statistically associated with a decreased use of TTM [238]. In this setting, the optimal temperature during mild induced hypothermia is therefore unknown and more high-quality large trials are needed [240].

Targeted Temperature Management: Duration of hypothermia

The optimal duration for mild induced hypothermia and TTM is unknown although the period of hypothermia is most commonly $24 \mathrm{~h}$. Previous trials treated patients with 12 to $28 \mathrm{~h}$ of TTM [27, 218, 219]. Two observational trials found no difference in outcomes with $24 \mathrm{~h}$ compared with $72 \mathrm{~h}$ of TTM [241, 242]. A recent randomised trial $(n=351)$ investigated TTM at $33^{\circ} \mathrm{C}$ during $48 \mathrm{~h}$ or $24 \mathrm{~h}$ in unconscious patients after OHCA [243]. There was no significant difference in poor neurological outcome between groups (absolute difference 4.9\%; relative risk (RR) for a cerebral performance category $1-2$ at 6 months 1.08, 95\% CI 0.93-1.25). Adverse events were more common in the prolonged cooling group (RR 1.06, 95\% CI 1.01-1.12).

Contraindications to targeted temperature management Within the recommended TTM range of $32-36{ }^{\circ} \mathrm{C}$, there are few, if any, recognised contraindications. Results from a post hoc analysis of the TTM-trial suggest that if there is severe cardiovascular impairment at $33{ }^{\circ} \mathrm{C}$ a higher temperature might be targeted [232].

\section{Other therapies to improve neurological outcome}

In contrast to a number of positive results from studies in experimental settings [18], several neuroprotective drugs failed to demonstrate a positive clinical effect [164, 193, 194, 244-247]. More recently, erythropoietin [248], cyclosporine [249] and exenatide [250], used alone, or as an adjunct to mild induced hypothermia, have also not been shown to increase neurologically intact survival when included in the post arrest treatment of cardiac arrest patients. The combination of xenon and mild induced hypothermia, which is beneficial and superior to mild induced hypothermia alone in experimental settings [18, 251], has been studied in several trials with no convincing effects [252-254] and is undergoing further clinical evaluation (XePOHCAS, EudraCT Number 2017-00251432). Moreover, volatile anaesthetic drugs 
have demonstrated positive effects on cardiac and cerebral recovery in experimental settings [255], and clinical feasibility studies [256-258], but outcome data are lacking. Most recently, it has been shown that pig brain cells can survive and show electrical activity for more than 4-6 h after decapitation, when reperfusion of the brain was performed in highly artificial experimental settings [259]. Very specific extracorporeal life support concepts (i.e. controlled reperfusion of the whole body) have also demonstrated good neurological survival following 15-20 min of experimental cardiac arrest and in humans $[260,261]$. These concepts are currently also undergoing further clinical evaluation [262].

\section{General intensive care management}

There has been a recent systematic review and an ILCOR CoSTR on the subject of prophylactic antibiotics $[9,263]$. The ILCOR recommendation states the following:

- We suggest against the use of prophylactic antibiotics in patients following ROSC (weak recommendation, low certainty of evidence).

The remaining guidelines for the general ICU management of post-cardiac arrest patients are based on expert opinion. Most aspects of post cardiac arrest care follow general ICU practices. Some differences and nuances are inherent. Few aspects of general intensive care have been studied separately in the cardiac arrest population, but cardiac arrest patients have been included in trials on general intensive care practices. Specific features of the post cardiac arrest patients include the risk of brain injury and need to apply neurointensive care principles, the high occurrence of myocardial dysfunction, the use of anticoagulants and anti-platelet drugs and the high risk of aspiration pneumonitis among others. The typical length of stay in cardiac arrest patients will vary from 3 days to several weeks because of differences in time to awakening. This will influence certain aspects of care such as the initiation of and management of nutrition.

Many post cardiac arrest patients will require appropriate sedation and pain management, particularly those who are treated with TTM. During TTM, shivering is common-this can be managed with opioids and sedation. TTM influences the metabolism of several drugs and effects are in general prolonged. One RCT has compared the use of propofol and fentanyl with midazolam and fentanyl [264]. In a trial of 59 patients, the use of propofol and remifentanil resulted in shorter time to awakening but was associated with more frequent need of noradrenaline [264]. Similar findings have been shown in observational studies [265]. Sedation breaks are best initiated after TTM and rewarming has been completed.
Routine use of neuromuscular blocking drugs has been shown to be beneficial in observational studies [266, 267], but one small randomised pilot trial failed to show any such benefit [268]. In patients with ARDS and critical hypoxaemia, a meta-analysis has shown beneficial effects on outcome with the use of neuromuscular blockers [269]. Thus, in patients with critical hypoxaemia and ARDS following cardiac arrest, the use of a neuromuscular blocker may be considered, given the evidence for their use in ARDS. Patients should be nursed $30^{\circ}$ headup. This may decrease intracranial pressure (ICP) and decrease the risk of aspiration pneumonia. Many patients are at high risk of developing aspiration and ventilatorassociated pneumonia [270]. A recent RCT examined the prophylactic use of antibiotics in OHCA patients [271]. Whilst the study showed a decrease in ventilator associated pneumonia it did not find any other differences in other clinical outcomes; therefore, prophylactic antibiotics are not recommended. However, antibiotics can be considered in cases with clear suspicious infiltrates on the chest X-rays.

Patients require a nasogastric tube to decompress any abdominal distension. One small observational study has indicated that low-dose enteral feeding is tolerated during TTM after OHCA [272]. Gastric feeding may be initiated at low rates (trophic feeding) during TTM and increased after rewarming if indicated. If TTM of $36^{\circ} \mathrm{C}$ is used as the target temperature, gastric feeding rates may be increased early during TTM.

Routine use of ulcer prophylaxis in intensive care patients does not decrease mortality [273, 274]. However, a recent meta-analysis showed that in high-risk patients, the use of ulcer prophylaxis decreased gastrointestinal bleeding [275]. Post-cardiac arrest patients are likely to be at higher risk than general ICU patients given the use of anticoagulant and antiplatelet agents both pre and post arrest [276]. Therefore, it appears reasonable to administer stress ulcer prophylaxis in post-cardiac arrest patients, especially in those with coagulopathy [35].

Unless patients receive anticoagulation because of a myocardial infarction or ischaemia, deep venous thrombosis prophylaxis is recommended in critically ill patients $[277,278]$. The use of antiplatelet drugs do not prevent DVTs [279]. Out-of-hospital cardiac arrest patients are at risk for developing DVTs, especially if treated with TTM [280]. These appear more common in those treated with an invasive TTM device, likely related to catheter placement in the femoral vein [281]. No specific evidence exists on DVT prophylaxis in cardiac arrest patients. Thus, treatment should be individualised and be based on general ICU recommendations [277].

Hyperglycaemia is common after OHCA [168]. Hyperglycaemia is best managed with continuous infusion 
of insulin. The 2019 Guidelines of the American Diabetes Association recommend a target glucose range of 7.8-10.0 $\mathrm{mmol} \mathrm{L}^{-1}\left(140-180 \mathrm{mg} \mathrm{dL}^{-1}\right)$ for the majority of critically ill patients [282]. Tight glucose control does not appear to convey benefit and may be associated with hypoglycaemia $\left(<4.0 \mathrm{mmol} \mathrm{L}^{-1}\left(<70 \mathrm{mg} \mathrm{dL}^{-1}\right)\right.$ [283], which is harmful in critically ill patients [284]. In general, glucose-containing solutions are not recommended in patients with brain injury [285], but they may be needed to treat hypoglycaemia [284].

\section{Prognostication}

About two-thirds of in-hospital deaths in patients who are admitted to an intensive care unit in a coma following resuscitation from OHCA are caused by hypoxic-ischaemic brain injury [23, 24]. In a minority of cases these deaths occur as a direct consequence of hypoxic-ischaemic brain injury which results in an irreversible loss of all brain function, i.e. brain death [286]. However, most of these neurological deaths result from active withdrawal of life-sustaining treatment (WLST) in patients where the severity of hypoxic-ischaemic brain injury indicates that survival with a poor neurological outcome is very likely $[26,287]$. Accurate prognostication is, therefore, essential in order to avoid an inappropriate WLST in patients who still have a chance of a neurologically meaningful recovery and to avoid futile treatment in patients with a severe and irreversible neurological injury.

\section{Outcomes measures in neuroprognostication studies}

Neurological outcome after cardiac arrest is most commonly reported using Cerebral Performance Categories (CPC) [288]. The CPC is expressed as a five-point scale: CPC 1 (no or minimal neurological disability); CPC 2 (minor neurological disability); CPC 3 (severe neurological disability); CPC 4 (persistent vegetative state); and CPC 5 (death). Another commonly used outcome measure is the modified Rankin Score (mRS) [289], which includes 7 scores, from 0 (no symptoms) to 6 (dead). In 2018, a statement from ILCOR [290] suggested using $\mathrm{mRS}$ rather than $\mathrm{CPC}$ for measuring functional recovery after cardiac arrest, because mRS is more suitable than CPC for discriminating between mild and moderate disability [291,292] and has a substantial interrater reliability [293]. However, most studies on neurological prognosis after cardiac arrest still use CPC.

For both clarity and statistical purposes in studies on neuroprognostication after cardiac arrest the outcome is dichotomised as 'good' or 'poor'. However, there is no universal consensus on what represents a poor neurological outcome. Up to 2006, most neuroprognostication studies reported CPC 4 or 5 (vegetative state or death) as a poor outcome, and CPC from 1 to 3 (from absent to severe neurological disability) as a good outcome, while after that date an increasing number of studies included CPC 3 (severe neurological disability) among poor neurological outcomes [294]. In a recent systematic review [15], among 94 total studies on neurological prognostication after cardiac arrest, 90 (96\%) defined poor neurological outcome as CPC 3-5 and only four defined poor outcome as CPC $4-5$.

In prognostic accuracy studies, a predictor (index test) is assessed for its ability to predict an outcome. This design is like that of diagnostic accuracy studies. However, while in diagnostic accuracy studies the index test is evaluated against another test which represents the reference standard, or gold standard, prognostic accuracy studies evaluate the index test against the occurrence of the predicted event (target condition) after test recording [295]. When test results are expressed in binary format (i.e. positive vs. negative) the accuracy is expressed using sensitivity and specificity, which measure the ability of the test to identify those who will develop or not develop the target condition, respectively. Since most neuroprognostic tests predict poor neurological outcome, having a high specificity (i.e. a very-low rate of falsely pessimistic predictions potentially leading to an inappropriate WLST) is desirable. Ideally, an index test should be $100 \%$ specific, i.e. its false positive rate (FPR) should be zero, but this is difficult to achieve in practice. There is no universal consensus on how specific an index test should be for neuroprognostication after cardiac arrest. In a recent survey of 640 healthcare providers, the majority (56\%) felt an acceptable FPR for WLST from patients who might otherwise have recovered was $\leq 0.1 \%$ [296]. Along with the absolute value of specificity, precision of its estimate is important. A very specific test predicting poor outcome is of little clinical use when its precision is low, [i.e., when the confidence intervals (CIs) around the point estimate of its specificity are wide], because this indicates a high degree of uncertainty around the estimated specificity. In the 2014 ERC-ESICM Advisory Statement on neuroprognostication after cardiac arrest [297], the most robust predictors were identified as those in which the upper boundary of the $95 \%$ CI of the FPR was below $5 \%$.

For some neuroprognostic tests used after cardiac arrest, such as the blood values of biomarkers of neurological injury or the grey matter to white matter density ratio on brain $\mathrm{CT}$, the results are expressed as a continuous variable. In this case, sensitivity and specificity will depend on the value of the variable that is chosen as a threshold to separate positive from negative test results, and the values of sensitivity and specificity that are obtained by varying the positivity threshold across all its possible values are expressed by a receiver operating 
characteristic (ROC) curve. The problem with dichotomising continuous predictive variables to obtain a binary test result is that finding a consistent threshold for $100 \%$ specificity is difficult. Very high values of test results can be caused by outliers, which cause distortion and reduce test sensitivity.

\section{Main sources of bias in neuroprognostication}

One of the major biases in neuroprognostication after cardiac arrest is self-fulfilling prophecy. This occurs when the treating team is aware of the result of the prognostic test and uses it for decisions that affect patient's outcome, e.g. WLST. This leads to an overestimation of the test performance, and-potentially-to an inappropriate WLST. In a systematic review on neuroprognostication after cardiac arrest published in 2013 [298, 299], 64/73 $(88 \%)$ studies were at risk of self-fulfilling prophecy bias.

Ideally, to avoid self-fulfilling prophecy bias, the index tests should be investigated blindly. However, this is difficult to achieve in practice. Concealing results of clinical examination from the treating team is almost impossible, while concealing results of EEG or brain imaging would be unethical, since they may reveal the presence of potentially treatable complications (e.g. seizures or intracranial hypertension, respectively). Nevertheless, some predictors such as biomarkers have been evaluated blindly [230]. A special condition limiting the risk of self-fulfilling prophecy bias is the absence of an active WLST policy. This has been described in some studies conducted in countries or communities where treatment limitations are not accepted due to cultural, legal or religious reasons [300, 301].

Other strategies to reduce the risk of falsely pessimistic predictions include avoiding confounding from treatments (e.g. sedatives or other drugs) affecting the results of some predictors, such as clinical examination or EEG; avoiding basing decisions on life-sustaining treatments on the results of a single index test, but rather using a multimodal approach (see Fig. 4-multimodal prognostication algorithm); and always interpreting the results of the index tests within the clinical context.

A specific source of bias in neuroprognostic studies after cardiac arrest is the presence of a time lag between the recording of the index test, which is usually done very early after the arrest, and the assessment of the target condition, i.e. neurological outcome. Since recovery from hypoxic-ischaemic brain injury following cardiac arrest requires time, the minimum recommended timing for its assessment is 30 days or later from the event or neurological discharge [290]. However, further neurological recovery can occur later. Consequently, an early prediction of outcome which is confirmed by CPC or mRS measured at hospital discharge may occasionally prove false when the outcome is reassessed later [302]. For that reason, guidelines suggest reassessing neurological outcome at 3 or 6 months after the event [295]. The majority of studies included in the systematic review informing the present guidelines reports neurological outcome at least 6 months after cardiac arrest [15].

Another bias which is partly related to the time delay between index test assessment and outcome is the interference from extracerebral causes of death after cardiac arrest. These include cardiovascular instability, which is the second most common cause of in-hospital death after cardiac arrest [23], and multiple organ failure due to global ischaemia-reperfusion injury [303, 304]. Although the incidence of these complications is maximal early after arrest, death from extracerebral organ failure may occur after neurological recovery [305]. The prevalence of death after awakening was $16 \%$ in ICU in a single-centre study [306], and 4.2\% during hospital stay in a recent multicentre European study including 4646 patients [307]. In that study, death occurred at a median time of 9 (3-18) days after awakening, and it was more common after IHCA than after OHCA.

\section{Clinical examination}

These guidelines are supported by evidence derived from a systematic review on prognostication and 2020 ILCOR CoSTRs $[9,15]$. The relevant treatment recommendations in the 2020 ILCOR CoSTR are the following:

- We suggest using pupillary light reflex at $72 \mathrm{~h}$ or later after ROSC for predicting neurological outcome of adults who are comatose after cardiac arrest (weak recommendation, very-low-certainty evidence).

- We suggest using quantitative pupillometry at $72 \mathrm{~h}$ or later after ROSC for predicting neurological outcome of adults who are comatose after cardiac arrest (weak recommendation, low-certainty evidence).

- We suggest using bilateral absence of corneal reflex at $72 \mathrm{~h}$ or later after ROSC for predicting poor neurological outcome in adults who are comatose after cardiac arrest (weak recommendation, very-low-certainty evidence).

- We suggest using presence of myoclonus or status myoclonus within $96 \mathrm{~h}$ after ROSC, in combination with other tests, for predicting poor neurological outcome in adults who are comatose after cardiac arrest (weak recommendation, very-low-certainty evidence). We also suggest recording EEG in the presence of myoclonic jerks in order to characterise the phenotype of the myoclonus. 
Ocular reflexes

Ocular reflexes currently used for neurological prognostication after cardiac arrest include pupillary reflex and corneal reflex. The pupillary light reflex (PLR) comprises a temporary reduction of pupil size induced by a light stimulus. Standard PLR (s-PLR) is evaluated visually and elicited generally using a penlight. In recent years, a quantitative evaluation of PLR using portable pupillometers has become available in the ICU. A bilaterally absent s-PLR has low specificity for predicting poor outcome in the first hours after ROSC, but its accuracy progressively increases, and it achieves $100 \%$ specificity after $96 \mathrm{~h}$ from ROSC with $20-25 \%$ sensitivity [15]. This is presumably due to the process of brain recovery after anoxic-ischaemic injury, but it may be due partly to interference from sedatives used in the early post-resuscitation phase to maintain TTM. Standard PLR is inexpensive and easy to use, but it is subjective and prone to interrater variability [308].

Quantitative evaluation of PLR (automated pupillometry) provides an objective and quantifiable measurement of the pupillary response. The most common pupillometry measures are the percentage reduction of pupillary size, generally indicated as qPLR [309] and the neurological pupil index (NPi) [310]. NPi is calculated from several dynamic parameters of the pupillary response (including constriction and dilation velocity, size and percentage size reduction after stimulation) using a proprietary algorithm. A NPi value $\geq 3$ is considered normal. Limited evidence showed that, unlike s-PLR, NPi can predict unfavourable outcome with no false positive results from $24 \mathrm{~h}$ or less to $72 \mathrm{~h}$ from ROSC [15]. In one study this was because of the ability of pupillometer to detect a response even when the pupil size was very small, presumably from sedation [310]. Results of pupillometry are expressed as a continuous measure, and threshold for $100 \%$ specificity varied among studies. In three studies included in a recent review this threshold for NPi was $<2.4$ before $24 \mathrm{~h}$ and 2.0 at $24-72 \mathrm{~h} \mathrm{[15].} \mathrm{Another} \mathrm{limitation} \mathrm{of} \mathrm{automated}$ pupillometry is its additional cost.

The corneal reflex (CR) is elicited by touching the outer margin (limbus) of the cornea with a cotton wisp. Alternatively, in order to minimise the risk of corneal abrasion, an air or water squirt can be used [311]. The corresponding response is represented by an eye blink. In patients who are comatose after cardiac arrest, an absent CR predicts poor neurological outcome after $72 \mathrm{~h}$ from ROSC with $100 \%$ specificity and $25-40 \%$ sensitivity [15]. Like PLR, CR is prone to interference from sedation. In addition, it may be affected by muscle relaxants. A recent survey showed that the modalities with which $C R$ is assessed in comatose patients are inconsistent [312].

\section{Motor response}

An absent or extensor motor response to pain (motor component [M] 1 or 2 of the Glasgow Coma Score) is associated with poor neurological outcome after cardiac arrest [15]. However, its specificity is low, almost never achieving 100\%, even when it is assessed after $96 \mathrm{~h}$ from ROSC. Like CR, motor response is based on striate muscle contraction and, as such, it can be affected by muscle relaxants. Because of its high sensitivity $(>60 \%$ at $72 \mathrm{~h}$ or later from ROSC) a $M=1-2$ can be used as a criterion for identifying patients needing prognostication after cardiac arrest. However, recent evidence showed that using $M \leq 3$ as an entry point increases the sensitivity for prediction of poor outcome without reducing specificity [313].

\section{Myoclonus and status myoclonus}

Myoclonus consists of sudden, brief, involuntary jerks caused by muscular contractions or inhibitions. Their distribution can be focal, multifocal or generalised [314]. Presence of myoclonus within $96 \mathrm{~h}$ from ROSC in patients who are comatose after cardiac arrest is associated with poor neurological outcome in most cases [15]. However, a false positive rate of up to $22 \%$ has been described [315]. Most prognostication studies did not provide a definition or description of myoclonus. In some patients with favourable outcome, myoclonus may persist after recovery of consciousness in a chronic form of action myoclonus (i.e. triggered by spontaneous movements) known as Lance-Adams syndrome $[182,316]$.

Clinical myoclonus can inconsistently be associated with electrical seizures; therefore, recording an EEG can be useful. Some studies have identified specific EEG features associated with benign myoclonus, such as a reactive $[179,184]$ and/or continuous EEG background [179, 181]. The presence of diffuse and continuous myoclonic jerks is usually described as status myoclonus. However, a consensus definition of status myoclonus is lacking. The 2014 ERC-ESICM Advisory Statement on neurologic prognostication after cardiac arrest suggested that in comatose survivors of cardiac arrest status myoclonus should be defined as a continuous and generalised myoclonus persisting for $30 \mathrm{~min}$ or more [297]. Evidence from two studies that did not distinguish electrographic features of status myoclonus [15] showed that status myoclonus within $24 \mathrm{~h}$ [317] or within 7 days from ROSC $[178,317]$ is almost invariably associated with poor neurological outcome (specificity $99-100 \%$ ).

The advantages of predictors based on clinical examination include minimal equipment and costs (except pupillometry) and availability at the bedside. Their major limitations include interference from sedatives, opioids, and-except for the PLR-from muscle relaxants. In 
addition, their assessment is prone to subjectivity. Use of automated assessment, like pupillometry for PLR, may at least address these limitations. Finally, results of clinical examination cannot be concealed from the treating team, potentially causing a self-fulfilling prophecy bias.

\section{Neurophysiology}

These guidelines are supported by evidence derived from a systematic review on prognostication and 2020 ILCOR CoSTRs $[9,15]$. The relevant treatment recommendations in the 2020 ILCOR CoSTR are:

- We recommend that neuroprognostication always be undertaken using a multi-modal approach because no single test has sufficient specificity to eliminate false positives (strong recommendation, very-lowcertainty evidence).

- We suggest using a bilaterally absent N20 wave of somatosensory evoked potential (SSEP) at $\geq 24 \mathrm{~h}$ from ROSC in combination with other indices to predict poor outcome in adult patients who are comatose after cardiac arrest (weak recommendation, very-low-certainty evidence).

- We suggest against using the absence of EEG background reactivity alone to predict poor outcome in adult patients who are comatose after cardiac arrest (weak recommendation, very-low-certainty evidence).

- We suggest using the presence of seizure activity on EEG in combination with other indices to predict poor outcome in adult patients who are comatose after cardiac arrest (weak recommendation, very-low certainty evidence).

- We suggest using burst-suppression on EEG at $\geq 24 \mathrm{~h}$ from ROSC in combination with other indices to predict poor outcome in adult patients who are comatose and who are off sedation after cardiac arrest (weak recommendation, very-low-certainty evidence).

\section{Electroencephalography (EEG)}

Electroencephalography (EEG) is one of the most widely used and studied methods to assess brain function and prognosis after cardiac arrest [318]. EEG is also important for diagnosing and treating seizures.

The main aspects when assessing EEG are the background activity, superimposed discharges and reactivity. The EEG background continuity is most important for the prognosis and is commonly categorised as continuous, discontinuous, burst suppression (50-99\% suppression periods) or suppression ( $>99 \%$ activity $<10 \mu \mathrm{V}$ amplitude) [319]. A standardised terminology for critical care EEG has been proposed by the ACNS [187].
Immediately after a cardiac arrest, the EEG is suppressed in many patients, but it returns to a continuous normal voltage EEG within the first $24 \mathrm{~h}$ in most patients who ultimately achieve a good outcome [320, 321]. The time for this restitution is correlated with outcome [319, 322]. The EEG-background is often discontinuous and of low frequency on its first appearance [320,323]. Sedative drugs affect background continuity and have the potential to induce discontinuous or burst-suppression background in a dose-dependent manner [324, 325].

\section{Background pattern: Suppression}

A suppressed $(<10 \mu \mathrm{V})$ or low-voltage $(<20 \mu \mathrm{V})$ background is relatively common during the first day after a cardiac arrest in patients who later recover [300, 320, 321]. However, $24 \mathrm{~h}$ after ROSC, a suppressed EEGbackground $<10 \mu \mathrm{V}$ is a reliable sign of a poor prognosis [326-331] although two false positive predictions by this pattern 48-72 $\mathrm{h}$ after cardiac arrest were reported in one study [328]. There was moderate interrater agreement for suppressed background among senior neurophysiologists $[328,332]$.

\section{Background pattern: Burst suppression}

According to the ACNS-terminology, burst suppression (BS) is defined as $50-99 \%$ of the recording consisting of suppression alternating with bursts. The terminology does not have any amplitude criteria for the bursts but these may be defined further as 'highly epileptiform bursts', based on appearance [187]. 'Presence of 'identical bursts' (either the first $0.5 \mathrm{~s}$ of each burst or each stereotyped cluster of $\geq 2$ bursts appears visually similar in $>90 \%$ of bursts in each channel) portend a poor prognosis in post-anoxic coma [333]. One research group also proposed a separation of BS-patterns into 'synchronous' (with highly epileptiform or identical bursts) and 'heterogenous' (not 'synchronous') [331]. The criteria used for burst amplitude and appearance varies considerably between studies. A substantial portion of patients with BS during the first $24 \mathrm{~h}$ and occasional patients with BSpattern after $24 \mathrm{~h}$ still have a good outcome, which is possibly related to sedation use [302, 320, 326-328, 334336]. There was substantial interrater agreement among experienced neurophysiologists for BS [328].

\section{Background pattern: Discontinuous}

A discontinuous background with suppression periods $>10 \%$ of the recording has low prognostic performance during the first $24 \mathrm{~h}$ after cardiac arrest [337, 338] and an inconsistent performance after $24 \mathrm{~h}$ [326-328, 338]. 
Background pattern: Reactivity

EEG-reactivity is a measurable change in amplitude or frequency upon external stimulation (auditory and pain). There is no generally acknowledged standard for reactivity testing and the prognostic performance of this feature varied substantially between studies $[15,339]$. Absence of EEG-reactivity during the first $24 \mathrm{~h}$ after cardiac arrest is an indicator of a poor outcome with high sensitivity but low specificity $(41.7-87.5 \%)$ [336, 340-342]. After $24 \mathrm{~h}$, the sensitivity of absent reactivity remains high but the specificity varied from 50 to $100 \%$ [326, 328, 334, 336, 341-345]. Interrater agreement for the assessment of EEG-reactivity varied from slight-almost perfect [328, 346]. Stimulusevoked rhythmic, periodic or ictal discharges (SIRPIDS) are not a manifestation of normal background reactivitytheir prognostic significance is still undefined [203, 347].

\section{Superimposed patterns: Periodic discharges}

A 'periodic' pattern is a waveform that occurs repeatedly, with a quantifiable interval between discharges. If no such interval exists, the pattern is termed 'rhythmic' [187]. Periodic discharges (PDs) may be superimposed on various backgrounds and are related to a worse prognosis. Generalised periodic discharges (GPDs) are a sign of a poor prognosis with limited specificity [326, 327, 330, 334]. In general, the background on which PDs appear is more related to the neurological outcome [319]. PDs on a continuous and reactive EEG-background should not be considered as an indicator of a poor outcome [181].

Superimposed patterns: Sporadic epileptiform discharges 'Sporadic epileptiform discharges' describes sharp waves or spikes resembling those seen in patients with epilepsy, but without the regularity of a periodic pattern. The frequency by which they appear may vary extensively from 'rare' $(<1 / \mathrm{h})$ to 'abundant' $(\geq 1 / 10 \mathrm{~s})$, bordering on periodic discharges. While their appearance is related to a worse outcome, their specificity to predict poor outcome ranges from 66.7 to $100 \%$ [15] and reports on the potentially important frequency or number of discharges was lacking in studies [300, 328, 330, 331]. Presence of sporadic epileptiform discharges is NOT a reliable indicator of a poor neurological prognosis.

Sporadic epileptiform discharges: Electrographic seizures and electrographic status epilecticus

The ACNS defines 'unequivocal seizures' as generalised rhythmic spike-and-wave discharges with a frequency $\geq 3 \mathrm{~Hz}$ or clearly evolving discharges of any type $>4 \mathrm{~Hz}$ [187]. This definition was inconsistently used in studies. Seizures had a low sensitivity but high specificity for a poor outcome regardless of timing [326, 328, $330,334,348]$.
The term 'electrographic status epilepticus' (ESE) is defined as an electrographic seizure for $\geq 10$ continuous minutes or for a total duration of $\geq 20 \%$ of any 60 - $\mathrm{min}$ period of recording. This definition has been included for the first time in the 2021 update of the ACNS terminology and none of the currently available prognostic studies has incorporated it yet. Some studies based their definition of ESE on the ACNS classification of unequivocal seizures extending $\geq 30 \mathrm{~min}$ but also included epileptiform discharges $\geq 1 \mathrm{~Hz}[197,322]$, and in one study $\geq 0.5 \mathrm{~Hz}$ as ESE [349]. Other studies had an unclear definition of ESE [302, 334, 335, 341]. The proportion of patients classified with ESE varied considerably between studies, possibly reflecting differences in definitions. One study showed that ESE evolves from high frequency discharges early after onset to progressively slower frequencies during the following days and weeks [186]. Regardless of the classification used, ESE is associated with a worse prognosis after cardiac arrest, but some patients have a good outcome [196, 197, 199]. As with periodic discharges, it is important to consider if the EEG-background is continuous and reactivity is present, which are both favourable features [197, 199]. Because of the lack of a standardised classification, we recommend avoiding the term 'status epilepticus' for assessments of prognosis but instead to classify the EEG-background and superimposed discharges and unequivocal seizures according to the standardised ACNS terminology [187].

\section{Categories of patterns}

In several studies, the most unfavourable patterns were grouped as 'malignant' or 'highly malignant.' The most common grouping included suppressed background with or without periodic discharges and burst-suppression as 'highly malignant patterns' [326]. There was substantial interrater agreement for these 'highly malignant patterns' [346] and the specificity for poor outcome was 90.6$100 \%$ [326, 327, 329, 336, 338, 340, 350]. An alternative categorisation of 'unfavourable patterns' using a stricter definition of burst-suppression has been suggested [331].

\section{Quantitative EEG-indices}

Automated assessment of quantitative EEG-features such as the burst-suppression amplitude ratio and reactivity has been tested in individual studies [351, 352]. Combinations of quantitative EEG-features include the Bi-spectral index (BIS) and the Cerebral Recovery Index [353]. The threshold value and specificity for BIS to predict poor outcome varied widely between studies [354-356]. Automated assessments may decrease subjectivity in EEG assessments. Prospective multi-centre studies are needed to assess the prognostic performance after cardiac arrest. 
Evoked potentials: Somatosensory evoked potentials (SSEPs)

When performing SSEPs the median nerve is electrically stimulated and the ascending signals are recorded from the peripheral plexus brachialis, cervical level, subcortical level and the sensory cortex (N20-potential). SSEPs may be depressed by barbiturate coma but are preserved with other sedative drugs such as propofol and midazolam [357]. A bilateral absence of the short-latency N20-potentials over the sensory cortex is a reliable sign of a poor prognosis after cardiac arrest with high specificity and limited sensitivity both early and late after cardiac arrest [201, 202, 302, 310, 331, 335, 337, 338, 340, $342,343,350,352,358-366]$. Occasional false positive predictions were reported [367]. The interrater reliability for interpretation of SSEPs was moderate to good [368, 369]. The quality of the recording is very important and noise from muscle activity is an important limiting factor which may be eliminated by neuromuscular blocking drugs $[357,368]$.

Evoked potentials: Visual evoked potentials (VEP) and auditory evoked potentials (AEP)

There are few data supporting the use of visual evoked potentials (VEPs) [358] and auditory evoked potentials (AEPs) $[361,364]$ to prognosticate outcome after cardiac arrest. These results need validation before VEPs or AEPs can be recommended in this context.

\section{Biomarkers}

These guidelines are supported by evidence derived from a systematic review on prognostication and 2020 ILCOR CoSTRs $[9,15]$. The relevant treatment recommendations in the 2020 ILCOR CoSTR are as follows:

- We recommend that neuroprognostication always be undertaken using a multi-modal approach because no single test has sufficient specificity to eliminate false positives (strong recommendation, very-lowcertainty evidence).

- We suggest using neuron-specific enolase (NSE) within $72 \mathrm{~h}$ after ROSC, in combination with other tests, for predicting neurological outcome of adults who are comatose after cardiac arrest (weak recommendation, very-low-certainty evidence). There is no consensus on a threshold value.

- We suggest against using S-100B protein for predicting neurological outcome of adults who are comatose after cardiac arrest (weak recommendation, low-certainty evidence).

- We suggest against using serum levels of glial fibrillary acidic protein (GFAP), serum tau protein, or neurofilament light chain (Nfl) for predicting poor neurological outcome of adults who are comatose after cardiac arrest (weak recommendation, verylow-certainty evidence).

Protein biomarkers that are released following injury to neurons and glial cells may be measured in blood and are likely to correlate with the extent of brain injury and with neurological outcome. Neuron-specific biomarkers include NSE, Nfl and tau protein, while S100B and GFAP originate from astrocytes. Neuron specific enolase has been recommended for assessment of brain injury and to help prognosticate outcome after cardiac arrest since the last revision in 2015 [2]. Their actual use in clinical practice, however, is not known. Several reports on novel biomarker candidates have been published since 2015 [231, 370-372].

Importantly, a multimodal approach should be used for assessment of comatose survivors after cardiac arrest. Advantages of biomarkers include quantitative results, the relative ease of sampling and interpretation and their independence from the effects of sedatives. Limitations include availability, lack of robust laboratory references, insufficiently large study populations and lack of external validation for some. Most of the available evidence is limited to a time span of up to $72 \mathrm{~h}$ after cardiac arrest which is relevant for most patients. However, it necessitates a strategy for prospective sampling before the assessment of prognosis $>72 \mathrm{~h}$ post-arrest. Very limited evidence supports the use of biomarkers after $72 \mathrm{~h}$ in patients who fail to awaken. Large studies investigating and validating promising novel biomarkers are needed to confirm their predictive value, to assess their reproducibility and to identify consistent thresholds for a specificity that should be close to $100 \%$. The rationale for accepting a specificity of less than $100 \%$ would be that using blood biomarkers, there will always be outliers that must be taken into consideration, e.g. due to poor calibration or issues with laboratory standards, haemolysis or due to poor technique in handling of samples. Demanding $100 \%$ specificity from a blood biomarker will lower the sensitivity to levels where their clinical use can be questioned, while allowing an FPR of $1 \%$ or $2 \%$ will increase their clinical relevance. With a multimodal approach, every prognostic method used for assessment of an individual patient must point in the same direction in order to be trusted. This may be particularly true for biomarkers because of their continuous nature; normal or mildly elevated levels (at correct sampling time) should always alert the clinician of potential error in other methods indicating poor prognosis. 
Neuron-specific enolase (NSE)

Neuron-specific enolase has been studied extensively; since the last systematic reviews [298, 299], at least 13 observational studies have been published with threshold values ranging from 33 to $120 \mu \mathrm{g} \mathrm{L}{ }^{-1}$ within $72 \mathrm{~h}$ predicting poor neurological outcome from hospital discharge to 6 months with specificity ranging from 75 to $100 \%$ and sensitivity ranging from 7.8 to $83.6 \%$. In the largest study to date, outliers were described [373]. Patients with high NSE $\left(>90 \mu \mathrm{g} \mathrm{L}^{-1}\right)$ and good outcome had confounders for NSE elevation and most patients with low NSE $(<17 \mu \mathrm{g}$ $\mathrm{L}^{-1}$ ) who died had a cause of death other than hypoxic/ ischaemic encephalopathy. The study was excluded from the recent systematic review because the primary outcome was CPC at ICU discharge [15]. A large substudy of the TTM-trial identified a threshold of $48 \mu \mathrm{g} \mathrm{L}{ }^{-1}$ at $48 \mathrm{~h}$ and a threshold of $38 \mu \mathrm{g} \mathrm{L}{ }^{-1}$ at $72 \mathrm{~h}$ with a specificity of 98\% (FPR 2\%) for poor neurological outcome at 6 months [230]. In another study, NSE with a threshold of $50.2 \mu \mathrm{g}$ $\mathrm{L}^{-1}$ at day 4 predicted poor neurological outcome at 1 month with $100 \%$ specificity and $42.1 \%$ sensitivity [374].

NSE decreases after $24 \mathrm{~h}$ in patients with good outcome and typically increases in patients with a poor outcome to peak at $48-96 \mathrm{~h}$. NSE performs poorly at $24 \mathrm{~h}$ and best at 48 or $72 \mathrm{~h}$. High NSE at 48 or $72 \mathrm{~h}$ after cardiac arrest is a robust predictor of a poor outcome [230, 365, 373-378]. Increasing NSE from 24 to 48 or $48-72 \mathrm{~h}$ is a reliable indicator of a poor prognosis with similar performance as the absolute value [379]. One small study found that a $48: 24$ h NSE ratio $\geq 1.7$ had a $100 \%$ specificity for poor outcome [375]. In a recent study, the prognostic performance of NSE was clearly dependent on age and severity of the insult (time to ROSC) [380]. It performed best in the youngest quartile and in patients with longer time to ROSC. Several different analytical assays were used in the included studies but the methodology for routine clinical use provided by Roche and Brahms was most frequent. NSE has been used as a surrogate marker for brain injury in two recent trials $[75,96]$.

Thresholds for high NSE values must be established in collaboration with the local laboratory considering the analytical method. Red blood cells contain NSE so haemolysis (free haemoglobin) must be measured and samples discarded if the haemolysis index threshold is exceeded because this may generate a falsely high NSE value [381]. The half-life of free haemoglobin is approximately $2-4$ h compared with the 30-h half-life of NSE. Thus, the NSE value may be inappropriately increased (by NSE from red blood cells) at a time when free haemoglobin is no longer detectable, which is a concern when using NSE for prognostication after cardiac arrest [381].
S100B

Three observational studies have been published since 2013 $[376,377,382]$, two of them investigated S100B immediately after ROSC and identified threshold values ranging from 3.56 to 16.6 with $100 \%$ specificity of poor outcome but with low sensitivities of $2.8 \%$ to $26.9 \%$. In the largest study, S100B discriminated best at $24 \mathrm{~h}$ with a threshold value of 2.59 $\mu \mathrm{g} \mathrm{L} \mathrm{L}^{-1}$ for $100 \%$ specificity but with a low sensitivity of $10 \%$, the corresponding sensitivity for $98 \%$ specificity ( $2 \%$ FPR) was $32 \%$ (threshold value $0.36 \mu \mathrm{g} \mathrm{L}^{-1}$ ) [382]. The authors concluded that $\mathrm{S100B}$ did not add any real value to present prognostication models with or without NSE. S100B is also very rarely used in clinical practice and for these reasons is not included in our recommendations.

\section{Glial fibrillary acidic protein (GFAP)}

In one observational study with 100 patients, GFAP with a threshold value of $0.08 \mu \mathrm{g} \mathrm{L}{ }^{-1}$ at $48 \mathrm{~h} \pm 12 \mathrm{~h}$ predicted poor neurological outcome at 1 month with $100 \%$ specificity and $21.3 \%$ sensitivity [370].

\section{Serum tau}

In one study, serum tau protein with a threshold value ranging from 72.7 to $875.6 \mathrm{ng} \mathrm{L}^{-1}$ predicted poor neurological outcome at 6 months with $100 \%$ specificity and a sensitivity of $4-42 \%$ (very-low certainty of evidence) [371]. An ultra-sensitive single molecule assay (SIMoA) was used, with a detection limit at the single molecular level [383].

\section{Serum neurofilament light chain (Nfl)}

In one large study, serum $\mathrm{Nfl}$ with a threshold value ranging from 1539 to $12,317 \mathrm{pg} \mathrm{mL}^{-1}$ at $24-72 \mathrm{~h}$ predicted poor neurological outcome (CPC 3-5) at 6 months with $100 \%$ specificity and sensitivity ranging from 53.1 to $65 \%$ (moderate certainty of evidence) [231]. The same ultrasensitive SIMoA technique was used for detection of $\mathrm{Nfl}$ as was used for tau protein (see above). In a post-hoc analysis of the COMACARE trial, which used the same SIMoA technique for analysis, thresholds for serum $\mathrm{Nfl}$ that achieved $99 \%$ specificity for a poor outcome were 127,262 and $344 \mathrm{pg} \mathrm{mL}^{-1}$ at $24 \mathrm{~h}, 48 \mathrm{~h}$ and $72 \mathrm{~h}$, respectively; sensitivities ranged from 78 to $85 \%$ [384]. In another study that did not use the SIMoA technique, serum Nfl with a threshold value ranging from 252 to $405 \mathrm{pg} \mathrm{mL}^{-1}$ from day 1 to day 7 predicted poor neurological outcome (CPC 4-5) at 6 months with $100 \%$ specificity and sensitivity ranging from 55.6 to $94.4 \%$ [372].

\section{Imaging}

These guidelines are supported by evidence derived from a systematic review on prognostication and 2020 ILCOR 
CoSTRs [9, 15]. The relevant treatment recommendations in the 2020 ILCOR CoSTR are:

- We suggest using brain imaging studies for prognostication only in centres where specific experience is available (weak recommendation, very-low-quality evidence).

- We suggest using the presence of a marked reduction of the grey matter/white matter (GM/WM) ratio on brain CT within $72 \mathrm{~h}$ after ROSC or the presence of extensive diffusion restriction on brain MRI at 2-7 days after ROSC in combination with other predictors for prognosticating a poor neurologic outcome in patients who are comatose after cardiac arrest and who are treated with TTM (weak recommendation, very-low-quality evidence).

\section{Computed tomography (CT) of the brain}

Following cardiac arrest, hypoxic-ischaemic brain injury causes cytotoxic oedema, which appears as an attenuation of the GM/WM interface, and vasogenic oedema leading to brain swelling, visible as an effacement of cortical sulci [385]. Measurement of the ratio between the GM and the WM densities (GWR), expressed in Hounsfield units is a method to quantify the degree of oedema. The density of the GM is higher than that of the WM, so that GWR is normally higher than 1 . The lower the GWR, the greater the severity of brain oedema.

GWR reduction occurs early in patients with severe hypoxic-ischaemic brain injury. In a recent systematic review, most studies on reduced GWR showed that this sign was $100 \%$ specific for poor neurological outcome as early as $1 \mathrm{~h}$ after ROSC [15]. However, in other studies [301, 386-388], a reduced GWR was 100\% specific for poor neurological outcome up to $72 \mathrm{~h}$ after ROSC. The methods for GWR measurement varied across studies. In most of them, GWR was calculated between GM and WM areas within the basal ganglia. In others, measurements within the cerebrum (centrum semiovale and high convexity area) were performed [389-391]. In almost all studies, a GWR threshold for $100 \%$ specificity was identified. However, its value varied across studies. For instance, the threshold for $100 \%$ specificity of the average GWR measured at the basal ganglia and the cerebrum ranged between 1.1 and 1.23 within 2 h from ROSC [15]. GWR sensitivity also varied widely across studies, probably reflecting differences between scanners and software [392], in the methods of calculation, or in the aetiology of the arrest [390, 393]. In one substudy of the TTM trial, oedema on brain CT was assessed visually without formal GWR measurement [394]. In that study, specificity for poor neurological outcome was 98.4 (94.3-99.6)\% with 33.6 (28.1-39.5)\% sensitivity. Most studies on brain CT were single centre with retrospective design.

\section{Magnetic resonance imaging (MRI) of the brain}

Along with $\mathrm{CT}$, magnetic resonance imaging (MRI) of the brain is the most investigated imaging-based predictive index in patients who are comatose after cardiac arrest [15]. Brain MRI is more challenging to perform in ventilated ICU-patients and MRI was generally performed later than brain CT, usually at $48 \mathrm{~h}$ or later from ROSC. On brain MRI, cytotoxic oedema from hypoxic-ischaemic brain injury appears as a hyperintensity on diffusionweighted imaging (DWI) sequences [395]. In several studies, presence of DWI lesions is associated with poor neurological outcome after cardiac arrest [389, 396-399]. However, the assessment was done qualitatively, and specificity was inconsistent (range 55.7-100\%). Apparent diffusion coefficient (ADC) enables a semiquantitative assessment of DWI changes, therefore limiting subjectivity. However, the ADC metrics in prognostication studies varied [15]. These include lowest minimum or mean ADC [400], mean ADC [401], the proportion of brain volume below a given ADC threshold [401, 402] and the maximum size of the MRI clusters with minimum ADC [400]. Most of these studies assessed global ADC, while one of them assessed regional ADC [400]. In all these studies, an ADC threshold for $100 \%$ specificity was identified, often with sensitivities above $50 \%$. All studies on ADC MRI had a small sample size, which limited their precision. In many studies, imaging was performed at the discretion of the treating physician, which may have introduced a selection bias.

Unlike clinical examination and EEG, imaging studies are not prone to interference from sedative drugs. In addition, they can be assessed blindly. Their major limitation is the lack of standardisation of measurement techniques. Despite the available studies showed a high accuracy both for brain CT and MRI, the number of studies was limited with a wide variability in the adopted measurement techniques which greatly limits the reproducibility of their results. For this reason, it is reasonable to reserve the use of imaging studies for prognostication only in centres where specific experience is available. Since there is currently no standard for CTGWR or MR-ADC measurements these techniques can be recommended to confirm the presence of generalised and extensive ischemic injury apparent from conventional visual analysis by an experienced neuroradiologist. Finally, imaging studies cannot be performed at the bedside and MRI may not be feasible in the most unstable patients, which limits its applicability especially in the early post-resuscitation period. 


\section{Multimodal prognostication}

In 2015, the ERC-ESICM Guidelines on Post-Resuscitation Care included an algorithm for the prediction of poor neurological outcome in patients who are comatose after cardiac arrest [1]. This algorithm has been validated in recent retrospective studies. One study in 226 patients showed that the 2015 ERC/ESICM prognostication guidelines had a $0 \%$ FPR for predicting poor outcome (CPC from 3 to 5) both at hospital discharge and at 6 months [302]. Similarly, in a larger single-centre cohort including 485 comatose resuscitated patients the ERC-ESICM algorithm predicted CPC $3-5$ with $0 \%$ FPR in 155 patients [403]. Among the remaining 330 patients in whom no major predictor or combination of predictors suggesting poor outcome were detected, two thirds had good neurological outcome at 3 months. Finally, in a retrospective multicentre cohort of 585 patients from the TTM trial, the ERC-ESICM algorithm had 0\% (95\% CI $0-1.2 \%)$ FPR for predicting poor neurological outcome at 6 months [313].

The 2015 ERC-ESICM prognostication algorithm was based on a combination of predictors including results of clinical examination (absent or extensor motor response, absent pupillary and corneal reflexes, status myoclonus), biomarkers (high blood values of NSE), electrophysiology (unreactive burst-suppression or status epilepticus on EEG, bilaterally absent N20 SSEP wave) and imaging (signs of diffuse anoxic brain injury on CT or MRI). The evidence supporting these predictors had been assessed in two reviews published in 2013 [298, 299]. In order to provide an update for the present guidelines, a new review has been conducted and its results are reported in the previous paragraphs of the present guidelines focusing on individual prognostication modalities [15]. The 2020 review largely confirmed the results of the 2013 reviews and the reliability of the predictors suggested in the 2015 algorithm. However, some important differences were noted:

- Absent pupillary and corneal reflexes achieved $0 \%$ FPR consistently only after day 4, rather than after day 3 as in the previous review

- Automated measurement of absent pupillary reflex using pupillometry may enable a more accurate prediction than standard (manual) assessment of pupillary reflex (s-PLR), and it is more reproducible

- The accuracy of NSE was higher at 48-72 $\mathrm{h}$ than at $24 \mathrm{~h}$ from ROSC

- The low FPR of unreactive EEG background documented in a few of the studies on TTM-treated patients in the 2013 review was not confirmed in the 2020 review
- No consistent definition was found for status epilepticus, a predictor suggested in the 2015 guidelines

- Presence of a suppressed EEG background or burstsuppression predicted poor outcome with very-low FPR, especially when recorded after 24-72 h from ROSC; in the previous reviews, evidence supporting suppression was negligible, and definitions of burstsuppression were heterogeneous

- Several prognostication studies classified EEG according to the Standardized Critical Care EEG Terminology (2012 version) of the American Clinical Neurophysiology Society (ACNS) [404].

The risk of bias for most of the available studies was high. As in previous reviews, a major limitation in most studies was lack of blinding; furthermore, several predictors of poor neurological outcome were used as criteria for WLST. In both cases, this may have resulted in a selffulfilling prophecy. However, the 2020 review included studies where no WLST was performed, therefore limiting the risk of self-fulfilling prophecy [300, 358, 387, 393, 398]. Predictors assessed in these studies included EEG, SSEPs and brain CT. Based on results of the 2020 review, most of the recommendations included in the 2015 prognostication algorithm remain valid.

\section{Suggested prognostication strategy (Fig. 4)}

Prognostic assessment should start with an accurate clinical examination [405]. Its main scope is to confirm that the patient is comatose because of hypoxic-ischaemic brain injury. Clinical examination should be performed daily to detect signs of neurological recovery such as purposeful movements or to identify a clinical picture suggesting impending brain death. The latter may include fixed, dilated pupils, diabetes insipidus and cardiovascular changes suggesting herniation, such as bradycardia associated with hypertension or an otherwise unexplained haemodynamic instability. Brain death occurs in $5-10 \%$ of patients who die after cardiac arrest resuscitated with conventional CPR and in about $25 \%$ of patients who die after resuscitation with extracorporeal CPR [286]. In most cases, brain death occurs during the first 3-4 days after ROSC. A suggested algorithm for brain death screening after cardiac arrest is shown in Fig. 6. The World Brain Death Project (WBDP) consensus group has published detailed guidance on the determination of brain death after treatment with targeted temperature management (TTM) [406].

In most patients, awakening from coma following cardiac arrest occurs within 3-4 days from ROSC [202, 305]. However, patients who are initially unconscious following cardiac arrest are usually treated with sedatives and neuromuscular blocking drugs to enable targeted 
temperature management (TTM), and to facilitate mechanical ventilation and other life support measures. Therefore, to enable a reliable clinical examination, these drugs should be stopped for sufficient time to avoid interference from their effects. The WBDP consensus group recommends that clinical examination be delayed until at least five elimination half-lives of the drug administered with the longest half-life [406]. Although this recommendation has been made in the context of diagnosing brain death, it is equally relevant to prognostic assessment if this is being used to make a WLST decision. Short-acting drugs are preferred whenever possible but even a shortacting drug such as propofol has a half-life of 2.3-4.7 h, which implies the need to stop sedatives for at least $24 \mathrm{~h}$ in most cases. This will be much longer if there is renal and/or hepatic impairment or if longer acting drugs have been given. When residual sedation or paralysis is suspected, consider using antidotes to reverse the effects of these drugs. Use caution when administering flumazenil to reverse the effect of benzodiazepines because this may precipitate seizures. Apart from sedation and neuromuscular blockade, other major confounders include hypothermia, severe hypotension, sepsis and metabolic or respiratory derangements.

A poor motor response has a relatively low specificity, but a high sensitivity for prediction of poor neurological outcome after cardiac arrest. Therefore, it can be used to identify patients needing prognostication. An absent or extensor motor response $(M \leq 2)$ of the Glasgow Coma Scale was the entry point of the 2015 prognostication algorithm. However, recent evidence showed that using $M \leq 3$ as an entry point increases the sensitivity for prediction of poor outcome without reducing specificity [313, 407]. The prognostication strategy described below applies to patients who are comatose with a motor response $(M)$ equal to or below 3 (abnormal flexion, extension, or nil) at $\geq 72$ after ROSC. Results of earlier prognostic tests are also considered at this time.

Signs suggesting the potential for recovery should be actively sought. These are often identified early in the clinical course after resuscitation. In a study on 357 comatose survivors of cardiac arrest [338], a benign EEG (continuous, reactive, non-suppressed background without epileptiform discharges) recorded within $24 \mathrm{~h}$ from ROSC predicted good neurological outcome with 76 [69-82]\% sensitivity and 88 [82-92]\% specificity. In 250 patients with indeterminate outcome on day 3 according to the 2015 ERC-ESICM prognostication algorithm presence of a benign EEG was associated with good neurological outcome in 184 cases (positive predictive value 74\%) [403]. Among 14 patients who recovered after their outcome was defined as indeterminate in another validation study, the majority had low and decreasing NSE values and all but one had ventricular fibrillation on the initial ECG [313]. Other potentially useful indices of good neurological outcome include absence of diffusion changes on brain MRI and low blood values of neurofilament light chain within $72 \mathrm{~h}$ from ROSC [231, 389, 397, 398]. Recent evidence showed that a benign EEG is not associated with the presence of other predictors of poor neurological outcome, especially a bilaterally absent N20 SSEP wave [408-410]. Therefore, when predictors suggesting a potential for recovery coexist with others suggesting a poor outcome, there is a chance that the latter signal is a false positive. We suggest that in this case the results of predictive indices are reassessed, and index tests be repeated if possible.

In a comatose patient with $M \leq 3$ at $\geq 72 \mathrm{~h}$ from ROSC, in absence of confounders, poor outcome is likely when two or more of the following predictors are present: no pupillary and corneal reflexes at $\geq 72 \mathrm{~h}$, bilaterally absent N20 SSEP wave at $\geq 24 \mathrm{~h}$, highly malignant EEG at $>24 \mathrm{~h}, \mathrm{NSE}>60 \mu \mathrm{g} \mathrm{L}{ }^{-1}$ at $48 \mathrm{~h}$ and/or $72 \mathrm{~h}$, status myoclonus $\leq 72 \mathrm{~h}$, or a diffuse and extensive anoxic injury on brain CT/MRI. Most of these signs can be recorded before $72 \mathrm{~h}$ from ROSC, however their results will be evaluated only at the time of clinical prognostic assessment. A recent study has shown that a strategy of using $\geq 2$ predictors had 0 [0-8] \% FPR compared with 7 [1-18] \% of the 2015 ERC-ESICM stepwise strategy (due to false positives for pupillary light reflexes) [411].

Evidence from both the 2013 and the 2020 reviews showed that a bilaterally absent N20 SSEP wave is the most widely documented predictor of poor outcome and the one most consistently associated with $100 \%$ specificity. However, false positive predictions have occasionally been reported. In some of these cases, the cause of a false positive result was an incorrect reading of the SSEP record because of artefacts [412]. Neuromuscular blockade improves readability of SSEPs and it should be considered whenever possible [413].

Pupillary light reflex and corneal reflex are also very specific for poor outcome when bilaterally absent at $72 \mathrm{~h}$ or more after ROSC. Based on expert opinion, we suggest that both reflexes should be absent at the time of prognostic assessment for them to reliably predict poor outcome. Unlike SSEPs, ocular reflexes are prone to interference from sedation. Corneal reflexes may also be affected by neuromuscular blocking drugs. These confounders should be excluded before ocular reflexes are assessed. Visual evaluation of PLR may be hampered when the pupil size is less than $6 \mathrm{~mm}$ [308]. Limited evidence shows that in resuscitated comatose patients automated pupillometry is more sensitive than s-PLR in detecting pupil response to light when pupil size is small, which reduces the risk of false positive results [310]. 
Unlike s-PLR, automated pupillometry delivers a stimulating light source with standard characteristics (intensity, duration, and distance from the eye) and measures pupillary response quantitatively, which ensures reproducibility. For this reason, we suggest detecting the absence of PLR with a pupillometer, if available.

Status myoclonus is a prolonged period of myoclonic jerks. Although there is no universal definition for status myoclonus, based on our previous definition [1] we suggest that, in comatose survivors of cardiac arrest, status myoclonus should be defined as a continuous and generalised myoclonus persisting for $30 \mathrm{~min}$ or more. In the 2020 review informing the present guidelines, status myoclonus was documented in two studies, one of which used a definition comparable to that given above. In total, among 113 patients showing this sign, there was only one false positive result. Aside from duration and continuity, other clinical features of myoclonus suggest poor outcome. These include a generalised (vs. focal), axial (vs. distal), or stereotyped (vs. variable) distribution. Conversely, some EEG features, such as a continuous or reactive background or presence of spike-wave discharges synchronised with the myoclonic jerks indicate a potential for good outcome [181]. We suggest recording an EEG in patients with post-arrest status myoclonus, in order both to identify an associated epileptiform activity and to detect signs associated with potential recovery.

Among unfavourable EEG patterns, those more consistently associated with poor neurological outcome are suppression and burst suppression. According to the ACNS, a suppressed EEG background is defined as $>99 \%$ of activity having a voltage less than $10 \mu \mathrm{V}$, while burstsuppression is defined as $50-99 \%$ of the record consisting of suppression, alternated with bursts. In the 2013 reviews, definitions of these patterns were inconsistent. We suggest using the ACNS terminology when assessing these patterns for prognostication, in order to ensure an unequivocal identification [187]. During the first 12-24 h after ROSC, both these patterns have a greater prevalence, but also a higher risk of false positive prediction. Confounding from sedatives used to facilitate TTM may contribute to this. We suggest using these EEG patterns for prognostication only after $24 \mathrm{~h}$ from ROSC. Absence of EEG background reactivity has an inconsistent specificity for poor neurological outcome and we no longer recommend using it for this purpose.

High blood NSE values are a sign of neuronal cell damage and have long been recommended as a predictor of poor neurological outcome after cardiac arrest [414]. However, there is still uncertainty about what are the optimal timings and thresholds. Evidence from our review showed that, while prediction with $0 \%$ FPR can be achieved anytime from $24 \mathrm{~h}$ to 7 days after ROSC, the sensitivity of an individual NSE measurement for prediction of poor neurological outcome with $0 \%$ FPR is highest at 48-72 h after ROSC [15]. However, our review confirmed that the NSE threshold value for 0\% FPR is inconsistent because of a few patients with good neurological outcome despite very-high NSE values. The presence of these outliers can be partly explained with a release of NSE from extracerebral sources, such as red blood cells or neuroendocrine tumours. Repeated blood sampling and careful exclusion of extracerebral sources is recommended when using NSE for neuroprognostication. Another cause of variability for the NSE thresholds is represented by the different measurement techniques used [381]. In our 2020 review, the highest recorded NSE thresholds for 0\% FPR at 48 and $72 \mathrm{~h}$ from ROSC were $120 \mu \mathrm{g} \mathrm{L}^{-1}$ and $79 \mu \mathrm{g} \mathrm{L}{ }^{-1}$, respectively. However, these data refer to outliers, and in most studies the $0 \%$ FPR threshold was $60 \mu \mathrm{g} \mathrm{L}^{-1}$ and $50 \mu \mathrm{g} \mathrm{L}^{-1}$, respectively. Based on these data, we presume that the risk of a false positive prediction associated with an NSE value of $60 \mu \mathrm{g} \mathrm{L}^{-1}$ is minimal, especially because the NSE signal should be confirmed by at least another predictor. Nevertheless, we suggest that hospital laboratories using NSE create their own normal values and cut-off levels based on the test kit used. Increasing NSE values between 24 and $48 \mathrm{~h}$ or between $24 / 48 \mathrm{~h}$ and $72 \mathrm{~h}$ also suggests a poor outcome even if the incremental prognostic value of adding NSE trends to a single NSE value is uncertain [15, $375,379]$. We suggest performing serial NSE samples at 24, 48 and $72 \mathrm{~h}$ after ROSC so that NSE trends can be detected and confounding from occasional haemolysis can be minimised.

Signs of diffuse and extensive hypoxic-ischaemic brain injury on brain CT include an effacement of cortical sulci and reduced ventricle size (mainly from vasogenic oedema) and a reduced density of the grey matter with reduction or loss of the grey matter/white matter (GM/ WM) interface due to cytotoxic oedema. In the review informing these guidelines, the first sign was evaluated qualitatively in one study [394], based on visual inspection from a neuroradiologist, while most studies assessed the reduced GM/WM interface as the ratio of the densities of the grey matter and the white matter (GWR) measured in Hounsfield units. This was generally done within $2 \mathrm{~h}$ from ROSC, but some studies assessed GWR within $24 \mathrm{~h}[301,386]$, and one within $72 \mathrm{~h}$ [388]. As for other predictors based on continuous variables, the GWR thresholds for 0\% FPR varied across studies, presumably because of variations in the methods for GWR calculation, or in the software or scanner' characteristics [15].

Hypoxic-ischaemic brain injury reduces water diffusivity, which appears on magnetic resonance imaging (MRI) as a hyperintensity on diffusion weighted imaging 
(DWI) with corresponding low apparent diffusion coefficient (ADC) values. In severe hypoxic-ischaemic brain injury, hyperintensity on DWI involves the cerebral cortex extensively and the basal ganglia. Measurement of ADC enables a quantitative assessment of the severity of diffusion changes. In studies on prognostication after cardiac arrest, three methods for ADC measurement were described: the mean global or regional $A D C$ value of the brain [401], the proportion of voxels with low ADC [402] and the maximum size of the MRI clusters with minimum ADC [400]. All these studies identified ADC thresholds for $0 \%$ FPR, often with a corresponding high sensitivity. However, these thresholds were inconsistent across different areas of the brain within the same study and the same technique.

Because of the lack of standardisation in measurement methods and the lack of multicentre validation studies using comparable measurement techniques, we suggest that predictive indices based on neuroimaging are used only in places where specific experience is available. We also suggest that centres using neuroimaging for prognostication after cardiac arrest create their own normal values and threshold values based on the technique used.

When none of the criteria for poor outcome described above are present, neurological outcome remains indeterminate (Fig. 4). We, therefore, suggest observing and re-evaluating patients with indeterminate outcome over time in order to detect signs of awakening. In three studies conducted in resuscitated comatose patients treated with TTM for $24 \mathrm{~h}$, the prevalence of late awakening, defined as a recovery of consciousness at $\geq 48 \mathrm{~h}$ from suspension of sedation was 20/89 (22\%) [415], 56/194 (29\%) [305] and 78/228 (34\%) [204]. Last awakening occurred on day 11, day 12 and day 23 from suspension of sedation, respectively. In two other studies, the last patient awoke on day 22 and day 29 [403, 416]. Organ dysfunction, such as post-resuscitation shock or renal failure $[204,305]$ and use of midazolam instead of propofol for sedation $[204,265]$ were associated with a higher likelihood of late awakening, which suggests that at least some of these cases may have been due to a reduced clearance of sedation. In a before-and-after study comparing two sedative regimens (propofol-remifentanil versus midazolam-fentanyl) in 460 comatose resuscitated patients undergoing TTM, use of propofol-remifentanil was associated with significantly lower odds of delayed awakening after adjustment [OR $0.08(0.03-0.2)$ ] [305], confirming indirect evidence from a previous smaller study [264].

Late awakening does not preclude full neurological recovery. However, the likelihood of awakening in resuscitated patients who remain comatose decreases progressively with time and the rates of good neurological outcome are generally lower in late vs. early awakeners $[204,305,416]$.

The present guidelines apply only to neurological prognostication. Besides hypoxic-ischaemic brain injury, other, albeit less common, causes of death in resuscitated comatose patients include cardiovascular instability [23], and multiple organ failure [303, 304]. These factors may result in treatment limitations independently from the patient's neurological status or cause non-neurological death even after neurological recovery has occurred [295, $307,417]$. In clinical practice, a comprehensive prognostic approach in resuscitated comatose patients should inevitably consider the role of extracerebral factors as well as patient characteristics such as age, comorbidities, and functional status.

\section{Withdrawal of life-sustaining therapy}

While a minority of the resuscitated patients treated in an ICU die during the first few days due to cardiovascular collapse or massive brain swelling causing brain death, most deaths will be secondary to a decision to withdraw life-sustaining therapy (WLST) [22, 23, 26, 303]. Generally, a presumption that the final neurological outcome of the patient will be poor is central to this decision [26]. Pre-existing co-morbidities may also contribute to a WLST decision [22]. The clinical team discussing the prognosis of an individual patient need to consider that inaccurately pessimistic prognostication could lead to WLST in patients who might otherwise achieve a good functional outcome but also that overly conservative prognostication could leave patients in a severely disabled state undesired by themselves and their relatives [418]. Patients may not receive specific treatments because they are not available, or because there is an active decision to withhold them. The main reasons for withholding treatments are that they will not benefit the patient or, if known, the patient's wishes not to have a specific treatment $[418,419]$. There are few specific data on withholding life sustaining therapies in post-cardiac arrest patients specifically.

The practice of WLST varies widely across Europe and impacts the proportion of CA-patients surviving with severe brain injury (CPC 3-4). Lacking highquality data, this fraction appears to vary widely from approximately $10-50 \%$ [243, 300, 417]. The most apparent effects are seen for patients who remain in an unresponsive wakefulness/vegetative state (CPC 4). As an example, $1 / 243(0.4 \%)$ survivors in a northern European study [243] compared with $61 / 195(31 \%)$ in an Italian multi-centre study [300] were in CPC 4 at 6 months. Evidence for variation in WLST practice across Europe was also found in the Ethicus Study: physicians from southern Europe were less prone to withdraw treatment 
compared with those from northern Europe, and there was also an effect of religion [420]. The Ethicus-2 Study has shown that the frequency of WLST and withholding decisions among general ICU patients has increased over the past 15-20 years [421].

Recent studies, based on propensity score matching, indicate that premature $(<72 \mathrm{~h})$ WLST for neurological reasons are common and may be the cause of death for a substantial proportion of patients who might have recovered to a good outcome if their intensive care treatment had been prolonged [422, 423]. The brain stem is more resistant to hypoxic-ischaemic injury than the cerebrum and the recovery of functions such as spontaneous breathing and sleep-wake cycle is part of the trajectory towards an unresponsive wakefulness/vegetative syndrome. The period when the patient is still dependent on intensive care is sometimes referred to as the 'window of opportunity for death' [424]. This perception may cause a sense of urgency for the relatives and treating team indirectly impacting decisions on premature WLST $[425,426]$. One qualitative study identified limitations in family-team communication as an important factor for premature WLST after cardiac arrest [426]. Caregivers' inappropriate avoidance of uncertainty may also be important, leading to overly pessimistic perceptions of the prognosis [427].

Although some tests show high specificity for predicting a poor outcome before $72 \mathrm{~h}$, we recommend that, in general, conclusions about the neurological prognosis are postponed until at least $72 \mathrm{~h}$ after the cardiac arrest and the influence of sedative and metabolic factors have been ruled out. This will enable most patients with good outcome to awaken before the prognostic assessment, decreasing the risk of false predictions [265]. We encourage local protocols on how to collect information about the extent of brain injury during the first days. Use all available resources to inform a multimodal assessment $[9,15]$. Relatives will require regular clear and structured information and an understanding of their role in decision-making. Early indicators of poor prognosis may be conveyed in a balanced fashion to inform relatives that the situation is grave and enable time for adjustment before critical decisions are made. The bedside nurses are confronted by grieving caregivers, which may be very stressful [426]. Allocate sufficient time for communication around the prognosis within the team and with the relatives [428].

While the assessment of post-cardiac arrest neurological prognosis and discussions about WLST are most often linked, try to separate these processes in discussions and documentation. Decisions about WLST need to consider several aspects other than the perceived brain injury; for example, age, co-morbidities and the prognosis for general organ function [22]. Consequently, for ethical reasons, WLST may be considered for patients in whom the neurological prognosis is uncertain or even favourable. Conversely, intensive care may be prolonged despite dismal neurological prognosis because absolute certainty is unobtainable for an individual patient [429]. The patient's preferences are central. Since the patient cannot be asked and advance directives are rare among cardiac arrest victims, the relatives are usually the primary source of information about the patient's likely wishes.

\section{Long-term outcome after cardiac arrest Long-term outcome}

In countries where WLST is not practiced widely, poor outcome because of hypoxic-ischaemic brain injury is common $[387,430]$. The prognosis of patients who are still comatose or in an unresponsive wakefulness state 1 month after the cardiac arrest is poor and they rarely recover [430, 431]. In contrast, in countries practising WLST, the majority of survivors are defined as having a 'good' neurological outcome based on global outcome measures such as Cerebral Performance Categories (CPC), modified Rankin Scale (mRS) or the Glasgow Outcome Scale/Extended (GOS/E) [290, 412, 432-434]. However, these measures are not sufficiently sensitive to capture the problems that many of the survivors experience, including cognitive, emotional and physical problems and fatigue [435-437]. In fact, approximately $40-50 \%$ of the survivors have long-term cognitive impairments [229, 438, 439]. Impairments are mostly mild to moderate and, although all cognitive domains can be affected, most problems are seen in memory, attention, processing speed and executive functioning (e.g. planning, organisation, initiation, flexibility) [229, 435, 438440]. In general, most cognitive recovery occurs during the first 3 months after the cardiac arrest [441-443].

Emotional problems are also common. 3-6 months after the cardiac arrest anxiety is present in 15-30\% of the survivors and remains in $15-23 \%$ at 12 months [444-446]. Depressive symptoms range from 13 to $32 \%$ at 3-6 months and decrease to $5-15 \%$ at 12 months [ $444-$ 447]. Symptoms of post-traumatic stress remain in about a quarter of the survivors [436, 444, 447, 448]. Furthermore, some survivors show behavioural problems, such as aggressive/uninhibited behaviour or emotional lability [439].

Fatigue is also frequently reported and is present in approximately $70 \%$ of the survivors at 6 months and remains in half of the survivors 1 year after the event $[444,449,450]$. Physical problems, including rib fractures, muscle weakness and ambulation difficulties, have also been reported [437, 444, 451, 452]. However, 
the impact of survival on physical function has received little attention; when compared with age and gendermatched populations, reduced physical functioning has been reported in survivors at 3 months [453], 6 months [452], 12 months [434] and 3 years [451]. Almost half of survivors report limitations because of physical difficulties at 6 months [452], with up to $40 \%$ describing mobility problems $[434,439,444,454]$ and limitations in usual activities at 12 months [434, 444, 454].

After discharge, most survivors are able to return home and only a small percentage $(1-10 \%)$ need to be admitted to a long-term care facility [444, 454, 455]. The large majority $(82-91 \%)$ are independent in their basic activities of daily living (ADL) [228, 438, 451, 454]. Although most survivors are able to resume their pre-arrest activities, they experience more restrictions in societal participation compared with myocardial infarction patients [444, 450]. Cognitive impairments, depression, fatigue and restricted mobility are negative predictors for future participation [450].

Of those who were previously working, $63-85 \%$ are able to return to work, although some need to adapt their working hours or activities [434, 444, 450, 451, 454, 456-458]. Decreased likelihood of return to work is associated with cognitive problems and fatigue, unwitnessed OHCA, absence of bystander CPR, female gender, higher age and lower socio-economic status [450, 453, 456-458].

Cognitive impairments, emotional problems and female gender are associated with a lower quality of life [434, 442, 452, 453, 459-464]. However, general health-related quality of life is, on average, reported as good with overall scores approaching normal population values, as was shown in two systematic reviews and confirmed in several more recent studies [228, 434, $454,465,466]$. Such generic assessments lack sufficient granularity to comprehensively capture the breadth of problems experienced by survivors, with the result that the impact of cardiac arrest survival may be incompletely captured [290]. Supplementing such generic assessment with condition or problem-specific assessment is recommended [290].

More detailed information on recovery and long-term outcome after cardiac arrest, as well as a description of the current rehabilitation practices in Europe can be found in the epidemiology section of the 2021 European Resuscitation Council Guidelines [467].

\section{In-hospital assessment and follow-up after hospital discharge}

Early rehabilitation and assessment during hospital phase There are no studies of early rehabilitation interventions for cardiac arrest survivors specifically but there is sub- stantial overlap with the post-intensive care syndrome (PICS). For other ICU patients, interventions of early mobilisation and prevention of delirium are described, and similar interventions are thought to be useful for cardiac arrest patients as well $[437,468-470]$. Recommendations in the UK National Institute for Health and Care Excellence (NICE) guidelines for rehabilitation after critical illness suggest that individualised rehabilitation plans and information should be provided before discharge from the ICU and the hospital, based on functional assessments of physical and non-physical (e.g. cognitive and emotional) impairments [471]. However, a recent AHA Scientific Statement focusing on survivorship highlights that discharge planning and organisation of further rehabilitation needs after cardiac arrest is often lacking [437].

We, therefore, recommend providing information and performing functional assessments of physical and nonphysical impairments before discharge from the hospital to identify potential rehabilitation needs and arrange referral for rehabilitation if indicated (Fig. 5).

\section{Follow-up and screening after hospital discharge}

Although cognitive impairments, emotional problems and fatigue are common after cardiac arrest, these invisible problems' are not always recognised by healthcare professionals [442, 450, 453, 457, 464]. Since these problems have a significant impact on long-term outcome and quality of life, follow-up should be organised in such a way that these problems are detected early enabling appropriate care or rehabilitation to be arranged [472-474].

Evidence on this subject is scarce but results from one $\mathrm{RCT}$ showed that an early intervention service for cardiac arrest survivors and their caregivers improved emotional well-being and quality of life, resulted in a faster return to work and was cost-effective $[475,476]$. This individualised programme is provided by a specialised nurse, starts soon after discharge from the hospital and comprises one to six consultations during the first 3 months. The intervention consists of screening for cognitive and emotional problems, provision of information and support and referral to further specialised care if indicated [477, 478]. There are several other examples of how follow-up after cardiac arrest can be organised [474, 479, 480]. UK NICE guidelines for rehabilitation after critical illness likewise recommend a follow-up and reassessment for physical and non-physical problems 2-3 months after discharge to enable identification of remaining problems and to provide further support as needed [471]. For cardiac arrest survivors, reassessments have also been suggested at 3, 6 and 12 months [437].

We, therefore, suggest the systematic follow-up of all cardiac arrest survivors within 3 months following 
hospital discharge which should, at least, include cognitive screening, screening for emotional problems and fatigue and the provision of information and support for patients and their family (Fig. 5).

\section{Screening for cognitive problems}

To screen for cognition, the patient can be asked about common cognitive complaints, such as memory problems, attention difficulties, distractibility, slowness in thinking, irritability and problems in initiation, planning, multi-tasking or flexibility. Family members can also provide useful insight into changes in cognition and behaviour. A structured questionnaire, such as the Informant Questionnaire of Cognitive decline in the Elderly-Cardiac Arrest version (IQCODE-CA) or the Checklist Cognition and Emotion (CLCE-24), may be used [481, 482]. Formal cognitive screening is recommended because patients are not always aware of their cognitive impairments [443, 472, 483]. We suggest use of the Montreal Cognitive Assessment (MoCA) tool, which takes approximately $10 \mathrm{~min}$ to administer, is easy to use and available in many languages (see www.mocatest.org) [480, 483-485]. If there are signs of cognitive impairment, consider referral to a neuropsychologist for more extensive neuropsychological assessment or another specialist in cognitive rehabilitation, such as an occupational therapist, should be considered [486].

\section{Screening for emotional problems and fatigue}

To screen for emotional problems, the presence of emotional symptoms, including symptoms of anxiety, depression and posttraumatic stress, can be explored. Questionnaires, such as the Hospital Anxiety and Depression Scale (HADS), may be useful [437, 473, 480, 487]. If severe emotional problems are detected we suggest referral to a psychologist or psychiatrist for further evaluation and treatment. We also suggest assessing the presence of fatigue; however, assessment guidance in this population is currently lacking. In case of severe fatigue consider referral to a specialist in rehabilitation medicine for advice on appropriate care.

Provision of information and support for survivor and family members

Exploring the need for and subsequent provision of appropriate information to patients and their family, preferably both in oral and written form, is recommended [488]. The active engagement of survivors and their family members to better understand their needs and how they would like to receive such information, is recommended as part of this process [437]. Information should cover not only medical subjects such as cardiac disease, risk factors, medication and ICD, but can also address other topics such as potential physical, cognitive and emotional changes and fatigue, resuming daily activities, driving and work, relationship and sexuality [477, 488-491].

It is also important to monitor the well-being of family members because the impact and burden can be substantial [490, 492]. Partners often have emotional problems, including symptoms of anxiety and posttraumatic stress, especially in women and those who witnessed the resuscitation [493, 494]. Consider referral to a social worker, psychologist or psychiatrist when indicated.

\section{Rehabilitation after cardiac arrest In-patient neurological rehabilitation}

In the presence of significant hypoxic-ischaemic brain injury, patients may require inpatient neurological rehabilitation and, although the evidence is limited, several small retrospective studies have shown that functional improvements can be achieved, reducing the burden of care on the family and society [495-497].

Although specific guidelines and evidence for neurological rehabilitation after cardiac arrest is lacking, there is more evidence and multiple clinical practice guidelines for other types of acquired brain injury such as traumatic brain injury and stroke which can guide the treatment of patients with hypoxic-ischaemic brain injury due to cardiac arrest [498-500]. These guidelines provide practical recommendations on topics such as motor function, physical rehabilitation, cognition, communication, activities of daily living and psychosocial issues. Guidelines on rehabilitation after critical illness/post-intensive care syndrome (PICS) can also be useful [471, 501-503].

\section{Cardiac rehabilitation}

Many cardiac arrest survivors are eligible to enrol in a cardiac rehabilitation programme [504]. There is evidence that cardiac rehabilitation reduces cardiovascular mortality and hospital admissions, improves quality of life, and is cost-effective [504-507]. Cardiac rehabilitation programmes are mostly generic programmes, in which patients with different cardiac diseases, e.g. postacute coronary syndrome, heart failure or post cardiac surgery, can participate. It involves exercise training, risk factor management, lifestyle advice, education and psychological support [505]. Cardiac rehabilitation is frequently offered as a centre-based out-patient service, but can also be organised in a home-based setting in combination with telemonitoring [508]. In specific cases it can be provided as an inpatient programme [505]. Not all cardiac arrest survivors are eligible for or have access to cardiac rehabilitation, either because of the cause of the cardiac arrest or because of variation in national or insurance policies [509]. 
Within cardiac rehabilitation programmes little attention is paid to potential cognitive problems. Among cardiac patients in general, cognitive and emotional problems have not been addressed well in cardiac rehabilitation programmes [510-512]. For cardiac arrest survivors, there are some examples in which cardiac and cognitive rehabilitation have been integrated, although evidence of effects is still lacking $[474,480]$.

\section{Cognitive rehabilitation, fatigue management and psychosocial interventions}

The goal of cognitive rehabilitation is to reduce the impact of cognitive impairments and to improve overall well-being and daily functioning [513]. It can include additional neuropsychological assessment to get more insight into the nature and severity of the cognitive impairments and other influencing factors. Extensive patient education is essential to give the patient and their family more insight into what has changed in their cognition and behaviour. Compensation strategies, such as memory strategy training and metacognitive strategy training (e.g. self-monitoring, self-regulation and planning ahead) and the use of external (memory) aids may be helpful [486]. Although there are no specific studies on the effects of cognitive rehabilitation in patients with brain injury caused by cardiac arrest, a recent evidencebased review on cognitive rehabilitation after stroke and traumatic brain injury, can serve as a guideline [486].

Fatigue management can be included in cognitive rehabilitation or provided alone [514, 515]. There is weak evidence that a 4-week telephone intervention, based on energy conservation and problem-solving therapy, can be of benefit for cardiac arrest survivors with moderate to severe fatigue [516, 517].

There is also evidence that psychosocial interventions specifically designed for cardiac arrest survivors can be valuable. Two RCTs showed benefit from nurse-led psychosocial interventions, either by telephone or faceto-face [518, 519]. These interventions addressed selfmanagement, coping strategies, relaxation, information and health education $[519,520]$.

There are currently no studies on the effectiveness of social support networks or virtual/online forums, but these may have additional value as a new and easily accessible form of psychosocial support and information after cardiac arrest [437].

\section{Organ donation}

Comatose post cardiac arrest patients who do not survive have the potential to become organ donors. This is important as demand for organs exceeds supply [521]. Post cardiac arrest patients are an increasing source of solid organ donors [522]. This guideline supports giving the opportunity for organ donation to patients and families when brain death occurs or there is a decision to withdraw life sustaining treatment.

This guideline specifically addresses the organ donation pathways following neurological (brain) death or controlled donation after circulatory death (Maastricht category III donors) in patients that achieve ROSC or are treated with E-CPR [523] (Fig. 6). Uncontrolled donation after circulatory death uDCD (Maastricht category I/II donors) is addressed in the Advanced Life Support section of the guidelines [523].

A previous 2015 ILCOR CoSTR and an ILCOR Scientific Statement on organ donation following CPR underpin this guideline [122]. Recent CPR should not prevent organ donation. Observational studies show that organs (heart, lung, kidney, liver, pancreas, intestine) from donors who have had CPR have similar graft survival rates compared with donors who have not had CPR [524, 525].

A systematic review identified 26 studies that showed the prevalence of brain death in comatose ventilated patients with hypoxic-ischaemic brain injury who died following CPR was $12.6 \%$ (95\% CI 10.2-15.2\%) with a higher prevalence following eCPR [27.9\% (19.7-36.6\%) vs. $8.3 \%(6.5-10.4 \%)]$ and that approximately $40 \%$ of these proceeded to organ donation [286]. The median time to diagnose brain death was 3.2 days. This systematic review concluded that patients who are unconscious after resuscitation from cardiac arrest, especially when resuscitated using e-CPR, should be assessed for signs of brain death.

Furthermore, in those who do not fulfil criteria for neurological death, WLST because of a poor neurological prognosis is a common cause of death. After OHCA, approximately two-thirds of deaths will be following WLST because of a poor neurological prognosis [22, 23]. This group of patients provides an increasing source of donors following controlled donation after circulatory death [526].

There is variation between countries regarding organ donation practices and clinicians must follow local legal and ethical requirements.

\section{Investigating sudden unexplained cardiac arrest}

Many sudden cardiac death victims have silent structural heart disease, most often coronary artery disease, but also primary arrhythmia syndromes, cardiomyopathies, familial hypercholesterolaemia and premature ischaemic heart disease. In the course of an autopsy of victims of sudden unexplained death (SUD), blood or tissue samples should be taken and stored for future genetic analysis [527]. Screening for genetic disorders is crucial for primary prevention in relatives as it may enable 
preventive antiarrhythmic treatment and medical followup [528-530]. A multidisciplinary cardiogenetic team should perform the family investigation. Initial evaluation may include clinical examination, electrophysiology and cardiac imaging. A genetic test should be considered according to the combination the results of cardiac family screening and pathology findings. The genetic test should be performed initially on the DNA of the deceased and testing of relatives should then be offered if a pathogenic or likely pathogenic variant is identified $[527,531]$. Given the implications for relatives, there may be local ethical guidelines for genetic testing.

\section{Cardiac arrest centres}

There is wide variation among hospitals in the availability and type of post resuscitation care, as well as clinical outcomes [532-534]. Cardiac arrest centres are hospitals providing evidence-based resuscitation treatments including emergency interventional cardiology, and bundled critical care with targeted temperature management, and protocolised cardiorespiratory support and prognostication [122, 207]. An expert consensus paper published by the Association of Acute Cardiovascular Care (ACVA), and many other European organisations including the ERC and ESICM, states that the minimum requirements for a cardiac arrest centre are 24/7 availability of an on-site coronary angiography laboratory, an emergency department, an intensive care unit (ICU), imaging facilities, such as echocardiography, computed tomography and magnetic resonance imaging [16].

ILCOR suggests that wherever possible, adult patients with non-traumatic OHCA cardiac arrest should be cared for in cardiac arrest centres [17]. This weak recommendation is based on very-low-certainty evidence from a systematic review that included 21 observational studies [535-555] and 1 pilot randomised trial [556]. Seventeen of these studies were included in a meta-analysis that found that patients cared for at cardiac arrest centres had improved survival to hospital discharge with favourable neurological outcome, but this was non-significant at 30 days [535-541, 545-552, 554, 555].

One observational study reported higher adjusted patient survival associated with direct transfer to a cardiac arrest centre compared with secondary interfacility transfer [552], but two other studies making the same comparisons report no difference in adjusted survival [536, 541]. One observational study reported higher adjusted survival in patients who underwent secondary transfer to a cardiac arrest centre compared with remaining at the initial non-cardiac arrest centre [550].
Supplementary Information

The online version contains supplementary material available at https://doi. org/10.1007/s00134-021-06368-4.

\begin{abstract}
Author details
${ }^{1}$ University of Warwick, Warwick Medical School, Coventry CV4 7AL, UK. ${ }^{2}$ Royal United Hospital, Bath BA1 3NG, UK. ${ }^{3}$ Department of Intensive Care, Emergency Medicine and Anaesthesiology, Fondazione Policlinico Universitario A. Gemelli-IRCCS, Rome, Italy. ${ }^{4}$ Institute of Anaesthesiology and Intensive Care Medicine, Università Cattolica del Sacro Cuore, Rome, Italy. ${ }^{5}$ Department of Anaesthesiology and Intensive Care Medicine, University Hospital of Cologne, Kerpener Straße 62, 50937 Cologne, Germany. ${ }^{6}$ Cochin University Hospital (APHP) and University of Paris (Medical School), Paris, France.

${ }^{7}$ Department of Clinical Sciences, Neurology, Lund University, Skane University Hospital, Lund, Sweden. ${ }^{8}$ Department of Clinical Sciences, Anaesthesia and Intensive Care Medicine, Lund University, Skane University Hospital, Lund, Sweden. ${ }^{9}$ Acute Medicine Research Pole, Institute of Experimental and Clinical Research (IREC), Université Catholique de Louvain, Brussels, Belgium.

${ }^{10}$ Emergency Department, University Hospitals Saint-Luc, Brussels, Belgium.

${ }^{11}$ Warwick Research in Nursing, Division of Health Sciences, Warwick Medical School, University of Warwick, Room A108, Coventry CV4 7AL, UK. ${ }^{12}$ Department of Clinical Sciences Lund, Neurology, Lund University, Skane University Hospital, Lund, Sweden. ${ }^{13}$ Department of Rehabilitation Medicine, University Medical Center Groningen, University of Groningen, Groningen, The Netherlands. ${ }^{14}$ Cardiology Department, Konstantopouleio General Hospital, Athens, Greece. ${ }^{15}$ Department of Anesthesiology, Oslo University Hospital and Institute of Clinical Medicine, University of Oslo, Oslo, Norway. ${ }^{16}$ Department of Emergency Care and Services, University of Helsinki and Helsinki University Hospital, Helsinki, Finland. ${ }^{17}$ Department of Intensive Care, Hôpital Erasme, Université Libre de Bruxelles, Route de Lennik, 808, 1070 Brussels, Belgium. 18 Southmead Hospital, North Bristol NHS Trust, Bristol BS10 5NB, UK.
\end{abstract}

\section{Declarations}

\section{Conflicts of interest}

JPN: Editor in Chief Resuscitation. CS: Associate Editor, Intensive Care Medicine. BWB: Treasurer of the European Resuscitation Council (ERC); Chairman of the German Resuscitation Council (GRC); Member of the Advanced Life Support (ALS) Task Force of the International Liaison Committee on Resuscitation (ILCOR); Member of the Executive Committee of the German Interdisciplinary Association for Intensive Care and Emergency Medicine (DIVI), Founder of the Deutsche Stiftung Wiederbelebung; Associate Editor of the European Journal of Anaesthesiology (EJA), Co-Editor of Resuscitation; Editor of Notfall + Rettungsmedizin, Co-Editor of the Brazilian Journal of Anesthesiology. Received fees for lectures from the following companies: Forum für medizinische Fortbildung (FomF), Baxalta Deutschland GmbH, ZOLL Medical Deutschland GmbH, C.R. Bard GmbH, GS Elektromedizinische Geräte G. Stemple GmbH, Novartis Pharma GmbH, Philips GmbH Market DACH, Bioscience Valuation BSV $\mathrm{GmbH}$. AC: Speaker's Fee from Bard Medical. TC: no conflicts of interest. HF: no conflicts of interest. CG: no conflicts of interest. GL: no conflicts of interest. VRMM: no conflicts of interest. NN: no conflicts of interest. TMO: no conflicts of interest. MBS: Speaker's Fee from Bard Medical (Ireland). FST: Speaker's Fees from BD and Zoll. JS: Editor, Resuscitation.

\section{Publisher's Note}

Springer Nature remains neutral with regard to jurisdictional claims in published maps and institutional affiliations.

Received: 30 November 2020 Accepted: 8 February 2021 Published online: 25 March 2021

\section{References}

1. Nolan JP, Soar J, Cariou A et al (2015) European Resuscitation Council and European Society of Intensive Care Medicine Guidelines for post-resuscitation Care 2015: section 5 of the European Resuscitation Council Guidelines for Resuscitation 2015. Resuscitation 95:202-222 
2. Nolan JP, Soar J, Cariou A et al (2015) European Resuscitation Council and European Society of Intensive Care Medicine 2015 guidelines for post-resuscitation care. Intensive Care Med 41:2039-2056

3. Nolan JP, Hazinski MF, Aickin R et al (2015) Part 1: executive summary: 2015 international consensus on cardiopulmonary resuscitation and emergency cardiovascular care science with treatment recommendations. Resuscitation 95:e1-e31

4. Olasveengen TM, de Caen AR, Mancini ME et al (2017) 2017 International consensus on cardiopulmonary resuscitation and emergency cardiovascular care science with treatment recommendations summary. Resuscitation 121:201-214

5. Soar J, Donnino MW, Maconochie I et al (2018) 2018 international consensus on cardiopulmonary resuscitation and emergency cardiovascular care science with treatment recommendations summary. Resuscitation 133:194-206

6. Soar J, Maconochie I, Wyckoff MH et al (2019) 2019 international consensus on cardiopulmonary resuscitation and emergency cardiovascular care science with treatment recommendations. Resuscitation 145:95-150

7. Nolan JP, Maconochie I, Soar J et al (2020) Executive summary 2020 international consensus on cardiopulmonary resuscitation and emergency cardiovascular care science with treatment recommendations. Resuscitation 156:A1-A22

8. Morley PT, Atkins DL, Finn JC et al (2020) Evidence evaluation process and management of potential conflicts of interest. Resuscitation 156:A23-A34

9. Soar J, Berg KM, Andersen LW et al (2020) Adult advanced life support: 2020 international consensus on cardiopulmonary resuscitation and emergency cardiovascular care science with treatment recommendations. Resuscitation 156:A80-A119

10. Lemkes JS, Janssens GN, van der Hoeven NW et al (2019) Coronary angiography after cardiac arrest without ST-segment elevation. N Engl J Med 380:1397-1407

11. Collet JP, Thiele H, Barbato E, et al. (2020) 2020 ESC Guidelines for the management of acute coronary syndromes in patients presenting without persistent ST-segment elevation. Eur Heart J. https://doi.org/10. 1093/eurheartj/ehaa575

12. Kapur J, Elm J, Chamberlain JM et al (2019) Randomized trial of three anticonvulsant medications for status epilepticus. N Engl J Med 381:2103-2113

13. Lascarrou JB, Merdji H, Le Gouge A et al (2019) Targeted temperature management for cardiac arrest with nonshockable rhythm. N Engl J Med 381:2327-2337

14. Dankiewicz J, Cronberg T, Lilja G et al (2019) Targeted hypothermia versus targeted Normothermia after out-of-hospital cardiac arrest (TTM2): a randomized clinical trial-Rationale and design. Am Heart J 217:23-31

15. Sandroni C, D'Arrigo S, Cacciola S et al (2020) Prediction of poor neurological outcome in comatose survivors of cardiac arrest: a systematic review. Intensive Care Med 46:1803-1851

16. Sinning C, Ahrens I, Cariou A et al (2020) The cardiac arrest centre for the treatment of sudden cardiac arrest due to presumed cardiac cause aims, function and structure: Position paper of the Association for Acute CardioVascular Care of the European Society of Cardiology (AVCV), European Association of Percutaneous Coronary Interventions (EAPCI), European Heart Rhythm Association (EHRA), European Resuscitation Council (ERC), European Society for Emergency Medicine (EUSEM) and European Society of Intensive Care Medicine (ESICM). Eur Heart J Acute Cardiovasc Care 9:S193-S202

17. Yeung J, Matsuyama T, Bray J, Reynolds J, Skrifvars MB (2019) Does care at a cardiac arrest centre improve outcome after out-of-hospital cardiac arrest? A systematic review. Resuscitation 137:102-115

18. Nolan JP, Neumar RW, Adrie C et al (2008) Post-cardiac arrest syndrome: epidemiology, pathophysiology, treatment, and prognostication. A Scientific Statement from the International Liaison Committee on Resuscitation; the American Heart Association Emergency Cardiovascular Care Committee; the Council on Cardiovascular Surgery and Anesthesia; the Council on Cardiopulmonary, Perioperative, and Critical Care; the Council on Clinical Cardiology; the Council on Stroke. Resuscitation 79:350-379
19. Mongardon N, Dumas F, Ricome S et al (2011) Postcardiac arrest syndrome: from immediate resuscitation to long-term outcome. Ann Intensive Care 1:45

20. Stub D, Bernard S, Duffy SJ, Kaye DM (2011) Post cardiac arrest syndrome: a review of therapeutic strategies. Circulation 123:1428-1435

21. Sekhon MS, Ainslie PN, Griesdale DE (2017) Clinical pathophysiology of hypoxic ischemic brain injury after cardiac arrest: a "two-hit" model. Crit Care 21:90

22. Witten L, Gardner R, Holmberg MJ et al (2019) Reasons for death in patients successfully resuscitated from out-of-hospital and in-hospital cardiac arrest. Resuscitation 136:93-99

23. Lemiale V, Dumas F, Mongardon $\mathrm{N}$ et al (2013) Intensive care unit mortality after cardiac arrest: the relative contribution of shock and brain injury in a large cohort. Intensive Care Med 39:1972-1980

24. Laver S, Farrow C, Turner D, Nolan J (2004) Mode of death after admission to an intensive care unit following cardiac arrest. Intensive Care Med 30:2126-2128

25. Olasveengen TM, Sunde K, Brunborg C, Thowsen J, Steen PA, Wik L (2009) Intravenous drug administration during out-of-hospital cardiac arrest: a randomized trial. JAMA 302:2222-2229

26. Dragancea I, Rundgren M, Englund E, Friberg H, Cronberg T (2013) The influence of induced hypothermia and delayed prognostication on the mode of death after cardiac arrest. Resuscitation 84:337-342

27. Nielsen N, Wetterslev J, Cronberg T et al (2013) Targeted temperature management at 33 degrees $\mathrm{C}$ versus $36^{\circ} \mathrm{C}$ after cardiac arrest. $\mathrm{N}$ Engl J Med 369:2197-2206

28. Cha KC, Kim HI, Kim OH et al (2018) Echocardiographic patterns of postresuscitation myocardial dysfunction. Resuscitation 124:90-95

29. Jentzer JC, Anavekar NS, Mankad SV et al (2018) Changes in left ventricular systolic and diastolic function on serial echocardiography after out-of-hospital cardiac arrest. Resuscitation 126:1-6

30. Laurent I, Monchi M, Chiche JD et al (2002) Reversible myocardial dysfunction in survivors of out-of-hospital cardiac arrest. J Am Coll Cardiol 40:2110-2116

31. Ruiz-Bailen M, Aguayo de Hoyos E, Ruiz-Navarro S et al (2005) Reversible myocardial dysfunction after cardiopulmonary resuscitation. Resuscitation 66:175-181

32. Chalkias A, Xanthos T (2012) Pathophysiology and pathogenesis of post-resuscitation myocardial stunning. Heart Fail Rev 17:117-128

33. Wardi G, Blanchard D, Dittrich T, Kaushal K, Sell R (2016) Right ventricle dysfunction and echocardiographic parameters in the first $24 \mathrm{~h}$ following resuscitation in the post-cardiac arrest patient: a retrospective cohort study. Resuscitation 103:71-74

34. Cerchiari EL, Safar P, Klein E, Diven W (1993) Visceral, hematologic and bacteriologic changes and neurologic outcome after cardiac arrest in dogs. The visceral post-resuscitation syndrome. Resuscitation 25:119-136

35. Adrie C, Monchi M, Laurent I et al (2005) Coagulopathy after successful cardiopulmonary resuscitation following cardiac arrest: implication of the protein C anticoagulant pathway. J Am Coll Cardiol 46:21-28

36. Grimaldi D, Guivarch E, Neveux N et al (2013) Markers of intestinal injury are associated with endotoxemia in successfully resuscitated patients. Resuscitation 84:60-65

37. Roberts BW, Kilgannon JH, Chansky ME et al (2013) Multiple organ dysfunction after return of spontaneous circulation in postcardiac arrest syndrome. Crit Care Med 41:1492-1501

38. Bottiger BW, Bohrer H, Boker T, Motsch J, Aulmann M, Martin E (1996) Platelet factor 4 release in patients undergoing cardiopulmonary resuscitation - can reperfusion be impaired by platelet activation? Acta Anaesthesiol Scand 40:631-635

39. Bottiger BW, Motsch J, Braun V, Martin E, Kirschfink M (2002) Marked activation of complement and leukocytes and an increase in the concentrations of soluble endothelial adhesion molecules during cardiopulmonary resuscitation and early reperfusion after cardiac arrest in humans. Crit Care Med 30:2473-2480

40. Bottiger BW, Motsch J, Bohrer $\mathrm{H}$ et al (1995) Activation of blood coagulation after cardiac arrest is not balanced adequately by activation of endogenous fibrinolysis. Circulation 92:2572-2578

41. Viersen VA, Greuters S, Korfage AR et al (2012) Hyperfibrinolysis in out of hospital cardiac arrest is associated with markers of hypoperfusion. Resuscitation 83:1451-1455 
42. Duvekot A, Viersen VA, Dekker SE et al (2015) Low cerebral oxygenation levels during resuscitation in out-of-hospital cardiac arrest are associated with hyperfibrinolysis. Anesthesiology 123:820-829

43. Buchtele N, Schorgenhofer C, Spiel AO, Jilma B, Schwameis M (2018) Increased fibrinolysis as a specific marker of poor outcome after cardiac arrest. Crit Care Med 46:e995-e1001

44. Adrie C, Adib-Conquy M, Laurent I et al (2002) Successful cardiopulmonary resuscitation after cardiac arrest as a "sepsis-like" syndrome. Circulation 106:562-568

45. Adrie C, Laurent I, Monchi M, Cariou A, Dhainaou JF, Spaulding C (2004) Postresuscitation disease after cardiac arrest: a sepsis-like syndrome? Curr Opin Crit Care 10:208-212

46. Huet O, Dupic L, Batteux F et al (2011) Postresuscitation syndrome: potential role of hydroxyl radical-induced endothelial cell damage. Crit Care Med 39:1712-1720

47. Fink K, Schwarz M, Feldbrugge L et al (2010) Severe endothelial injury and subsequent repair in patients after successful cardiopulmonary resuscitation. Crit Care 14:R104

48. van Genderen ME, Lima A, Akkerhuis M, Bakker J, van Bommel J (2012) Persistent peripheral and microcirculatory perfusion alterations after out-of-hospital cardiac arrest are associated with poor survival. Crit Care Med 40:2287-2294

49. Bro-Jeppesen J, Kjaergaard J, Wanscher M et al (2015) Systemic inflammatory response and potential prognostic implications after out-of-hospital cardiac arrest: a substudy of the target temperature management trial. Crit Care Med 43:1223-1232

50. Sutherasan Y, Penuelas O, Muriel A et al (2015) Management and outcome of mechanically ventilated patients after cardiac arrest. Crit Care 19:215

51. Peberdy MA, Andersen LW, Abbate A et al (2016) Inflammatory markers following resuscitation from out-of-hospital cardiac arrest-a prospective multicenter observational study. Resuscitation 103:117-124

52. Bro-Jeppesen J, Johansson PI, Hassager C et al (2016) Endothelial activation/injury and associations with severity of post-cardiac arrest syndrome and mortality after out-of-hospital cardiac arrest. Resuscitation 107:71-79

53. Bro-Jeppesen J, Johansson PI, Kjaergaard J et al (2017) Level of systemic inflammation and endothelial injury is associated with cardiovascular dysfunction and vasopressor support in post-cardiac arrest patients. Resuscitation 121:179-186

54. Chelly J, Mongardon N, Dumas F et al (2012) Benefit of an early and systematic imaging procedure after cardiac arrest: insights from the PROCAT (Parisian Region Out of Hospital Cardiac Arrest) registry. Resuscitation 83:1444-1450

55. Arnaout M, Mongardon N, Deye N et al (2015) Out-of-hospital cardiac arrest from brain cause: epidemiology, clinical features, and outcome in a multicenter cohort. Crit Care Med 43:453-460

56. Inamasu J, Miyatake S, Tomioka H et al (2009) Subarachnoid haemorrhage as a cause of out-of-hospital cardiac arrest: a prospective computed tomography study. Resuscitation 80:977-980

57. Shin J, Kim K, Lim YS et al (2016) Incidence and clinical features of intracranial hemorrhage causing out-of-hospital cardiac arrest: a multicenter retrospective study. Am J Emerg Med 34:2326-2330

58. Legriel S, Bougouin W, Chocron R et al (2018) Early in-hospital management of cardiac arrest from neurological cause: diagnostic pitfalls and treatment issues. Resuscitation 132:147-155

59. Caputo ND, Stahmer C, Lim G, Shah K (2014) Whole-body computed tomographic scanning leads to better survival as opposed to selective scanning in trauma patients: a systematic review and meta-analysis. J Trauma Acute Care Surg 77:534-539

60. Lott CT, Alfonzo A, Barelli A, González-Salvado V, Hinkelbein J, Nolan JP Paal P, Perkins GD, Thies K-C, Yeung J, Zideman DA, Soar J. (2021) European Resuscitation Council Guidelines 2021: Cardiac arrest in specia circumstances. Resuscitation

61. Berg KM, Grossestreuer AV, Uber A, Patel PV, Donnino MW (2017) Intubation is not a marker for coma after in-hospital cardiac arrest: a retrospective study. Resuscitation 119:18-20

62. Benger JR, Kirby K, Black S et al (2018) Effect of a strategy of a supraglot tic airway device vs tracheal intubation during out-of-hospital cardiac arrest on functional outcome: the AIRWAYS-2 randomized clinical trial. JAMA 320:779-791
63. Higgs A, McGrath BA, Goddard C et al (2018) Guidelines for the management of tracheal intubation in critically ill adults. Br J Anaesth 120:323-352

64. Nolan JP, Kelly FE (2011) Airway challenges in critical care. Anaesthesia 66(Suppl 2):81-92

65. Miller M, Groombridge CJ, Lyon R (2017) Haemodynamic changes to a midazolam-fentanyl-rocuronium protocol for pre-hospital anaesthesia following return of spontaneous circulation after cardiac arrest. Anaesthesia 72:585-591

66. Holmberg MJ, Nicholson T, Nolan JP et al (2020) Oxygenation and ventilation targets after cardiac arrest: a systematic review and meta-analysis. Resuscitation 152:107-115

67. Fugate JE (2017) Anoxic-ischemic brain injury. Neurol Clin 35:601-611

68. Endisch C, Westhall E, Kenda M et al (2020) Hypoxic-ischemic encephalopathy evaluated by brain autopsy and neuroprognostication after cardiac arrest. JAMA Neurol 77(11):1-10

69. Llitjos JF, Mira JP, Duranteau J, Cariou A (2016) Hyperoxia toxicity after cardiac arrest: what is the evidence? Ann Intensive Care 6:23

70. Bougle A, Daviaud F, Bougouin W et al (2016) Determinants and significance of cerebral oximetry after cardiac arrest: a prospective cohort study. Resuscitation 99:1-6

71. Rosenthal G, Hemphill JC 3rd, Sorani M et al (2008) Brain tissue oxygen tension is more indicative of oxygen diffusion than oxygen delivery and metabolism in patients with traumatic brain injury. Crit Care Med 36:1917-1924

72. Liu Y, Rosenthal RE, Haywood Y, Miljkovic-Lolic M, Vanderhoek JY, Fiskum G (1998) Normoxic ventilation after cardiac arrest reduces oxidation of brain lipids and improves neurological outcome. Stroke 29:1679-1686

73. Pilcher J, Weatherall M, Shirtcliffe P, Bellomo R, Young P, Beasley R (2012) The effect of hyperoxia following cardiac arrest-a systematic review and meta-analysis of animal trials. Resuscitation 83:417-422

74. Investigators I-R, the A, New Zealand Intensive Care Society Clinical Trials G et al (2020) Conservative Oxygen Therapy during Mechanical Ventilation in the ICU. N Engl J Med 382:989-998

75. Jakkula P, Reinikainen M, Hastbacka J et al (2018) Targeting two different levels of both arterial carbon dioxide and arterial oxygen after cardiac arrest and resuscitation: a randomised pilot trial. Intensive Care Med 44:2112-212

76. Bray JE, Hein C, Smith K et al (2018) Oxygen titration after resuscitation from out-of-hospital cardiac arrest: a multi-centre, randomised controlled pilot study (the EXACT pilot trial). Resuscitation 128:211-215

77. Thomas M, Voss S, Benger J, Kirby K, Nolan JP (2019) Cluster randomised comparison of the effectiveness of 100\% oxygen versus titrated oxygen in patients with a sustained return of spontaneous circulation following out of hospital cardiac arrest: a feasibility study. PROXY: post ROSC OXYgenation study. BMC Emerg Med 19:16

78. Young P, Bailey M, Bellomo R et al (2014) HyperOxic Therapy OR NormOxic Therapy after out-of-hospital cardiac arrest (HOT OR NOT): a randomised controlled feasibility trial. Resuscitation 85:1686-1691

79. Kuisma M, Boyd J, Voipio V, Alaspaa A, Roine RO, Rosenberg P (2006) Comparison of 30 and the $100 \%$ inspired oxygen concentrations during early post-resuscitation period: a randomised controlled pilot study. Resuscitation 69:199-206

80. Young P, Mackle D, Bellomo R et al (2020) Conservative oxygen therapy for mechanically ventilated adults with suspected hypoxic ischaemic encephalopathy. Intensive Care Med 46:2411-2422

81. Humaloja J, Litonius E, Efendijev I et al (2019) Early hyperoxemia is not associated with cardiac arrest outcome. Resuscitation 140:185-193

82. Young P, Pilcher J, Patel M et al (2013) Delivery of titrated oxygen via a self-inflating resuscitation bag. Resuscitation 84:391-394

83. Nelskyla A, Parr MJ, Skrifvars MB (2013) Prevalence and factors correlating with hyperoxia exposure following cardiac arrest-an observational single centre study. Scand J Trauma Resusc Emerg Med 21:35

84. Storm C, Leithner C, Krannich A et al (2014) Regional cerebral oxygen saturation after cardiac arrest in 60 patients - a prospective outcome study. Resuscitation 85:1037-1041

85. Jakkula P, Hastbacka J, Reinikainen M et al (2019) Near-infrared spectroscopy after out-of-hospital cardiac arrest. Crit Care 23:171

86. Spindelboeck W, Gemes G, Strasser C et al (2016) Arterial blood gases during and their dynamic changes after cardiopulmonary resuscitation: a prospective clinical study. Resuscitation 106:24-29 
87. Mekontso Dessap A, Charron C, Devaquet J et al (2009) Impact of acute hypercapnia and augmented positive end-expiratory pressure on right ventricle function in severe acute respiratory distress syndrome. Intensive Care Med 35:1850-1858

88. Curley G, Kavanagh BP, Laffey JG (2010) Hypocapnia and the injured brain: more harm than benefit. Crit Care Med 38:1348-1359

89. Pynnonen L, Falkenbach P, Kamarainen A, Lonnrot K, Yli-Hankala A, Tenhunen J (2011) Therapeutic hypothermia after cardiac arrest—cerebral perfusion and metabolism during upper and lower threshold normocapnia. Resuscitation 82:1174-1179

90. Thompson BT, Chambers RC, Liu KD (2017) Acute respiratory distress syndrome. N Engl J Med 377:562-572

91. Serpa Neto A, Cardoso SO, Manetta JA et al (2012) Association between use of lung-protective ventilation with lower tidal volumes and clinical outcomes among patients without acute respiratory distress syndrome: a meta-analysis. JAMA 308:1651-1659

92. Johnson NJ, Caldwell E, Carlbom DJ et al (2019) The acute respiratory distress syndrome after out-of-hospital cardiac arrest: Incidence, risk factors, and outcomes. Resuscitation 135:37-44

93. Czerwinska-Jelonkiewicz K, Grand J, Tavazzi G, et al. (2020) Acute respiratory failure and inflammatory response after out-of-hospital cardiac arrest: results of the Post-Cardiac Arrest Syndrome (PCAS) pilot study. Eur Heart J Acute Cardiovasc Care 2048872619895126

94. Kim JS, Kim YJ, Kim M et al (2020) Impact of lung compliance on neurological outcome in patients with acute respiratory distress syndrome following out-of-hospital cardiac arrest. J Clin Med 9:527

95. Gonzalvo R, Marti-Sistac O, Blanch L, Lopez-Aguilar J (2007) Bench-tobedside review: brain-lung interaction in the critically ill-a pending issue revisited. Crit Care 11:216

96. Eastwood GM, Schneider AG, Suzuki S et al (2016) Targeted therapeutic mild hypercapnia after cardiac arrest: a phase II multi-centre randomised controlled trial (the CCC trial). Resuscitation 104:83-90

97. Vaahersalo J, Bendel S, Reinikainen M et al (2014) Arterial blood gas tensions after resuscitation from out-of-hospital cardiac arrest: associations with long-term neurologic outcome. Crit Care Med 42:1463-1470

98. Hope Kilgannon J, Hunter BR, Puskarich MA et al (2019) Partial pressure of arterial carbon dioxide after resuscitation from cardiac arrest and neurological outcome: a prospective multi-center protocol-directed cohort study. Resuscitation 135:212-220

99. Roberts BW, Kilgannon JH, Chansky ME, Mittal N, Wooden J, Trzeciak S (2013) Association between postresuscitation partial pressure of arterial carbon dioxide and neurological outcome in patients with post-cardiac arrest syndrome. Circulation 127:2107-2113

100. Wang HE, Prince DK, Drennan IR et al (2017) Post-resuscitation arterial oxygen and carbon dioxide and outcomes after out-of-hospital cardiac arrest. Resuscitation 120:113-118

101. von Auenmueller Kl, Christ M, Sasko BM, Trappe HJ (2017) The value of arterial blood gas parameters for prediction of mortality in survivors of out-of-hospital cardiac arrest. J Emerg Trauma Shock 10:134-139

102. Ebner F, Harmon MBA, Aneman A et al (2018) Carbon dioxide dynamics in relation to neurological outcome in resuscitated out-of-hospital cardiac arrest patients: an exploratory target temperature management trial substudy. Crit Care 22:196

103. McGuigan PJ, Shankar-Hari M, Harrison DA, Laffey JG, McAuley DF (2020) The interaction between arterial oxygenation and carbon dioxide and hospital mortality following out of hospital cardiac arrest: a cohort study. Crit Care 24:336

104. Falkenbach P, Kamarainen A, Makela A et al (2009) Incidence of iatrogenic dyscarbia during mild therapeutic hypothermia after successful resuscitation from out-of-hospital cardiac arrest. Resuscitation 80:990-993

105. Eastwood GM, Nielsen N, Nichol AD, Skrifvars MB, French C, Bellomo R (2019) Reported practice of temperature adjustment (alpha-stat $v$ pHstat) for arterial blood gases measurement among investigators from two major cardiac arrest trials. Crit Care Resusc 21:69-71

106. Hoedemaekers C, van der Hoeven JG (2014) Is alpha-stat or pH-stat the best strategy during hypothermia after cardiac arrest? Crit Care Med 42:1950-1951

107. Griffiths MJD, McAuley DF, Perkins GD et al (2019) Guidelines on the management of acute respiratory distress syndrome. BMJ Open Respir Res 6:e000420
108. Beitler JR, Ghafouri TB, Jinadasa SP et al (2017) Favorable neurocognitive outcome with low tidal volume ventilation after cardiac arrest. Am J Respir Crit Care Med 195:1198-1206

109. Geri G, Passouant O, Dumas F et al (2017) Etiological diagnoses of outof-hospital cardiac arrest survivors admitted to the intensive care unit: insights from a French registry. Resuscitation 117:66-72

110. Vahatalo JH, Huikuri HV, Holmstrom LTA et al (2019) Association of silent myocardial infarction and sudden cardiac death. JAMA Cardiol 4:796-802

111. Patterson T, Perkins GD, Hassan Y et al (2018) Temporal trends in identification, management, and clinical outcomes after out-of-hospital cardiac arrest: insights from the myocardial ischaemia national audit project database. Circ Cardiovasc Interv 11:e005346

112. Nikolaou NI, Welsford M, Beygui F et al (2015) Part 5: acute coronary syndromes: 2015 international consensus on cardiopulmonary resuscitation and emergency cardiovascular care science with treatment recommendations. Resuscitation 95:e121-146

113. Ibanez B, James S, Agewall S et al (2018) 2017 ESC Guidelines for the management of acute myocardial infarction in patients presenting with ST-segment elevation: the Task Force for the management of acute myocardial infarction in patients presenting with ST-segment elevation of the European Society of Cardiology (ESC). Eur Heart J 39:1 19-177

114. Elfwen $L$, Lagedal $R$, James $S$ et al (2018) Coronary angiography in out-of-hospital cardiac arrest without ST elevation on ECG-Short- and long-term survival. Am Heart J 200:90-95

115. Dumas F, Bougouin W, Geri G et al (2016) Emergency percutaneous coronary intervention in post-cardiac arrest patients without ST-segment elevation pattern: insights from the PROCAT II registry. JACC CardiovasC Interv 9:1011-1018

116. Kern KB, Radsel P, Jentzer JC et al (2020) Randomized pilot clinical trial of early coronary angiography versus no early coronary angiography after cardiac arrest without ST-segment elevation: the PEARL study. Circulation 142:2002-2012

117. Bougouin W, Dumas F, Karam N et al (2018) Should we perform an immediate coronary angiogram in all patients after cardiac arrest? Insights From a Large French Registry. JACC Cardiovasc Interv 11:249-256

118. Oksanen T, Skrifvars M, Wilkman E, Tierala I, Pettila V, Varpula T (2014) Postresuscitation hemodynamics during therapeutic hypothermia after out-of-hospital cardiac arrest with ventricular fibrillation: a retrospective study. Resuscitation 85:1018-1024

119. Uray T, Lamade A, Elmer J et al (2018) Phenotyping cardiac arrest: bench and bedside characterization of brain and heart injury based on etiology. Crit Care Med 46:e508-e515

120. Anderson RJ, Jinadasa SP, Hsu L et al (2018) Shock subtypes by left ventricular ejection fraction following out-of-hospital cardiac arrest. Crit Care 22:162

121. Grand J, Kjaergaard J, Bro-Jeppesen J et al (2019) Cardiac output, heart rate and stroke volume during targeted temperature management after out-of-hospital cardiac arrest: association with mortality and cause of death. Resuscitation 142:136-143

122. Soar J, Callaway CW, Aibiki M et al (2015) Part 4: Advanced life support: 2015 International Consensus on Cardiopulmonary Resuscitation and Emergency Cardiovascular Care Science with Treatment Recommendations. Resuscitation 95:e71-120

123. Trzeciak S, Jones AE, Kilgannon JH et al (2009) Significance of arterial hypotension after resuscitation from cardiac arrest. Crit Care Med 37:2895-2903

124. Kilgannon JH, Roberts BW, Stauss M et al (2008) Use of a standardized order set for achieving target temperature in the implementation of therapeutic hypothermia after cardiac arrest: a feasibility study. Acad Emerg Med 15:499-505

125. Gaieski DF, Band RA, Abella BS et al (2009) Early goal-directed hemodynamic optimization combined with therapeutic hypothermia in comatose survivors of out-of-hospital cardiac arrest. Resuscitation 80:418-424

126. Sunde K, Pytte M, Jacobsen D et al (2007) Implementation of a standardised treatment protocol for post resuscitation care after out-ofhospital cardiac arrest. Resuscitation 73:29-39

127. Mullner M, Sterz F, Binder M et al (1996) Arterial blood pressure after human cardiac arrest and neurological recovery. Stroke 27:59-62 
128. Walters EL, Morawski K, Dorotta I et al (2011) Implementation of a post-cardiac arrest care bundle including therapeutic hypothermia and hemodynamic optimization in comatose patients with return of spontaneous circulation after out-of-hospital cardiac arrest: a feasibility study. Shock 35:360-366

129. Kilgannon JH, Roberts BW, Jones AE et al (2014) Arterial blood pressure and neurologic outcome after resuscitation from cardiac arrest. Crit Care Med 42:2083-2091

130. Beylin ME, Perman SM, Abella BS et al (2013) Higher mean arterial pressure with or without vasoactive agents is associated with increased survival and better neurological outcomes in comatose survivors of cardiac arrest. Intensive Care Med 39:1981-1988

131. Ameloot K, De Deyne C, Eertmans W et al (2019) Early goal-directed haemodynamic optimization of cerebral oxygenation in comatose survivors after cardiac arrest: the Neuroprotect post-cardiac arrest trial. Eur Heart J 40:1804-1814

132. Jakkula P, Pettila V, Skrifvars MB et al (2018) Targeting low-normal or high-normal mean arterial pressure after cardiac arrest and resuscitation: a randomised pilot trial. Intensive Care Med 44:2091-2101

133. Ameloot K, Genbrugge C, Meex I et al (2015) An observational nearinfrared spectroscopy study on cerebral autoregulation in post-cardiac arrest patients: time to drop "one-size-fits-all" hemodynamic targets? Resuscitation 90:121-126

134. Ameloot K, Meex I, Genbrugge C et al (2015) Hemodynamic targets during therapeutic hypothermia after cardiac arrest: a prospective observational study. Resuscitation 91:56-62

135. Annoni F, Dell'Anna AM, Franchi F et al (2018) The impact of diastolic blood pressure values on the neurological outcome of cardiac arrest patients. Resuscitation 130:167-173

136. Bro-Jeppesen J, Annborn M, Hassager C et al (2015) Hemodynamics and vasopressor support during targeted temperature management at 33 degrees $C$ Versus $36^{\circ} \mathrm{C}$ after out-of-hospital cardiac arrest: a post hoc study of the target temperature management trial*. Crit Care Med 43:318-327

137. Chiu YK, Lui CT, Tsui KL (2018) Impact of hypotension after return of spontaneous circulation on survival in patients of out-of-hospital cardiac arrest. Am J Emerg Med 36:79-83

138. Huang $\mathrm{CH}$, Tsai MS, Ong HN et al (2017) Association of hemodynamic variables with in-hospital mortality and favorable neurological outcomes in post-cardiac arrest care with targeted temperature management. Resuscitation 120:146-152

139. Laurikkala J, Wilkman E, Pettila V et al (2016) Mean arterial pressure and vasopressor load after out-of-hospital cardiac arrest: associations with 1-year neurologic outcome. Resuscitation 105:116-122

140. Janiczek JA, Winger DG, Coppler P et al (2016) Hemodynamic resuscitation characteristics associated with improved survival and shock resolution after cardiac arrest. Shock 45:613-619

141. Russo JJ, Di Santo P, Simard T et al (2018) Optimal mean arterial pressure in comatose survivors of out-of-hospital cardiac arrest: an analysis of area below blood pressure thresholds. Resuscitation 128:175-180

142. Young MN, Hollenbeck RD, Pollock JS et al (2015) Higher achieved mean arterial pressure during therapeutic hypothermia is not associated with neurologically intact survival following cardiac arrest. Resuscitation 88:158-164

143. Scheinberg P, Jayne HW (1952) Factors influencing cerebral blood flow and metabolism; a review. Circulation 5:225-234

144. Haddad SH, Arabi YM (2012) Critical care management of severe traumatic brain injury in adults. Scand J Trauma Resusc Emerg Med 20:12

145. Sundgreen C, Larsen FS, Herzog TM, Knudsen GM, Boesgaard S, Aldershvile J (2001) Autoregulation of cerebral blood flow in patients resuscitated from cardiac arrest. Stroke 32:128-132

146. Sekhon MS, Griesdale DE (2017) Individualized perfusion targets in hypoxic ischemic brain injury after cardiac arrest. Crit Care 21:259

147. Sekhon MS, Gooderham P, Menon DK et al (2019) The Burden of brain hypoxia and optimal mean arterial pressure in patients with hypoxic ischemic brain injury after cardiac arrest. Crit Care Med 47:960-969

148. Hoiland RL, Robba C, Menon DK, Sekhon MS (2020) Differential pathophysiologic phenotypes of hypoxic ischemic brain injury: considerations for post-cardiac arrest trials. Intensive Care Med 46:1969-1971

149. van den Brule JM, Vinke E, van Loon LM, van der Hoeven JG, Hoedemaekers CW (2017) Middle cerebral artery flow, the critical closing pressure, and the optimal mean arterial pressure in comatose cardiac arrest survivors - an observational study. Resuscitation 110:85-89

150. Buunk G, van der Hoeven JG, Meinders AE (1997) Cerebrovascular reactivity in comatose patients resuscitated from a cardiac arrest. Stroke 28:1569-1573

151. Lemiale V, Huet O, Vigue B et al (2008) Changes in cerebral blood flow and oxygen extraction during post-resuscitation syndrome. Resuscitation 76:17-24

152. Rafi S, Tadie JM, Gacouin A et al (2019) Doppler sonography of cerebral blood flow for early prognostication after out-of-hospital cardiac arrest: DOTAC study. Resuscitation 141:188-194

153. Torgersen C, Meichtry J, Schmittinger CA et al (2013) Haemodynamic variables and functional outcome in hypothermic patients following out-of-hospital cardiac arrest. Resuscitation 84:798-804

154. Post H, Schmitto JD, Steendijk P et al (2010) Cardiac function during mild hypothermia in pigs: increased inotropy at the expense of diastolic dysfunction. Acta Physiol (Oxf) 199:43-52

155. Staer-Jensen H, Sunde K, Olasveengen TM et al (2014) Bradycardia during therapeutic hypothermia is associated with good neurologic outcome in comatose survivors of out-of-hospital cardiac arrest. Crit Care Med 42:2401-2408

156. Thomsen JH, Hassager C, Bro-Jeppesen J et al (2015) Sinus bradycardia during hypothermia in comatose survivors of out-of-hospital cardiac arrest-a new early marker of favorable outcome? Resuscitation 89:36-42

157. Oksanen T, Tiainen M, Vaahersalo J et al (2018) Lower heart rate is associated with good 1-year outcome in post-resuscitation patients. Resuscitation 128:112-118

158. Adler C, Reuter H, Seck C, Hellmich M, Zobel C (2013) Fluid therapy and acute kidney injury in cardiogenic shock after cardiac arrest. Resuscitation 84:194-199

159. Gamper G, Havel C, Arrich J et al (2016) Vasopressors for hypotensive shock. Cochrane Database Syst Rev 2:CD003709

160. Levy B, Clere-Jehl R, Legras A et al (2018) Epinephrine versus norepinephrine for cardiogenic shock after acute myocardial infarction. J Am Coll Cardiol 72:173-182

161. Kern KB, Hilwig RW, Berg RA et al (1997) Postresuscitation left ventricular systolic and diastolic dysfunction: treatment with dobutamine. Circulation 95:2610-2613

162. Vasquez A, Kern KB, Hilwig RW, Heidenreich J, Berg RA, Ewy GA (2004) Optimal dosing of dobutamine for treating post-resuscitation left ventricular dysfunction. Resuscitation 61:199-207

163. Mentzelopoulos SD, Malachias S, Chamos C et al (2013) Vasopressin, steroids, and epinephrine and neurologically favorable survival after in-hospital cardiac arrest: a randomized clinical trial. JAMA 310:270-279

164. Mentzelopoulos SD, Zakynthinos SG, Tzoufi M et al (2009) Vasopressin, epinephrine, and corticosteroids for in-hospital cardiac arrest. Arch Intern Med 169:15-24

165. Tsai MS, Chuang PY, Huang CH et al (2019) Postarrest steroid use may improve outcomes of cardiac arrest survivors. Crit Care Med 47:167-175

166. Donnino MW, Andersen LW, Berg KM et al (2016) Corticosteroid therapy in refractory shock following cardiac arrest: a randomized, double-blind, placebo-controlled, trial. Crit Care 20:82

167. Deakin CD, Morrison LJ, Morley PT et al (2010) Part 8: advanced life support: 2010 international consensus on cardiopulmonary resuscitation and emergency cardiovascular care science with treatment recommendations. Resuscitation 81(Suppl 1):e93-e174

168. Skrifvars MB, Pettila V, Rosenberg PH, Castren M (2003) A multiple logistic regression analysis of in-hospital factors related to survival at 6 months in patients resuscitated from out-of-hospital ventricular fibrillation. Resuscitation 59:319-328

169. Manzo-Silberman S, Fichet J, Mathonnet A et al (2013) Percutaneous left ventricular assistance in post cardiac arrest shock: comparison of intra aortic blood pump and IMPELLA Recover LP2.5. Resuscitation 84:609-615

170. O'Neill BP, Cohen MG, Basir MB et al (2019) Outcomes among patients transferred for revascularization with impella for acute myocardial infarction with cardiogenic shock from the CVAD registry. Am J Cardiol 123:1214-1219 
171. Ostenfeld S, Lindholm MG, Kjaergaard J et al (2015) Prognostic implication of out-of-hospital cardiac arrest in patients with cardiogenic shock and acute myocardial infarction. Resuscitation 87:57-62

172. Thiele H, Zeymer U, Neumann FJ et al (2012) Intraaortic balloon support for myocardial infarction with cardiogenic shock. N Engl J Med 367:1287-1296

173. Ahmad Y, Sen S, Shun-Shin MJ et al (2015) Intra-aortic Balloon pump therapy for acute myocardial infarction: a meta-analysis. JAMA Intern Med 175:931-939

174. Ouweneel DM, Eriksen E, Sjauw KD et al (2017) Percutaneous mechanical circulatory support versus intra-aortic balloon pump in cardiogenic shock after acute myocardial infarction. J Am Coll Cardiol 69:278-287

175. Priori SG, Blomstrom-Lundqvist C, Mazzanti A et al (2015) 2015 ESC Guidelines for the management of patients with ventricular arrhythmias and the prevention of sudden cardiac death: the Task Force for the Management of Patients with Ventricular Arrhythmias and the Prevention of Sudden Cardiac Death of the European Society of Cardiology (ESC). Endorsed by: Association for European Paediatric and Congenital Cardiology (AEPC). Eur Heart J 36:2793-2867

176. Maron MS, Rowin EJ, Wessler BS et al (2019) Enhanced American College of Cardiology/American Heart Association strategy for prevention of sudden cardiac death in high-risk patients with hypertrophic cardiomyopathy. JAMA Cardiol 4:644-657

177. Alba AC, Foroutan F, Duero Posada J et al (2018) Implantable cardiac defibrillator and mortality in non-ischaemic cardiomyopathy: an updated meta-analysis. Heart 104:230-236

178. Lybeck A, Friberg H, Aneman A et al (2017) Prognostic significance of clinical seizures after cardiac arrest and target temperature management. Resuscitation 114:146-151

179. Seder DB, Sunde K, Rubertsson S et al (2015) Neurologic outcomes and postresuscitation care of patients with myoclonus following cardiac arrest. Crit Care Med 43:965-972

180. Gupta HV, Caviness JN (2016) Post-hypoxic myoclonus: current concepts, neurophysiology, and treatment. Tremor Other Hyperkinet Mov (NY) 6:409

181. Elmer J, Rittenberger JC, Faro J et al (2016) Clinically distinct electroencephalographic phenotypes of early myoclonus after cardiac arrest. Ann Neurol 80:175-184

182. Lucas JM, Cocchi MN, Salciccioli J et al (2012) Neurologic recovery after therapeutic hypothermia in patients with post-cardiac arrest myoclonus. Resuscitation 83:265-269

183. Bouwes A, van Poppelen D, Koelman JH et al (2012) Acute posthypoxic myoclonus after cardiopulmonary resuscitation. BMC Neurol 12:63

184. Aicua Rapun I, Novy J, Solari D, Oddo M, Rossetti AO (2017) Early LanceAdams syndrome after cardiac arrest: prevalence, time to return to awareness, and outcome in a large cohort. Resuscitation 115:169-172

185. Lance JW, Adams RD (1963) The syndrome of intention or action myoclonus as a sequel to hypoxic encephalopathy. Brain 86:111-136

186. Backman S, Westhall E, Dragancea I et al (2017) Electroencephalographic characteristics of status epilepticus after cardiac arrest. Clin Neurophysiol 128:681-688

187. Hirsch L, Fong MWK, Leitinger M et al (2021) American Clinical Neurophysiology Society's Standardized Critical Care EEG Terminology: 2021 Version. J Clin Neurophysiol 38:1-29

188. Koutroumanidis M, Sakellariou D (2015) Low frequency nonevolving generalized periodic epileptiform discharges and the borderland of hypoxic nonconvulsive status epilepticus in comatose patients after cardiac arrest. Epilepsy Behav 49:255-262

189. Thomke F, Weilemann SL (2010) Poor prognosis despite successful treatment of postanoxic generalized myoclonus. Neurology 74:1392-1394

190. Solanki P, Coppler PJ, Kvaloy JT et al (2019) Association of antiepileptic drugs with resolution of epileptiform activity after cardiac arrest. Resuscitation 142:82-90

191. Dijk JM, Tijssen MA (2010) Management of patients with myoclonus: available therapies and the need for an evidence-based approach. Lancet Neurol 9:1028-1036

192. Zaccara G, Giorgi FS, Amantini A et al (2018) Why we prefer levetiracetam over phenytoin for treatment of status epilepticus. Acta Neuro Scand 137:618-622
193. Brain Resuscitation Clinical Trial I Study Group (1986) Randomized clinical study of thiopental loading in comatose survivors of cardiac arrest. N Engl J Med 314:397-403

194. Longstreth WT Jr, Fahrenbruch CE, Olsufka M, Walsh TR, Copass MK, Cobb LA (2002) Randomized clinical trial of magnesium, diazepam, or both after out-of-hospital cardiac arrest. Neurology 59:506-514

195. Ruijter BJ, van Putten MJ, Horn J et al (2014) Treatment of electroencephalographic status epilepticus after cardiopulmonary resuscitation (TELSTAR): study protocol for a randomized controlled trial. Trials 15:433

196. Beretta S, Coppo A, Bianchi E et al (2018) Neurologic outcome of postanoxic refractory status epilepticus after aggressive treatment. Neurology 91:e2153-e2162

197. Dragancea I, Backman S, Westhall E, Rundgren M, Friberg H, Cronberg $T$ (2015) Outcome following postanoxic status epilepticus in patients with targeted temperature management after cardiac arrest. Epilepsy Behav 49:173-177

198. Hofmeijer J, Tjepkema-Cloostermans MC, Blans MJ, Beishuizen A, van Putten MJ (2014) Unstandardized treatment of electroencephalographic status epilepticus does not improve outcome of comatose patients after cardiac arrest. Front Neurol 5:39

199. Rossetti AO, Oddo M, Liaudet L, Kaplan PW (2009) Predictors of awakening from postanoxic status epilepticus after therapeutic hypothermia. Neurology 72:744-749

200. Crepeau AZ, Fugate JE, Mandrekar J et al (2014) Value analysis of continuous EEG in patients during therapeutic hypothermia after cardiac arrest. Resuscitation 85:785-789

201. Sondag L, Ruijter BJ, Tjepkema-Cloostermans MC et al (2017) Early EEG for outcome prediction of postanoxic coma: prospective cohort study with cost-minimization analysis. Crit Care 21:111

202. Dragancea I, Horn J, Kuiper M et al (2015) Neurological prognostication after cardiac arrest and targeted temperature management $33^{\circ} \mathrm{C}$ versus $36^{\circ} \mathrm{C}$ : results from a randomised controlled clinical trial. Resuscitation 93:164-170

203. Barbella G, Lee JW, Alvarez V et al (2020) Prediction of regaining consciousness despite an early epileptiform EEG after cardiac arrest. Neurology 94:e1675-e1683

204. Rey A, Rossetti AO, Miroz JP, Eckert P, Oddo M (2019) Late awakening in survivors of postanoxic coma: early neurophysiologic predictors and association with ICU and long-term neurologic recovery. Crit Care Med 47:85-92

205. Donnino MW, Andersen LW, Berg KM et al (2016) Temperature management after cardiac arrest: an advisory statement by the advanced life support task force of the International Liaison Committee on Resuscitation and the American Heart Association Emergency Cardiovascular Care Committee and the Council on Cardiopulmonary, Critical Care, Perioperative and Resuscitation. Resuscitation 98:97-104

206. Donnino MW, Andersen LW, Berg KM et al (2015) Temperature management after cardiac arrest: an advisory statement by the advanced life support task force of the international liaison committee on resuscitation and the American Heart Association Emergency Cardiovascular Care Committee and the Council on Cardiopulmonary, Critical Care, Perioperative and Resuscitation. Circulation 132:2448-2456

207. Callaway CW, Soar J, Aibiki M et al (2015) Part 4: advanced life support: 2015 international consensus on cardiopulmonary resuscitation and emergency cardiovascular care science with treatment recommendations. Circulation 132:S84-145

208. Obermeyer Z, Samra JK, Mullainathan S (2017) Individual differences in normal body temperature: longitudinal big data analysis of patient records. BMJ 359:55468

209. Coppler PJ, Marill KA, Okonkwo DO et al (2016) Concordance of brain and core temperature in comatose patients after cardiac arrest. Ther Hypothermia Temp Manag 6:194-197

210. Zeiner A, Holzer M, Sterz F et al (2001) Hyperthermia after cardiac arrest is associated with an unfavorable neurologic outcome. Arch Intern Med 161:2007-2012

211. Makker P, Kanei Y, Misra D (2017) Clinical effect of rebound hyperthermia after cooling postcardiac arrest: a meta-analysis. Ther Hypothermia Temp Manag 7:206-209

212. Picetti E, Antonini MV, Bartolini Y et al (2016) Delayed fever and neurological outcome after cardiac arrest: a retrospective clinical study. Neurocrit Care 24:163-171 
213. Olai H, Thorneus $G$, Watson $H$ et al (2020) Meta-analysis of targeted temperature management in animal models of cardiac arrest. Intensive Care Med Exp 8:3

214. Drury PP, Gunn ER, Bennet L, Gunn AJ (2014) Mechanisms of hypothermic neuroprotection. Clin Perinatol 41:161-175

215. McCullough JN, Zhang N, Reich DL et al (1999) Cerebral metabolic suppression during hypothermic circulatory arrest in humans. Ann Thorac Surg 67:1895-1899

216. Gunn AJ, Thoresen M (2006) Hypothermic neuroprotection. NeuroRx 3:154-169

217. Bro-Jeppesen J, Kjaergaard J, Wanscher M et al (2014) The inflammatory response after out-of-hospital cardiac arrest is not modified by targeted temperature management at 33 degrees $C$ or 36 degrees $C$. Resuscitation 85:1480-1487

218. Hypothermia after Cardiac Arrest Study G (2002) Mild therapeutic hypothermia to improve the neurologic outcome after cardiac arrest. $\mathrm{N}$ Engl J Med 346:549-556

219. Bernard SA, Gray TW, Buist MD et al (2002) Treatment of comatose survivors of out-of-hospital cardiac arrest with induced hypothermia. N Engl J Med 346:557-563

220. Mader TJ, Nathanson BH, Soares WE 3rd, Coute RA, McNally BF (2014) Comparative effectiveness of therapeutic hypothermia after out-ofhospital cardiac arrest: insight from a large data registry. Ther Hypothermia Temp Manag 4:21-31

221. Marion DW, Leonov Y, Ginsberg M et al (1996) Resuscitative hypothermia. Crit Care Med 24:S81-89

222. Bernard SA, Smith K, Cameron P et al (2010) Induction of therapeutic hypothermia by paramedics after resuscitation from out-of-hospital ventricular fibrillation cardiac arrest: a randomized controlled trial. Circulation 122:737-742

223. Kim F, Nichol G, Maynard C et al (2014) Effect of prehospital induction of mild hypothermia on survival and neurological status among adults with cardiac arrest: a randomized clinical trial. JAMA 311:45-52

224. Bernard SA, Smith K, Finn J et al (2016) Induction of therapeutic hypothermia during out-of-hospital cardiac arrest using a rapid infusion of cold saline: the RINSE Trial (Rapid Infusion of Cold Normal Saline). Circulation 134:797-805

225. Castren M, Nordberg P, Svensson L et al (2010) Intra-arrest transnasal evaporative cooling: a randomized, prehospital, multicenter study (PRINCE: Pre-ROSC IntraNasal Cooling Effectiveness). Circulation 122:729-736

226. Nordberg P, Taccone FS, Truhlar A et al (2019) Effect of trans-nasal evaporative intra-arrest cooling on functional neurologic outcome in out-of-hospital cardiac arrest: the PRINCESS randomized clinical trial. JAMA 321:1677-1685

227. Awad A, Taccone FS, Jonsson M et al (2020) Time to intra-arrest therapeutic hypothermia in out-of-hospital cardiac arrest patients and its association with neurologic outcome: a propensity matched subanalysis of the PRINCESS trial. Intensive Care Med 46:1361-1370

228. Cronberg T, Lilja G, Horn J et al (2015) Neurologic function and healthrelated quality of life in patients following targeted temperature management at $33^{\circ} \mathrm{C}$ vs $36^{\circ} \mathrm{C}$ after out-of-hospital cardiac arrest: a randomized clinical trial. JAMA Neurol 72:634-641

229. Lilja G, Nielsen N, Friberg H et al (2015) Cognitive function in survivors of out-of-hospital cardiac arrest after target temperature management at $33^{\circ} \mathrm{C}$ versus $36^{\circ} \mathrm{C}$. Circulation 131:1340-1349

230. Stammet P, Collignon O, Hassager C et al (2015) Neuron-specific enolase as a predictor of death or poor neurological outcome after out-of-hospital cardiac arrest and targeted temperature management at $33^{\circ} \mathrm{C}$ and $36^{\circ} \mathrm{C}$. J Am Coll Cardiol 65:2104-2114

231. Moseby-Knappe M, Mattsson N, Nielsen N et al (2019) Serum neurofilament light chain for prognosis of outcome after cardiac arrest. JAMA Neurol 76:64-71

232. Annborn M, Bro-Jeppesen J, Nielsen $\mathrm{N}$ et al (2014) The association of targeted temperature management at 33 and $36^{\circ} \mathrm{C}$ with outcome in patients with moderate shock on admission after out-of-hospital cardiac arrest: a post hoc analysis of the target temperature management trial. Intensive Care Med 40:1210-1219

233. Lopez-de-Sa E, Juarez M, Armada E et al (2018) A multicentre randomized pilot trial on the effectiveness of different levels of cooling in comatose survivors of out-of-hospital cardiac arrest: the FROST-I trial. Intensive Care Med 44:1807-1815

234. Deye N, Vincent F, Michel P et al (2016) Changes in cardiac arrest patients' temperature management after the 2013 "TTM" trial: results from an international survey. Ann Intensive Care 6:4

235. Storm C, Nee J, Sunde K et al (2017) A survey on general and temperature management of post cardiac arrest patients in large teaching and university hospitals in 14 European countries - the SPAME trial results. Resuscitation 116:84-90

236. Bray JE, Stub D, Bloom JE et al (2017) Changing target temperature from $33{ }^{\circ} \mathrm{C}$ to $36^{\circ} \mathrm{C}$ in the ICU management of out-of-hospital cardiac arrest: a before and after study. Resuscitation 113:39-43

237. Casamento A, Minson A, Radford S et al (2016) A comparison of therapeutic hypothermia and strict therapeutic normothermia after cardiac arrest. Resuscitation 106:83-88

238. Bradley SM, Liu W, McNally B et al (2018) Temporal trends in the use of therapeutic hypothermia for out-of-hospital cardiac arrest. JAMA Netw Open 1:e184511

239. Salter R, Bailey M, Bellomo R et al (2018) Changes in temperature management of cardiac arrest patients following publication of the target temperature management trial. Crit Care Med 46:1722-1730

240. Nielsen N, Friberg H (2015) Temperature management after cardiac arrest. Curr Opin Crit Care 21:202-208

241. Yokoyama H, Nagao K, Hase M et al (2011) Impact of therapeutic hypothermia in the treatment of patients with out-of-hospital cardiac arrest from the J-PULSE-HYPO study registry. Circ J 75:1063-1070

242. Lee BK, Lee SJ, Jeung KW, Lee HY, Heo T, Min YI (2014) Outcome and adverse events with 72 -h cooling at $32{ }^{\circ} \mathrm{C}$ as compared to $24-\mathrm{h}$ cooling at $33^{\circ} \mathrm{C}$ in comatose asphyxial arrest survivors. Am J Emerg Med 32:297-301

243. Kirkegaard H, Soreide E, de Haas I et al (2017) Targeted temperature management for 48 vs $24 \mathrm{~h}$ and neurologic outcome after out-ofhospital cardiac arrest: a randomized clinical trial. JAMA 318:341-350

244. Damian MS, Ellenberg D, Gildemeister R et al (2004) Coenzyme Q10 combined with mild hypothermia after cardiac arrest: a preliminary study. Circulation 110:3011-3016

245. Grafton ST, Longstreth WT Jr (1988) Steroids after cardiac arrest: a retrospective study with concurrent, nonrandomized controls. Neurology 38:1315-1316

246. Gueugniaud PY, Gaussorgues P, Garcia-Darennes F et al (1990) Early effects of nimodipine on intracranial and cerebral perfusion pressures in cerebral anoxia after out-of-hospital cardiac arrest. Resuscitation 20:203-212

247. Roine RO, Kaste M, Kinnunen A, Nikki P, Sarna S, Kajaste S (1990) Nimodipine after resuscitation from out-of-hospital ventricular fibrillation: a placebo-controlled, double-blind, randomized trial. JAMA 264:3171-3177

248. Cariou A, Deye N, Vivien B et al (2016) Early high-dose erythropoietin therapy after out-of-hospital cardiac arrest: a multicenter, randomized controlled trial. J Am Coll Cardiol 68:40-49

249. Argaud L, Cour M, Dubien PY et al (2016) Effect of cyclosporine in nonshockable out-of-hospital cardiac arrest: the CYRUS randomized clinical trial. JAMA Cardiol 1:557-565

250. Wiberg S, Hassager C, Schmidt H et al (2016) Neuroprotective effects of the glucagon-like peptide-1 analog exenatide after out-of-hospital cardiac arrest: a randomized controlled trial. Circulation 134:2115-2124

251. Thoresen M, Hobbs CE, Wood T, Chakkarapani E, Dingley J (2009) Cooling combined with immediate or delayed xenon inhalation provides equivalent long-term neuroprotection after neonatal hypoxia-ischemia. I Cereb Blood Flow Metab 29:707-714

252. Arola OJ, Laitio RM, Roine RO et al (2013) Feasibility and cardiac safety of inhaled xenon in combination with therapeutic hypothermia following out-of-hospital cardiac arrest. Crit Care Med 41:2116-2124

253. Arola O, Saraste A, Laitio R et al (2017) Inhaled xenon attenuates myocardial damage in comatose survivors of out-of-hospital cardiac arrest: The Xe-Hypotheca trial. J Am Coll Cardiol 70:2652-2660

254. Laitio R, Hynninen M, Arola O et al (2016) Effect of inhaled xenon on cerebral white matter damage in comatose survivors of out-of-hospital cardiac arrest: a randomized clinical trial. JAMA 315:1120-1128

255. Knapp J, Bergmann G, Bruckner T, Russ N, Bottiger BW, Popp E (2013) Pre- and postconditioning effect of Sevoflurane on myocardial 
dysfunction after cardiopulmonary resuscitation in rats. Resuscitation 84:1450-1455

256. Soukup J, Selle A, Wienke A, Steighardt J, Wagner NM, Kellner P (2012) Efficiency and safety of inhalative sedation with sevoflurane in comparison to an intravenous sedation concept with propofol in intensive care patients: study protocol for a randomized controlled trial. Trials 13:135

257. Krannich A, Leithner C, Engels M et al (2017) Isoflurane sedation on the ICU in cardiac arrest patients treated with targeted temperature management: an observational propensity-matched study. Crit Care Med 45:e384-e390

258. Hellstrom J, Owall A, Martling CR, Sackey PV (2014) Inhaled isoflurane sedation during therapeutic hypothermia after cardiac arrest: a case series. Crit Care Med 42:e161-166

259. Vrselja Z, Daniele SG, Silbereis J et al (2019) Restoration of brain circulation and cellular functions hours post-mortem. Nature 568:336-343

260. Taunyane IC, Benk C, Beyersdorf F et al (2016) Preserved brain morphology after controlled automated reperfusion of the whole body following normothermic circulatory arrest time of up to 20 minutes. Eur J Cardiothorac Surg 50:1025-1034

261. Trummer G, Benk C, Beyersdorf F (2019) Controlled automated reperfusion of the whole body after cardiac arrest. J Thorac Dis 11:S1464-S1470

262. Trummer G, Supady A, Beyersdorf F et al (2017) Controlled automated reperfusion of the whole body after 120 min of Cardiopulmonary resuscitation: first clinical report. Scand J Trauma Resusc Emerg Med 25:66

263. Couper K, Laloo R, Field R, Perkins GD, Thomas M, Yeung J (2019) Prophylactic antibiotic use following cardiac arrest: a systematic review and meta-analysis. Resuscitation 141:166-173

264. Bjelland TW, Dale O, Kaisen K et al (2012) Propofol and remifentanil versus midazolam and fentanyl for sedation during therapeutic hypothermia after cardiac arrest: a randomised trial. Intensive Care Med 38:959-967

265. Paul M, Bougouin W, Dumas F et al (2018) Comparison of two sedation regimens during targeted temperature management after cardiac arrest. Resuscitation 128:204-210

266. Lascarrou JB, Le Gouge A, Dimet J et al (2014) Neuromuscular blockade during therapeutic hypothermia after cardiac arrest: observational study of neurological and infectious outcomes. Resuscitation 85:1257-1262

267. May TL, Riker RR, Fraser GL et al (2018) Variation in sedation and neuromuscular blockade regimens on outcome after cardiac arrest. Crit Care Med 46:e975-e980

268. Lee BK, Cho IS, Oh JS et al (2018) Continuous neuromuscular blockade infusion for out-of-hospital cardiac arrest patients treated with targeted temperature management: a multicenter randomized controlled trial. PLoS One 13:e0209327

269. Alhazzani W, Alshahrani M, Jaeschke R et al (2013) Neuromuscular blocking agents in acute respiratory distress syndrome: a systematic review and meta-analysis of randomized controlled trials. Crit Care 17:R43

270. Perbet S, Mongardon N, Dumas F et al (2011) Early-onset pneumonia after cardiac arrest: characteristics, risk factors and influence on prognosis. Am J Respir Crit Care Med 184:1048-1054

271. Francois B, Cariou A, Clere-Jehl R et al (2019) Prevention of early ventilator-associated pneumonia after cardiac arrest. N Engl J Med 381:1831-1842

272. Williams ML, Nolan JP (2014) Is enteral feeding tolerated during therapeutic hypothermia? Resuscitation 85:1469-1472

273. Krag M, Marker S, Perner A et al (2018) Pantoprazole in patients at risk for gastrointestinal bleeding in the ICU. N Engl J Med 379:2199-2208

274. Cook D, Guyatt G (2018) Prophylaxis against upper gastrointestinal bleeding in hospitalized patients. N Engl J Med 378:2506-2516

275. Wang Y, Ge L, Ye Z et al (2020) Efficacy and safety of gastrointestinal bleeding prophylaxis in critically ill patients: an updated systematic review and network meta-analysis of randomized trials. Intensive Care Med 46:1987-2000

276. Gianforcaro A, Kurz M, Guyette FX et al (2017) Association of antiplatelet therapy with patient outcomes after out-of-hospital cardiac arrest. Resuscitation 121:98-103
277. Schunemann HJ, Cushman M, Burnett AE et al (2018) American Society of Hematology 2018 guidelines for management of venous thromboembolism: prophylaxis for hospitalized and nonhospitalized medical patients. Blood Adv 2:3198-3225

278. Duranteau J, Taccone FS, Verhamme P, Ageno W, Force EVGT (2018) European guidelines on perioperative venous thromboembolism prophylaxis: intensive care. Eur J Anaesthesiol 35:142-146

279. Llau JV, Kamphuisen P, Albaladejo P, Force EVGT (2018) European guidelines on perioperative venous thromboembolism prophylaxis: chronic treatments with antiplatelet agents. Eur J Anaesthesiol 35:139-141

280. Van Poucke S, Stevens K, Marcus AE, Lance M (2014) Hypothermia: effects on platelet function and hemostasis. Thromb J 12:31

281. Andremont O, du Cheyron D, Terzi N et al (2018) Endovascular cooling versus standard femoral catheters and intravascular complications: a propensity-matched cohort study. Resuscitation 124:1-6

282. American DA (2019) 15. Diabetes Care in the Hospital: standards of medical care in diabetes-2019. Diabetes Care 42:S173-S181

283. Oksanen T, Skrifvars MB, Varpula T et al (2007) Strict versus moderate glucose control after resuscitation from ventricular fibrillation. Intensive Care Med 33:2093-2100

284. Investigators N-SS, Finfer S, Liu B et al (2012) Hypoglycemia and risk of death in critically ill patients. N Engl J Med 367:1108-1118

285. Oddo M, Poole D, Helbok R et al (2018) Fluid therapy in neurointensive care patients: ESICM consensus and clinical practice recommendations. Intensive Care Med 44:449-463

286. Sandroni C, D'Arrigo S, Callaway CW et al (2016) The rate of brain death and organ donation in patients resuscitated from cardiac arrest: a systematic review and meta-analysis. Intensive Care Med 42:1661-1671

287. Sandroni C, Cariou A, Cavallaro F et al (2014) Prognostication in comatose survivors of cardiac arrest: an advisory statement from the European Resuscitation Council and the European Society of Intensive Care Medicine. Intensive Care Med 40:1816-1831

288. Brain Resuscitation Clinical Trial 1 Study Group (1986) A randomized clinical study of cardiopulmonary-cerebral resuscitation: design, methods, and patient characteristics. Brain Resuscitation Clinical Trial I Study Group. Am J Emerg Med 4:72-86

289. Rankin J (1957) Cerebral vascular accidents in patients over the age of 60. II. Prognosis. Scott Med J 2:200-215

290. Haywood K, Whitehead L, Nadkarni VM et al (2018) COSCA (Core Outcome Set for Cardiac Arrest) in Adults: an Advisory Statement From the International Liaison Committee on Resuscitation. Resuscitation 127:147-163

291. Banks JL, Marotta CA (2007) Outcomes validity and reliability of the modified Rankin scale: implications for stroke clinical trials: a literature review and synthesis. Stroke 38:1091-1096

292. Raina KD, Callaway C, Rittenberger JC, Holm MB (2008) Neurological and functional status following cardiac arrest: method and tool utility. Resuscitation 79:249-256

293. Quinn TJ, Dawson J, Walters MR, Lees KR (2007) Reliability of the modified Rankin Scale. Stroke 38:e144

294. Sandroni C, Nolan JP (2015) Neuroprognostication after cardiac arrest in Europe: new timings and standards. Resuscitation 90:A4-5

295. Geocadin RG, Callaway CW, Fink EL et al (2019) Standards for studies of neurological prognostication in comatose survivors of cardiac arrest: a scientific statement from the American Heart Association. Circulation 140:e517-e542

296. Steinberg A, Callaway CW, Arnold RM et al (2019) Prognostication after cardiac arrest: results of an international, multi-professional survey. Resuscitation 138:190-197

297. Sandroni C, Cariou A, Cavallaro F et al (2014) Prognostication in comatose survivors of cardiac arrest: an advisory statement from the European Resuscitation Council and the European Society of Intensive Care Medicine. Resuscitation 85:1779-1789

298. Sandroni C, Cavallaro F, Callaway CW et al (2013) Predictors of poor neurological outcome in adult comatose survivors of cardiac arrest: a systematic review and meta-analysis. Part 2: patients treated with therapeutic hypothermia. Resuscitation 84:1324-1338

299. Sandroni C, Cavallaro F, Callaway CW et al (2013) Predictors of poor neurological outcome in adult comatose survivors of cardiac arrest: a systematic review and meta-analysis. Part 1: patients not treated with therapeutic hypothermia. Resuscitation 84:1310-1323 
300. Scarpino M, Carrai R, Lolli F et al (2020) Neurophysiology for predicting good and poor neurological outcome at 12 and $72 \mathrm{~h}$ after cardiac arrest: the ProNeCA multicentre prospective study. Resuscitation 147:95-103

301. Scarpino M, Lanzo G, Lolli F et al (2018) Neurophysiological and neuroradiological multimodal approach for early poor outcome prediction after cardiac arrest. Resuscitation 129:114-120

302. Zhou SE, Maciel CB, Ormseth CH, Beekman R, Gilmore EJ, Greer DM (2019) Distinct predictive values of current neuroprognostic guidelines in post-cardiac arrest patients. Resuscitation 139:343-350

303. Dragancea I, Wise MP, Al-Subaie N et al (2017) Protocol-driven neurological prognostication and withdrawal of life-sustaining therapy after cardiac arrest and targeted temperature management. Resuscitation 117:50-57

304. Sandroni C, Dell'anna AM, Tujjar O, Geri G, Cariou A, Taccone FS (2016) Acute kidney injury after cardiac arrest: a systematic review and metaanalysis of clinical studies. Minerva Anestesiol 82:989-999

305. Paul M, Bougouin W, Geri G et al (2016) Delayed awakening after cardiac arrest: prevalence and risk factors in the Parisian registry. Intensive Care Med 42:1128-1136

306. Nobile L, Taccone FS, Szakmany T et al (2016) The impact of extracerebral organ failure on outcome of patients after cardiac arrest: an observational study from the ICON database. Crit Care 20:368

307. Taccone FS, Horn J, Storm C et al (2019) Death after awakening from post-anoxic coma: the "Best CPC" project. Crit Care 23:107

308. Olson DM, Stutzman S, Saju C, Wilson M, Zhao W, Aiyagari V (2016) Interrater reliability of pupillary assessments. Neurocrit Care 24:251-257

309. Solari D, Rossetti AO, Carteron L et al (2017) Early prediction of coma recovery after cardiac arrest with blinded pupillometry. Ann Neurol 81:804-810

310. Oddo M, Sandroni C, Citerio G et al (2018) Quantitative versus standard pupillary light reflex for early prognostication in comatose cardiac arrest patients: an international prospective multicenter doubleblinded study. Intensive Care Med 44:2102-2111

311. Wijdicks EF, Bamlet WR, Maramattom BV, Manno EM, McClelland RL (2005) Validation of a new coma scale: The FOUR score. Ann Neurol 58:585-593

312. Maciel CB, Barden MM, Youn TS, Dhakar MB, Greer DM (2020) Neuroprognostication practices in postcardiac arrest patients: an international survey of critical care providers. Crit Care Med 48:e107-e114

313. Moseby-Knappe M, Westhall E, Backman S et al (2020) Performance of a guideline-recommended algorithm for prognostication of poor neurological outcome after cardiac arrest. Intensive Care Med 46:1852-1862

314. van Zijl JC, Beudel M, vd Hoeven HJ, Lange F, Tijssen MA, Elting JW (2016) Electroencephalographic findings in posthypoxic myoclonus. J Intensive Care Med 31:270-275

315. Kongpolprom N, Cholkraisuwat J (2018) Neurological prognostications for the therapeutic hypothermia among comatose survivors of cardiac arrest. Indian J Crit Care Med 22:509-518

316. English WA, Giffin NJ, Nolan JP (2009) Myoclonus after cardiac arrest: pitfalls in diagnosis and prognosis. Anaesthesia 64:908-911

317. Ruknuddeen MI, Ramadoss R, Rajajee V, Grzeskowiak LE, Rajagopalan RE (2015) Early clinical prediction of neurological outcome following out of hospital cardiac arrest managed with therapeutic hypothermia. Indian J Crit Care Med 19:304-310

318. Friberg H, Cronberg T, Dunser MW, Duranteau J, Horn J, Oddo M (2015) Survey on current practices for neurological prognostication after cardiac arrest. Resuscitation 90:158-162

319. Westhall E, Rosen I, Rundgren M et al (2018) Time to epileptiform activity and EEG background recovery are independent predictors after cardiac arrest. Clin Neurophysiol 129:1660-1668

320. Cloostermans MC, van Meulen FB, Eertman CJ, Hom HW, van Putten MJ (2012) Continuous electroencephalography monitoring for early prediction of neurological outcome in postanoxic patients after cardiac arrest: a prospective cohort study. Crit Care Med 40:2867-2875

321. Rundgren M, Westhall E, Cronberg T, Rosen I, Friberg H (2010) Continuous amplitude-integrated electroencephalogram predicts outcome in hypothermia-treated cardiac arrest patients. Crit Care Med 38:1838-1844

322. Oh SH, Park KN, Shon YM et al (2015) Continuous amplitude-integrated electroencephalographic monitoring is a useful prognostic tool for hypothermia-treated cardiac arrest patients. Circulation 132:1094-1103

323. Jorgensen EO, Holm S (1998) The natural course of neurological recovery following cardiopulmonary resuscitation. Resuscitation 36:111-122

324. Drohan CM, Cardi Al, Rittenberger JC et al (2018) Effect of sedation on quantitative electroencephalography after cardiac arrest. Resuscitation 124:132-137

325. Ruijter BJ, van Putten M, van den Bergh WM, Tromp SC, Hofmeijer J (2019) Propofol does not affect the reliability of early EEG for outcome prediction of comatose patients after cardiac arrest. Clin Neurophysiol 130:1263-1270

326. Westhall E, Rossetti AO, van Rootselaar AF et al (2016) Standardized EEG interpretation accurately predicts prognosis after cardiac arrest. Neurology 86:1482-1490

327. Backman S, Cronberg T, Friberg H et al (2018) Highly malignant routine EEG predicts poor prognosis after cardiac arrest in the target temperature management trial. Resuscitation 131:24-28

328. Benarous L, Gavaret M, Soda Diop M et al (2019) Sources of interrater variability and prognostic value of standardized EEG features in postanoxic coma after resuscitated cardiac arrest. Clin Neurophysiol Pract $4: 20-26$

329. Caporro M, Rossetti AO, Seiler A et al (2019) Electromyographic reactivity measured with scalp-EEG contributes to prognostication after cardiac arrest. Resuscitation 138:146-152

330. Lamartine Monteiro M, Taccone FS, Depondt C et al (2016) The prognostic value of 48-h continuous EEG during therapeutic hypothermia after cardiac arrest. Neurocrit Care 24:153-162

331. Ruijter BJ, Tjepkema-Cloostermans MC, Tromp SC et al (2019) Early electroencephalography for outcome prediction of postanoxic coma: a prospective cohort study. Ann Neurol 86:203-214

332. Landis JR, Koch GG (1977) The measurement of observer agreement for categorical data. Biometrics 33:159-174

333. Hofmeijer J, Tjepkema-Cloostermans MC, van Putten MJ (2014) Burstsuppression with identical bursts: a distinct EEG pattern with poor outcome in postanoxic coma. Clin Neurophysiol 125:947-954

334. Amorim E, Rittenberger JC, Zheng JJ et al (2016) Continuous EEG monitoring enhances multimodal outcome prediction in hypoxic-ischemic brain injury. Resuscitation 109:121-126

335. Leao RN, Avila P, Cavaco R, Germano N, Bento L (2015) Therapeutic hypothermia after cardiac arrest: outcome predictors. Rev Bras Ter Intensiva 27:322-332

336. Duez CHV, Johnsen B, Ebbesen MQ et al (2019) Post resuscitation prognostication by EEG in 24 vs 48 h of targeted temperature management. Resuscitation 135:145-152

337. Noirhomme Q, Lehembre R, Lugo ZD et al (2014) Automated analysis of background EEG and reactivity during therapeutic hypothermia in comatose patients after cardiac arrest. Clin EEG Neurosci 45:6-13

338. Rossetti AO, Tovar Quiroga DF, Juan E et al (2017) Electroencephalography predicts poor and good outcomes after cardiac arrest: a two-center study. Crit Care Med 45:e674-e682

339. Admiraal MM, van Rootselaar AF, Horn J (2017) Electroencephalographic reactivity testing in unconscious patients: a systematic review of methods and definitions. Eur J Neurol 24:245-254

340. Admiraal MM, van Rootselaar AF, Hofmeijer J et al (2019) Electroencephalographic reactivity as predictor of neurological outcome in postanoxic coma: a multicenter prospective cohort study. Ann Neurol 86:17-27

341. Alvarez V, Reinsberger C, Scirica B et al (2015) Continuous electroderma activity as a potential novel neurophysiological biomarker of prognosis after cardiac arrest-a pilot study. Resuscitation 93:128-135

342. Grippo A, Carrai R, Scarpino M et al (2017) Neurophysiological prediction of neurological good and poor outcome in post-anoxic coma. Acta Neurol Scand 135:641-648

343. Fatuzzo D, Beuchat I, Alvarez V, Novy J, Oddo M, Rossetti AO (2018) Does continuous EEG influence prognosis in patients after cardiac arrest? Resuscitation 132:29-32

344. Liu G, Su Y, Liu Y et al (2016) Predicting outcome in comatose patients: the role of EEG reactivity to quantifiable electrical stimuli. Evid Based Complement Alternat Med 2016:8273716 
345. Sivaraju A, Gilmore EJ, Wira CR et al (2015) Prognostication of postcardiac arrest coma: early clinical and electroencephalographic predictors of outcome. Intensive Care Med 41:1264-1272

346. Westhall E, Rosen I, Rossetti AO et al (2015) Interrater variability of EEG interpretation in comatose cardiac arrest patients. Clin Neurophysiol 126:2397-2404

347. Alvarez V, Oddo M, Rossetti AO (2013) Stimulus-induced rhythmic, periodic or ictal discharges (SIRPIDs) in comatose survivors of cardiac arrest: characteristics and prognostic value. Clin Neurophysiol 124:204-208

348. Sadaka F, Doerr D, Hindia J, Lee KP, Logan W (2015) Continuous electroencephalogram in comatose postcardiac arrest syndrome patients treated with therapeutic hypothermia: outcome prediction study. J Intensive Care Med 30:292-296

349. Ruijter BJ, van Putten MJ, Hofmeijer J (2015) Generalized epileptiform discharges in postanoxic encephalopathy: quantitative characterization in relation to outcome. Epilepsia 56:1845-1854

350. De Santis P, Lamanna I, Mavroudakis N et al (2017) The potential role of auditory evoked potentials to assess prognosis in comatose survivors from cardiac arrest. Resuscitation 120:119-124

351. Amorim E, van der Stoel M, Nagaraj SB et al (2019) Quantitative EEG reactivity and machine learning for prognostication in hypoxicischemic brain injury. Clin Neurophysiol 130:1908-1916

352. Ruijter BJ, Hofmeijer J, Tjepkema-Cloostermans MC, van Putten M (2018) The prognostic value of discontinuous EEG patterns in postanoxic coma. Clin Neurophysiol 129:1534-1543

353. Nagaraj SB, Tjepkema-Cloostermans MC, Ruijter BJ, Hofmeijer J, van Putten M (2018) The revised Cerebral Recovery Index improves predictions of neurological outcome after cardiac arrest. Clin Neurophysiol 129:2557-2566

354. Eertmans W, Genbrugge C, Haesevoets G et al (2017) Recorded time periods of bispectral index values equal to zero predict neurological outcome after out-of-hospital cardiac arrest. Crit Care 21:221

355. Park JH, Oh JH, Choi SP, Wee JH (2018) Neurologic outcome after out-of-hospital cardiac arrest could be predicted with the help of bispectral-index during early targeted temperature management Scand J Trauma Resusc Emerg Med 26:59

356. Stammet P, Collignon O, Werer C, Sertznig C, Devaux Y (2014) Bispectral index to predict neurological outcome early after cardiac arrest. Resuscitation 85:1674-1680

357. Horn J, Tjepkema-Cloostermans MC (2017) Somatosensory evoked potentials in patients with hypoxic-ischemic brain injury. Semin Neurol 37:60-65

358. Choi SP, Park KN, Wee JH et al (2017) Can somatosensory and visual evoked potentials predict neurological outcome during targeted temperature management in post cardiac arrest patients? Resuscitation 119:70-75

359. Dhakal LP, Sen A, Stanko CM et al (2016) Early absent pupillary light reflexes after cardiac arrest in patients treated with therapeutic hypothermia. Ther Hypothermia Temp Manag 6:116-121

360. Hofmeijer J, Beernink TM, Bosch FH, Beishuizen A, Tjepkema-Cloostermans MC, van Putten MJ (2015) Early EEG contributes to multimodal outcome prediction of postanoxic coma. Neurology 85:137-143

361. Huntgeburth M, Adler C, Rosenkranz S et al (2014) Changes in neuron-specific enolase are more suitable than its absolute serum levels for the prediction of neurologic outcome in hypothermiatreated patients with out-of-hospital cardiac arrest. Neurocrit Care 20:358-366

362. Kim SW, Oh JS, Park J et al (2018) Short-latency positive peak following N20 somatosensory evoked potential is superior to N20 in predicting neurologic outcome after out-of-hospital cardiac arrest. Crit Care Med 46:e545-e551

363. Maciel CB, Morawo AO, Tsao CY et al (2017) SSEP in therapeutic hypothermia era. J Clin Neurophysiol 34:469-475

364. Maia B, Roque R, Amaral-Silva A, Lourenco S, Bento L, Alcantara J (2013) Predicting outcome after cardiopulmonary arrest in therapeutic hypothermia patients: clinical, electrophysiological and imaging prognosticators. Acta Med Port 26:93-97

365. Oddo M, Rossetti AO (2014) Early multimodal outcome prediction after cardiac arrest in patients treated with hypothermia. Crit Care Med 42:1340-1347
366. Tsetsou S, Novy J, Pfeiffer C, Oddo M, Rossetti AO (2018) Multimodal outcome prognostication after cardiac arrest and targeted temperature management: analysis at $36^{\circ} \mathrm{C}$. Neurocrit Care 28:104-109

367. Amorim E, Ghassemi MM, Lee JW et al (2018) Estimating the false positive rate of absent somatosensory evoked potentials in cardiac arrest prognostication. Crit Care Med 46:e1213-e1221

368. Pfeifer R, Weitzel S, Gunther A et al (2013) Investigation of the interobserver variability effect on the prognostic value of somatosensory evoked potentials of the median nerve (SSEP) in cardiac arrest survivors using an SSEP classification. Resuscitation 84:1375-1381

369. Zandbergen EG, Hijdra A, de Haan RJ et al (2006) Interobserver variation in the interpretation of SSEPS in anoxic-ischaemic coma. Clin Neurophysiol 117:1529-1535

370. Helwig K, Seeger F, Holschermann H et al (2017) Elevated serum glial fibrillary acidic protein (GFAP) is associated with poor functional outcome after cardiopulmonary resuscitation. Neurocrit Care 27:68-74

371. Mattsson N, Zetterberg H, Nielsen N et al (2017) Serum tau and neurological outcome in cardiac arrest. Ann Neurol 82:665-675

372. Rana OR, Schroder JW, Baukloh JK et al (2013) Neurofilament light chain as an early and sensitive predictor of long-term neurological outcome in patients after cardiac arrest. Int J Cardiol 168:1322-1327

373. Streitberger KJ, Leithner C, Wattenberg M et al (2017) Neuron-specific enolase predicts poor outcome after cardiac arrest and targeted temperature management: a multicenter study on 1053 patients. Crit Care Med 45:1145-1151

374. Vondrakova D, Kruger A, Janotka M et al (2017) Association of neuronspecific enolase values with outcomes in cardiac arrest survivors is dependent on the time of sample collection. Crit Care 21:172

375. Chung-Esaki HM, Mui G, Mlynash M, Eyngorn I, Catabay K, Hirsch KG (2018) The neuron specific enolase (NSE) ratio offers benefits over absolute value thresholds in post-cardiac arrest coma prognosis. J Clin Neurosci 57:99-104

376. Duez CHV, Grejs AM, Jeppesen AN et al (2018) Neuron-specific enolase and S-100 b in prolonged targeted temperature management after cardiac arrest: a randomised study. Resuscitation 122:79-86

377. Jang JH, Park WB, Lim YS et al (2019) Combination of S100B and procalcitonin improves prognostic performance compared to either alone in patients with cardiac arrest: a prospective observational study. Medicine (Baltimore) 98:e14496

378. Pfeifer R, Franz M, Figulla HR (2014) Hypothermia after cardiac arrest does not affect serum levels of neuron-specific enolase and protein S-100b. Acta Anaesthesiol Scand 58:1093-1100

379. Wiberg S, Hassager C, Stammet P et al (2017) Single versus serial measurements of neuron-specific enolase and prediction of poor neurological outcome in persistently unconscious patients after out-of-hospital cardiac arrest-a TTM-trial substudy. PLoS One 12:e0168894

380. Wihersaari L, Tiainen M, Skrifvars MB et al (2019) Usefulness of neuron specific enolase in prognostication after cardiac arrest: impact of age and time to ROSC. Resuscitation 139:214-221

381. Rundgren M, Cronberg T, Friberg H, Isaksson A (2014) Serum neuron specific enolase-impact of storage and measuring method. BMC Res Notes 7:726

382. Stammet P, Dankiewicz J, Nielsen N et al (2017) Protein S100 as outcome predictor after out-of-hospital cardiac arrest and targeted temperature management at $33^{\circ} \mathrm{C}$ and $36^{\circ} \mathrm{C}$. Crit Care 21:153

383. Wilson DH, Rissin DM, Kan CW et al (2016) The Simoa HD-1 analyzer: a novel fully automated digital immunoassay analyzer with singlemolecule sensitivity and multiplexing. J Lab Autom 21:533-547

384. Wihersaari L, Ashton NJ, Reinikainen M et al (2021) Neurofilament light as an outcome predictor after cardiac arrest: a post hoc analysis of the COMACARE trial. Intensive Care Med 47(1):39-48

385. Keijzer HM, Hoedemaekers CWE, Meijer FJA, Tonino BAR, Klijn CJM, Hofmeijer J (2018) Brain imaging in comatose survivors of cardiac arrest: pathophysiological correlates and prognostic properties. Resuscitation 133:124-136

386. Lee DH, Lee BK, Jeung KW et al (2018) Relationship between ventricular characteristics on brain computed tomography and 6-month neurologic outcome in cardiac arrest survivors who underwent targeted temperature management. Resuscitation 129:37-42

387. Scarpino M, Lolli F, Lanzo G et al (2019) Neurophysiology and neuroimaging accurately predict poor neurological outcome within $24 \mathrm{~h}$ 
after cardiac arrest: The ProNeCA prospective multicentre prognostication study. Resuscitation 143:115-123

388. Wang GN, Chen XF, Lv JR, Sun NN, Xu XQ, Zhang JS (2018) The prognostic value of gray-white matter ratio on brain computed tomography in adult comatose cardiac arrest survivors. J Chin Med Assoc 81:599-604

389. Kim JH, Kim MJ, You JS et al (2019) Multimodal approach for neurologic prognostication of out-of-hospital cardiac arrest patients undergoing targeted temperature management. Resuscitation 134:33-40

390. Lee BK, Kim WY, Shin J et al (2016) Prognostic value of gray matter to white matter ratio in hypoxic and non-hypoxic cardiac arrest with noncardiac etiology. Am J Emerg Med 34:1583-1588

391. Lee KS, Lee SE, Choi JY et al (2017) Useful computed tomography score for estimation of early neurologic outcome in post-cardiac arrest patients with therapeutic hypothermia. Circ J 81:1628-1635

392. Oh JH, Choi SP, Wee JH, Park JH (2019) Inter-scanner variability in Hounsfield unit measured by CT of the brain and effect on gray-towhite matter ratio. Am J Emerg Med 37:680-684

393. Lee BK, Jeung KW, Song KH et al (2015) Prognostic values of gray matter to white matter ratios on early brain computed tomography in adult comatose patients after out-of-hospital cardiac arrest of cardiac etiology. Resuscitation 96:46-52

394. Moseby-Knappe M, Pellis T, Dragancea I et al (2017) Head computed tomography for prognostication of poor outcome in comatose patients after cardiac arrest and targeted temperature management. Resuscitation 119:89-94

395. Sandroni C, D’Arrigo S, Nolan JP (2018) Prognostication after cardiac arrest. Crit Care 22:150

396. Greer DM, Scripko PD, Wu O et al (2013) Hippocampal magnetic resonance imaging abnormalities in cardiac arrest are associated with poor outcome. J Stroke Cerebrovasc Dis 22:899-905

397. Jang J, Oh SH, Nam Y et al (2019) Prognostic value of phase information of 2D T2*-weighted gradient echo brain imaging in cardiac arrest survivors: a preliminary study. Resuscitation 140:142-149

398. Jeon CH, Park JS, Lee JH et al (2017) Comparison of brain computed tomography and diffusion-weighted magnetic resonance imaging to predict early neurologic outcome before target temperature management comatose cardiac arrest survivors. Resuscitation 118:21-26

399. Ryoo SM, Jeon SB, Sohn CH et al (2015) Predicting outcome with diffusion-weighted imaging in cardiac arrest patients receiving hypothermia therapy: multicenter retrospective cohort study. Crit Care Med 43:2370-2377

400. Kim J, Kim K, Hong S et al (2013) Low apparent diffusion coefficient cluster-based analysis of diffusion-weighted MRI for prognostication of out-of-hospital cardiac arrest survivors. Resuscitation 84:1393-1399

401. Moon HK, Jang J, Park KN et al (2018) Quantitative analysis of relative volume of low apparent diffusion coefficient value can predict neurologic outcome after cardiac arrest. Resuscitation 126:36-42

402. Hirsch KG, Fischbein N, Mlynash M et al (2020) Prognostic value of diffusion-weighted MRI for post-cardiac arrest coma. Neurology 94:e1684-e1692

403. Bongiovanni F, Romagnosi F, Barbella G et al (2020) Standardized EEG analysis to reduce the uncertainty of outcome prognostication after cardiac arrest. Intensive Care Med 46:963-972

404. Hirsch LJ, LaRoche SM, Gaspard N et al (2013) American Clinical Neurophysiology Society's Standardized Critical Care EEG Terminology: 2012 version. J Clin Neurophysiol 30:1-27

405. Sharshar T, Citerio G, Andrews PJ et al (2014) Neurological examination of critically ill patients: a pragmatic approach. Report of an ESICM expert panel. Intensive Care Med 40:484-495

406. Greer DM, Shemie SD, Lewis A et al (2020) Determination of brain death/death by neurologic criteria: the World Brain Death Project. JAMA 324:1078-1097

407. Sandroni C, Grippo A, Nolan JP (2020) ERC-ESICM guidelines for prognostication after cardiac arrest: time for an update. Intensive Care Med 46:1901-1903

408. Beuchat I, Solari D, Novy J, Oddo M, Rossetti AO (2018) Standardized EEG interpretation in patients after cardiac arrest: correlation with other prognostic predictors. Resuscitation 126:143-146
409. Fredland A, Backman S, Westhall E (2019) Stratifying comatose postanoxic patients for somatosensory evoked potentials using routine EEG. Resuscitation 143:17-21

410. Beuchat I, Novy J, Barbella G, Oddo M, Rossetti AO (2020) EEG patterns associated with present cortical SSEP after cardiac arrest. Acta Neurol Scand 142:181-185

411. Scarpino M, Lolli F, Lanzo G, et al. (2021) Does a combination of $>/=2$ abnormal tests vs. the ERC-ESICM stepwise algorithm improve prediction of poor neurological outcome after cardiac arrest? A post-hoc analysis of the ProNeCA multicentre study. Resuscitation 160:158-167

412. Bouwes A, Binnekade JM, Kuiper MA et al (2012) Prognosis of coma after therapeutic hypothermia: a prospective cohort study. Ann Neurol 71:206-212

413. Hakimi K, Kinney G, Kraft G, Micklesen P, Robinson L (2009) Reliability in interpretation of median somatosensory evoked potentials in the setting of coma: factors and implications. Neurocrit Care 11:353-361

414. Wijdicks EF, Hijdra A, Young GB, Bassetti CL, Wiebe S (2006) Practice parameter: prediction of outcome in comatose survivors after cardiopulmonary resuscitation (an evidence-based review): report of the Quality Standards Subcommittee of the American Academy of Neurology. Neurology 67:203-210

415. Gold B, Puertas L, Davis SP et al (2014) Awakening after cardiac arrest and post resuscitation hypothermia: are we pulling the plug too early? Resuscitation 85:211-214

416. Lybeck A, Cronberg T, Aneman A et al (2018) Time to awakening after cardiac arrest and the association with target temperature management. Resuscitation 126:166-171

417. Nakstad ER, Staer-Jensen H, Wimmer H et al (2020) Late awakening, prognostic factors and long-term outcome in out-of-hospital cardiac arrest-results of the prospective Norwegian Cardio-Respiratory Arrest Study (NORCAST). Resuscitation 149:170-179

418. Cronberg T, Kuiper M (2017) Withdrawal of life-sustaining therapy after cardiac arrest. Semin Neurol 37:81-87

419. Levin PD, Sprung CL (2005) Withdrawing and withholding life-sustaining therapies are not the same. Crit Care 9:230-232

420. Sprung CL, Woodcock T, Sjokvist P et al (2008) Reasons, considerations, difficulties and documentation of end-of-life decisions in European intensive care units: the ETHICUS Study. Intensive Care Med 34:271-277

421. Sprung CL, Ricou B, Hartog CS et al (2019) Changes in end-of-life practices in European Intensive Care Units From 1999 to 2016. JAMA 322:1-12

422. Elmer J, Torres C, Aufderheide TP et al (2016) Association of early withdrawal of life-sustaining therapy for perceived neurological prognosis with mortality after cardiac arrest. Resuscitation 102:127-135

423. May TL, Ruthazer R, Riker RR et al (2019) Early withdrawal of life support after resuscitation from cardiac arrest is common and may result in additional deaths. Resuscitation 139:308-313

424. Cochrane TI (2009) Unnecessary time pressure in refusal of life-sustaining therapies: fear of missing the opportunity to die. Am J Bioeth 9:47-54

425. Kitzinger J, Kitzinger C (2013) The "window of opportunity" for death after severe brain injury: family experiences. Sociol Health IIIn 35:1095-1112

426. Dale CM, Sinuff T, Morrison LJ, Golan E, Scales DC (2016) Understanding Early decisions to withdraw life-sustaining therapy in cardiac arrest survivors. a qualitative investigation. Ann Am Thorac Soc 13:1115-1122

427. Lazaridis C (2019) Withdrawal of Life-sustaining treatments in perceived devastating brain injury: the key role of uncertainty. Neurocrit Care 30:33-41

428. Downar J, Delaney JW, Hawryluck L, Kenny L (2016) Guidelines for the withdrawal of life-sustaining measures. Intensive Care Med 42:1003-1017

429. Matthews EA, Magid-Bernstein J, Presciutti A et al (2017) Categorization of survival and death after cardiac arrest. Resuscitation 114:79-82

430. Kim YJ, Ahn S, Sohn CH et al (2016) Long-term neurological outcomes in patients after out-of-hospital cardiac arrest. Resuscitation 101:1-5

431. Petzinka VN, Endisch C, Streitberger KJ et al (2018) Unresponsive wakefulness or coma after cardiac arrest-a long-term follow-up study. Resuscitation 131:121-127 
432. Phelps R, Dumas F, Maynard C, Silver J, Rea T (2013) Cerebral performance category and long-term prognosis following out-of-hospital cardiac arrest. Crit Care Med 41:1252-1257

433. Dyson K, Brown SP, May S et al (2019) International variation in survival after out-of-hospital cardiac arrest: a validation study of the Utstein template. Resuscitation 138:168-181

434. Smith $K$, Andrew E, Lijovic M, Nehme Z, Bernard S (2015) Quality of life and functional outcomes 12 months after out-of-hospital cardiac arrest. Circulation 131:174-181

435. Green CR, Botha JA, Tiruvoipati R (2015) Cognitive function, quality of life and mental health in survivors of our-of-hospital cardiac arrest: a review. Anaesth Intensive Care 43:568-576

436. Wilder Schaaf KP, Artman LK, Peberdy MA et al (2013) Anxiety, depression, and PTSD following cardiac arrest: a systematic review of the literature. Resuscitation 84:873-877

437. Sawyer KN (2020) Reintegration and recovery after surviving cardiac arrest: learning from the VACAR registry. Resuscitation 146:255-257

438. Tiainen M, Poutiainen E, Oksanen T et al (2015) Functional outcome, cognition and quality of life after out-of-hospital cardiac arrest and therapeutic hypothermia: data from a randomized controlled trial. Scand J Trauma Resusc Emerg Med 23:12

439. Caro-Codon J, Rey JR, Lopez-de-Sa E et al (2018) Long-term neurological outcomes in out-of-hospital cardiac arrest patients treated with targeted-temperature management. Resuscitation 133:33-39

440. Sabedra AR, Kristan J, Raina K et al (2015) Neurocognitive outcomes following successful resuscitation from cardiac arrest. Resuscitation 90:67-72

441. Lim C, Verfaellie M, Schnyer D, Lafleche G, Alexander MP (2014) Recovery, long-term cognitive outcome and quality of life following out-of-hospital cardiac arrest. J Rehabil Med 46:691-697

442. Orbo M, Aslaksen PM, Larsby K, Schafer C, Tande PM, Anke A (2016) Alterations in cognitive outcome between 3 and 12 months in survivors of out-of-hospital cardiac arrest. Resuscitation 105:92-99

443. Steinbusch CVM, van Heugten CM, Rasquin SMC, Verbunt JA, Moulaert VRM (2017) Cognitive impairments and subjective cognitive complaints after survival of cardiac arrest: a prospective longitudinal cohort study. Resuscitation 120:132-137

444. Moulaert VRM, van Heugten CM, Gorgels TPM, Wade DT, Verbunt JA (2017) Long-term Outcome after survival of a cardiac arrest: a prospective longitudinal cohort study. Neurorehabil Neural Repair 31:530-539

445. Lilja G, Nilsson G, Nielsen N et al (2015) Anxiety and depression among out-of-hospital cardiac arrest survivors. Resuscitation 97:68-75

446. Viktorisson A, Sunnerhagen KS, Johansson D, Herlitz J, Axelsson A (2019) One-year longitudinal study of psychological distress and selfassessed health in survivors of out-of-hospital cardiac arrest. BMJ Open 9:e029756

447. Presciutti A, Sobczak E, Sumner JA et al (2019) The impact of psychological distress on long-term recovery perceptions in survivors of cardiac arrest. J Crit Care 50:227-233

448. Rosman L, Ford J, Whited A et al (2016) Compound risk: History of traumatic stress predicts posttraumatic stress disorder symptoms and severity in sudden cardiac arrest survivors. Eur J Cardiovasc Nurs 15:372-379

449. Juan E, De Lucia M, Beaud V et al (2018) How do you feel? Subjective perception of recovery as a reliable surrogate of cognitive and functional outcome in cardiac arrest survivors. Crit Care Med 46:e286-e293

450. Lilja G, Nielsen N, Bro-Jeppesen J et al (2018) Return to work and participation in society after out-of-hospital cardiac arrest. Circ Cardiovasc Qual Outcomes 11:e003566

451. Geri G, Dumas F, Bonnetain F et al (2017) Predictors of long-term functional outcome and health-related quality of life after out-of-hospital cardiac arrest. Resuscitation 113:77-82

452. Bohm M, Lilja G, Finnbogadottir $\mathrm{H}$ et al (2019) Detailed analysis of health-related quality of life after out-of-hospital cardiac arrest. Resuscitation 135:197-204

453. Orbo M, Aslaksen PM, Larsby K et al (2015) Relevance of cognition to health-related quality of life in good-outcome survivors of out-ofhospital cardiac arrest. J Rehabil Med 47:860-866

454. Tiainen M, Vaahersalo J, Skrifvars MB, Hastbacka J, Gronlund J, Pettila $\checkmark$ (2018) Surviving out-of-hospital cardiac arrest: the neurological and functional outcome and health-related quality of life 1 year later. Resuscitation 129:19-23

455. Beesems SG, Wittebrood KM, de Haan RJ, Koster RW (2014) Cognitive function and quality of life after successful resuscitation from cardiac arrest. Resuscitation 85:1269-1274

456. Descatha A, Dumas F, Bougouin W, Cariou A, Geri G (2018) Work factors associated with return to work in out-of-hospital cardiac arrest survivors. Resuscitation 128:170-174

457. Kearney J, Dyson K, Andrew E, Bernard S, Smith K (2020) Factors associated with return to work among survivors of out-of-hospital cardiac arrest. Resuscitation 146:203-212

458. Kragholm K, Wissenberg M, Mortensen RN et al (2015) Return to work in out-of-hospital cardiac arrest survivors: a Nationwide Register-based follow-up study. Circulation 131:1682-1690

459. Larsson IM, Wallin E, Rubertsson S, Kristofferzon ML (2014) Healthrelated quality of life improves during the first 6 months after cardiac arrest and hypothermia treatment. Resuscitation 85:215-220

460. Moulaert VR, Wachelder EM, Verbunt JA, Wade DT, van Heugten CM (2010) Determinants of quality of life in survivors of cardiac arrest. J Rehabil Med 42:553-558

461. Bunch TJ, White RD, Khan AH, Packer DL (2004) Impact of age on longterm survival and quality of life following out-of-hospital cardiac arrest. Crit Care Med 32:963-967

462. Viktorisson A, Sunnerhagen KS, Poder U, Herlitz J, Axelsson AB (2018) Well-being among survivors of out-of-hospital cardiac arrest: a crosssectional retrospective study in Sweden. BMJ Open 8:e021729

463. Nehme Z, Andrew E, Bernard S, Smith K (2019) Sex differences in the quality-of-life and functional outcome of cardiac arrest survivors. Resuscitation 137:21-28

464. Verberne D, Moulaert V, Verbunt J, van Heugten C (2018) Factors predicting quality of life and societal participation after survival of a cardiac arrest: A prognostic longitudinal cohort study. Resuscitation 123:51-57

465. Elliott VJ, Rodgers DL, Brett SJ (2011) Systematic review of quality of life and other patient-centred outcomes after cardiac arrest survival. Resuscitation 82:247-256

466. Haydon G, van der Riet P, Maguire J (2017) Survivors' quality of life after cardiopulmonary resuscitation: an integrative review of the literature. Scand J Caring Sci 31:6-26

467. Grasner JTH, Tjelmeland IBM, Wnent J, Masteron S, Lilja G, Bein B, Bottiger BW, Rosell-Ortiz F, Nolan JP, Bossaert L, Perkins GD (2021) European Resuscitation Council Guidelines 2021: Epidemiology of cardiac arrest in Europe. Resuscitation

468. Schweickert WD, Pohlman MC, Pohlman AS et al (2009) Early physical and occupational therapy in mechanically ventilated, critically ill patients: a randomised controlled trial. Lancet 373:1874-1882

469. Brummel NE, Girard TD, Ely EW et al (2014) Feasibility and safety of early combined cognitive and physical therapy for critically ill medical and surgical patients: the Activity and Cognitive Therapy in ICU (ACT-ICU) trial. Intensive Care Med 40:370-379

470. Boncyk CS, Rengel KF, Pandharipande PP, Hughes CG (2019) In the ICU delirium post cardiac arrest. Curr Opin Crit Care 25:218-225

471. Excellence NIfHaC (2009) Rehabilitation afrer critical illness in adults. Clinical Guideline 83. In: Editor (eds) Book Rehabilitation afrer critical illness in adults. Clinical Guideline 83. National Institute for Health and Care Excellence, City

472. Boyce LW, Goossens PH, Moulaert VR, Pound G, van Heugten CM (2019) Out-of-hospital cardiac arrest survivors need both cardiological and neurological rehabilitation! Curr Opin Crit Care 25:240-243

473. Cronberg T, Greer DM, Lilja G, Moulaert V, Swindell P, Rossetti AO (2020) Brain injury after cardiac arrest: from prognostication of comatose patients to rehabilitation. Lancet Neurol 19:611-622

474. Mion M, Al-Janabi F, Islam S et al (2020) Care after REsuscitation: implementation of the United Kingdom's first dedicated multidisciplinary follow-up program for survivors of out-of-hospital cardiac arrest. Ther Hypothermia Temp Manag 10:53-59

475. Moulaert VR, van Heugten CM, Winkens B et al (2015) Early neurologically-focused follow-up after cardiac arrest improves quality of life at 1 year: a randomised controlled trial. Int J Cardiol 193:8-16

476. Moulaert VR, Goossens M, Heijnders IL, Verbunt JA, Heugten CM (2016) Early neurologically focused follow-up after cardiac arrest is cost-effective: a trial-based economic evaluation. Resuscitation 106:30-36 
477. Moulaert VR, Verbunt JA, Bakx WG et al (2011) "Stand still ..., and move on", a new early intervention service for cardiac arrest survivors and their caregivers: rationale and description of the intervention. Clin Rehabil 25:867-879

478. Moulaert VR, van Haastregt JC, Wade DT, van Heugten CM, Verbunt JA (2014) "Stand still ..., and move on", an early neurologicallyfocused follow-up for cardiac arrest survivors and their caregivers: a process evaluation. BMC Health Serv Res 14:34

479. Lilja G (2017) Follow-Up of cardiac arrest survivors: why, how, and when? A practical approach. Semin Neurol 37:88-93

480. Boyce LW, Goossens PH (2017) Rehabilitation after cardiac arrest: integration of neurologic and cardiac rehabilitation. Semin Neurol 37:94-102

481. Blennow Nordstrom E, Lilja G, Arestedt K et al (2017) Validity of the IQCODE-CA: an informant questionnaire on cognitive decline modified for a cardiac arrest population. Resuscitation 118:8-14

482. van Heugten C, Rasquin S, Winkens I, Beusmans G, Verhey F (2007) Checklist for cognitive and emotional consequences following stroke (CLCE-24): development, usability and quality of the self-report version. Clin Neurol Neurosurg 109:257-262

483. Blennow Nordstrom E, Lilja G (2019) Assessment of neurocognitive function after cardiac arrest. Curr Opin Crit Care 25:234-239

484. Nasreddine ZS, Phillips NA, Bedirian V et al (2005) The montreal cognitive assessment, MoCA: a brief screening tool for mild cognitive impairment. J Am Geriatr Soc 53:695-699

485. Koller AC, Rittenberger JC, Repine MJ et al (2017) Comparison of three cognitive exams in cardiac arrest survivors. Resuscitation 116:98-104

486. Cicerone KD, Goldin Y, Ganci K et al (2019) Evidence-based cognitive rehabilitation: systematic review of the literature from 2009 through 2014. Arch Phys Med Rehabil 100:1515-1533

487. Snaith RP (2003) The hospital anxiety and depression scale. Health Qual Life Outcomes 1:29

488. Larsson IM, Wallin E, Rubertsson S, Kristoferzon ML (2013) Relatives' experiences during the next of kin's hospital stay after surviving cardiac arrest and therapeutic hypothermia. Eur J Cardiovasc Nurs 12:353-359

489. Brown JP, Clark AM, Dalal H, Welch K, Taylor RS (2011) Patient education in the management of coronary heart disease. Cochrane Database Syst Rev Issue 12, CD008895

490. Israelsson J, Lilja G, Bremer A, Stevenson-Agren J, Arestedt K (2016) Post cardiac arrest care and follow-up in Sweden-a national web-survey. BMC Nurs 15:1

491. Sawyer KN, Brown F, Christensen R, Damino C, Newman MM, Kurz MC (2016) Surviving sudden cardiac arrest: a pilot qualitative survey study of survivors. Ther Hypothermia Temp Manag 6:76-84

492. van Wijnen HG, Rasquin SM, van Heugten CM, Verbunt JA, Moulaert VR (2017) The impact of cardiac arrest on the long-term wellbeing and caregiver burden of family caregivers: a prospective cohort study. Clin Rehabil 31:1267-1275

493. Zimmerli M, Tisljar K, Balestra GM, Langewitz W, Marsch S, Hunziker S (2014) Prevalence and risk factors for post-traumatic stress disorder in relatives of out-of-hospital cardiac arrest patients. Resuscitation 85:801-808

494. Van't Wout Hofland J, Moulaert V, van Heugten C, Verbunt J (2018) Long-term quality of life of caregivers of cardiac arrest survivors and the impact of witnessing a cardiac event of a close relative. Resuscitation 128:198-203

495. Adiguzel E, Yasar E, Kesikburun S et al (2018) Are rehabilitation outcomes after severe anoxic brain injury different from severe traumatic brain injury? A matched case-control study. Int J Rehabil Res 41:47-51

496. Shah MK, Carayannopoulos AG, Burke DT, Al-Adawi S (2007) A comparison of functional outcomes in hypoxia and traumatic brain injury: a pilot study. J Neurol Sci 260:95-99

497. Fertl E, Vass K, Sterz F, Gabriel H, Auff E (2000) Neurological rehabilitation of severely disabled cardiac arrest survivors. Part I. Course of post-acute inpatient treatment. Resuscitation 47:231-239

498. Jolliffe L, Lannin NA, Cadilhac DA, Hoffmann T (2018) Systematic review of clinical practice guidelines to identify recommendations for rehabilitation after stroke and other acquired brain injuries. BMJ Open 8:e018791
499. Lee SY, Amatya B, Judson R et al (2019) Clinical practice guidelines for rehabilitation in traumatic brain injury: a critical appraisal. Brain Inj 33:1263-1271

500. Winstein CJ, Stein J, Arena R et al (2016) Guidelines for Adult stroke rehabilitation and recovery: a guideline for healthcare professionals from the American Heart Association/American Stroke Association. Stroke 47:e98-e169

501. Needham DM, Davidson J, Cohen H et al (2012) Improving long-term outcomes after discharge from intensive care unit: report from a stakeholders' conference. Crit Care Med 40:502-509

502. Mehlhorn J, Freytag A, Schmidt K et al (2014) Rehabilitation interventions for postintensive care syndrome: a systematic review. Crit Care Med 42:1263-1271

503. Walsh TS, Salisbury LG, Merriweather JL et al (2015) Increased hospitalbased physical rehabilitation and information provision after intensive care unit discharge: the RECOVER randomized clinical trial. JAMA Intern Med 175:901-910

504. Anderson L, Taylor RS (2014) Cardiac rehabilitation for people with heart disease: an overview of Cochrane systematic reviews. Cochrane Database Syst Rev Issue 12, CD011273

505. Piepoli MF, Corra U, Adamopoulos S et al (2014) Secondary prevention in the clinical management of patients with cardiovascular diseases. Core components, standards and outcome measures for referral and delivery: a policy statement from the cardiac rehabilitation section of the European Association for Cardiovascular Prevention \& Rehabilitation. Endorsed by the Committee for Practice Guidelines of the European Society of Cardiology. Eur J Prev Cardiol 21:664-681

506. Piepoli MF, Hoes AW, Agewall S et al (2016) 2016 European Guidelines on cardiovascular disease prevention in clinical practice: The Sixth Joint Task Force of the European Society of Cardiology and Other Societies on Cardiovascular Disease Prevention in Clinical Practice (constituted by representatives of 10 societies and by invited experts) developed with the special contribution of the European Association for Cardiovascular Prevention \& Rehabilitation (EACPR). Eur Heart J 37:2315-2381

507. Anderson L, Thompson DR, Oldridge $\mathrm{N}$ et al (2016) Exercise-based cardiac rehabilitation for coronary heart disease. Cochrane Database Syst Rev Issue 1, CD001800

508. Taylor RS, Dalal H, Jolly K, Moxham T, Zawada A (2010) Home-based versus centre-based cardiac rehabilitation. Cochrane Database Syst Rev Issue 1, CD007130

509. Bjarnason-Wehrens B, McGee H, Zwisler AD et al (2010) Cardiac rehabilitation in Europe: results from the European Cardiac Rehabilitation Inventory Survey. Eur J Cardiovasc Prev Rehabil 17:410-418

510. Kakos LS, Szabo AJ, Gunstad J et al (2010) Reduced executive functioning is associated with poorer outcome in cardiac rehabilitation. Prev Cardiol 13:100-103

511. Franklin BA (2010) Cognitive impairment: a new predictor of exercise trainability and outcomes in cardiac rehabilitation? Prev Cardiol 13:97-99

512. Larsen KK, Christensen B, Sondergaard J, Vestergaard M (2013) Depressive symptoms and risk of new cardiovascular events or death in patients with myocardial infarction: a population-based longitudinal study examining health behaviors and health care interventions. PLoS One 8:e74393

513. Wilson BA (2000) Compensating for cognitive deficits following brain injury. Neuropsychol Rev 10:233-243

514. Zedlitz AM, Rietveld TC, Geurts AC, Fasotti L (2012) Cognitive and graded activity training can alleviate persistent fatigue after stroke: a randomized, controlled trial. Stroke 43:1046-1051

515. Wylie GR, Flashman LA (2017) Understanding the interplay between mild traumatic brain injury and cognitive fatigue: models and treatments. Concussion 2:CNC50

516. Kim YJ, Rogers JC, Raina KD et al (2017) Solving fatigue-related problems with cardiac arrest survivors living in the community. Resuscitation 118:70-74

517. Kim YJ, Rogers JC, Raina KD et al (2016) An intervention for cardiac arrest survivors with chronic fatigue: a feasibility study with preliminary outcomes. Resuscitation 105:109-115

518. Dougherty CM, Thompson EA, Lewis FM (2005) Long-term outcomes of a telephone intervention after an ICD. Pacing Clin Electrophysiol 28:1157-1167 
519. Cowan MJ, Pike KC, Budzynski HK (2001) Psychosocial nursing therapy following sudden cardiac arrest: impact on 2-year survival. Nurs Res 50:68-76

520. Dougherty CM, Pyper GP, Frasz HA (2004) Description of a nursing intervention program after an implantable cardioverter defibrillator. Heart Lung 33:183-190

521. Bendorf A, Kelly PJ, Kerridge IH et al (2013) An international comparison of the effect of policy shifts to organ donation following cardiocirculatory death (DCD) on donation rates after brain death (DBD) and transplantation rates. PLoS One 8:e62010

522. Nolan JP, Ferrando P, Soar J et al (2016) Increasing survival after admission to UK critical care units following cardiopulmonary resuscitation. Crit Care 20:219

523. Thuong M, Ruiz A, Evrard P et al (2016) New classification of donation after circulatory death donors definitions and terminology. Transpl Int 29:749-759

524. Sandroni C, Adrie C, Cavallaro F et al (2010) Are patients brain-dead after successful resuscitation from cardiac arrest suitable as organ donors? A systematic review. Resuscitation 81:1609-1614

525. West S, Soar J, Callaway CW (2016) The viability of transplanting organs from donors who underwent cardiopulmonary resuscitation: a systematic review. Resuscitation 108:27-33

526. Minambres E, Rubio JJ, Coll E, Dominguez-Gil B (2018) Donation after circulatory death and its expansion in Spain. Curr Opin Organ Transplant 23:120-129

527. Stiles MK, Wilde AAM, Abrams DJ, et al. (2021) 2020 APHRS/HRS expert consensus statement on the investigation of decedents with sudden unexplained death and patients with sudden cardiac arrest, and of their families. Heart Rhythm 18(1):e1-e50

528. Ranthe MF, Winkel BG, Andersen EW et al (2013) Risk of cardiovascular disease in family members of young sudden cardiac death victims. Eur Heart J 34:503-511

529. Skinner JR (2013) Investigation following resuscitated cardiac arrest. Arch Dis Child 98:66-71

530. Skinner JR (2012) Investigating sudden unexpected death in the young: a chance to prevent further deaths. Resuscitation 83:1185-1186

531. Fellmann F, van El CG, Charron P et al (2019) European recommendations integrating genetic testing into multidisciplinary management of sudden cardiac death. Eur J Hum Genet 27:1763-1773

532. Sinha SS, Chen LM, Nallamothu BK (2014) Survival by the fittest: hospital-level variation in quality of resuscitation care. J Am Heart Assoc 3:e000768

533. Carr BG, Goyal M, Band RA et al (2009) A national analysis of the relationship between hospital factors and post-cardiac arrest mortality. Intensive Care Med 35:505-511

534. May TL, Lary CW, Riker RR et al (2019) Variability in functional outcome and treatment practices by treatment center after out-of-hospital cardiac arrest: analysis of International Cardiac Arrest Registry. Intensive Care Med 45:637-646

535. Matsuyama T, Kiyohara K, Kitamura T et al (2017) Hospital characteristics and favourable neurological outcome among patients with out-ofhospital cardiac arrest in Osaka, Japan. Resuscitation 110:146-153

536. Tagami T, Hirata K, Takeshige T et al (2012) Implementation of the fifth link of the chain of survival concept for out-of-hospital cardiac arrest. Circulation 126:589-597

537. Kragholm K, Malta Hansen C, Dupre ME et al (2017) Direct transport to a percutaneous cardiac intervention center and outcomes in patients with out-of-hospital cardiac arrest. Circ Cardiovasc Qual Outcomes 10:e003414

538. Spaite DW, Bobrow BJ, Stolz U et al (2014) Statewide regionalization of postarrest care for out-of-hospital cardiac arrest: association with survival and neurologic outcome. Ann Emerg Med 64:496-506
539. Couper K, Kimani PK, Gale CP et al (2018) Patient, health service factors and variation in mortality following resuscitated out-of-hospital cardiac arrest in acute coronary syndrome: analysis of the Myocardial Ischaemia National Audit Project. Resuscitation 124:49-57

540. Soholm H, Kjaergaard J, Bro-Jeppesen J et al (2015) Prognostic implications of level-of-care at tertiary heart centers compared with other hospitals after resuscitation from out-of-hospital cardiac arrest. Circ Cardiovasc Qual Outcomes 8:268-276

541. Elmer J, Callaway CW, Chang CH et al (2019) Long-term outcomes of out-of-hospital cardiac arrest care at regionalized centers. Ann Emerg Med 73:29-39

542. Elmer J, Rittenberger JC, Coppler PJ et al (2016) Long-term survival benefit from treatment at a specialty center after cardiac arrest. Resuscitation 108:48-53

543. Andrew E, Nehme Z, Wolfe R, Bernard S, Smith K (2017) Long-term survival following out-of-hospital cardiac arrest. Heart 103:1104-1110

544. Mumma BE, Diercks DB, Wilson MD, Holmes JF (2015) Association between treatment at an ST-segment elevation myocardial infarction center and neurologic recovery after out-of-hospital cardiac arrest. Am Heart J 170:516-523

545. Tranberg T, Lippert FK, Christensen EF et al (2017) Distance to invasive heart centre, performance of acute coronary angiography, and angioplasty and associated outcome in out-of-hospital cardiac arrest: a nationwide study. Eur Heart J 38:1645-1652

546. Cournoyer A, Notebaert E, de Montigny L et al (2018) Impact of the direct transfer to percutaneous coronary intervention-capable hospitals on survival to hospital discharge for patients with out-of-hospital cardiac arrest. Resuscitation 125:28-33

547. Lick CJ, Aufderheide TP, Niskanen RA et al (2011) Take Heart America: a comprehensive, community-wide, systems-based approach to the treatment of cardiac arrest. Crit Care Med 39:26-33

548. Stub D, Smith K, Bray JE, Bernard S, Duffy SJ, Kaye DM (2011) Hospital characteristics are associated with patient outcomes following out-ofhospital cardiac arrest. Heart 97:1489-1494

549. Chocron R, Bougouin W, Beganton F et al (2017) Are characteristics of hospitals associated with outcome after cardiac arrest? Insights from the Great Paris registry. Resuscitation 118:63-69

550. Lai CY, Lin FH, Chu H et al (2018) Survival factors of hospitalized out-ofhospital cardiac arrest patients in Taiwan: a retrospective study. PLoS One 13:e0191954

551. Soholm H, Wachtell K, Nielsen SL et al (2013) Tertiary centres have improved survival compared to other hospitals in the Copenhagen area after out-of-hospital cardiac arrest. Resuscitation 84:162-167

552. McKenzie N, Williams TA, Ho KM et al (2018) Direct transport to a PCIcapable hospital is associated with improved survival after adult out-ofhospital cardiac arrest of medical aetiology. Resuscitation 128:76-82

553. Brooks SC, Scales DC, Pinto R et al (2016) The postcardiac arrest consult team: impact on hospital care processes for out-of-hospital cardiac arrest patients. Crit Care Med 44:2037-2044

554. Seiner J, Polasek R, Lejsek J, Strycek M, Karasek J (2018) Cardiac arrest center-1-year experience of the Regional Hospital Liberec. Cor et Vasa 60:e234-e238

555. Harnod D, Ma MHM, Chang WH, Chang RE, Chang CH (2013) Mortality factors in out-of-hospital cardiac arrest patients: a nationwide population-based study in Taiwan. Int J Gerontol 7:216-220

556. Patterson T, Perkins GD, Joseph J et al (2017) A Randomised tRial of Expedited transfer to a cardiac arrest centre for non-ST elevation ventricular fibrillation out-of-hospital cardiac arrest: The ARREST pilot randomised trial. Resuscitation 115:185-191 